\title{
A damage to crack transition model accounting for stress triaxiality formulated in a hybrid non-local implicit discontinuous Galerkin - cohesive band model framework
}

\author{
J. Leclerc, L. Wu, V. D. Nguyen, L. Noels* \\ University of Liège - Department of Aerospace and Mechanical Engineering, Computational \& Multiscale Mechanics \\ of Materials, Allée de la Dćouverte 9, B-4000 Liège, Belgium
}

\begin{abstract}
SUMMARY
Modelling the entire ductile fracture process remains a challenge. On the one hand, continuous damage models succeed in capturing the initial diffuse damage stage but are not able to represent discontinuities or cracks. On the other hand, discontinuous methods, as the cohesive zones, which model the crack propagation behaviour, are suited to represent the localised damaging process. However, they are unable to represent diffuse damage. Moreover, most of the cohesive models do not capture triaxiality effect.

In this paper, the advantages of the two approaches are combined in a single damage to crack transition framework. In a small deformation setting, a non-local elastic damage model is associated with a cohesive model in a discontinuous Galerkin finite element framework. A cohesive band model is used to naturally introduce a triaxiality-dependent behaviour inside the cohesive law. Practically, a numerical thickness is introduced to recover a 3D-state, mandatory to incorporate the in-plane stretch effects. This thickness is evaluated to ensure the energy consistency of the method and is not a new numerical parameter. The traction-separation law is then built from the underlying damage model.
\end{abstract}

The method is numerically shown to capture the stress triaxiality effect on the crack initiation and propagation. Copyright (C) 2016 John Wiley \& Sons, Ltd.

Received ...

KEY WORDS: Damage; Fracture; Discontinuous Galerkin Method; cohesive band method; cohesive zone method; stress triaxiality

\section{INTRODUCTION}

Modelling the fracture of ductile materials with accuracy remains a challenging topic for scientists and engineers. Despite the large number of existing models, no solution is nowadays able to be totally predictive when the whole process is considered. This process starts most often with a diffuse damage evolution due to microscopic defects interaction, followed by a strain localisation stage

\footnotetext{
${ }^{*}$ Correspondence to: L. Noels, University of Liège, A\&M-CM3, B52 Quartier Polytech 1, Allée de la Dćouverte 9, B4000 Liège, Belgium, Phone: +32 436648 26, Fax: +32 436695 05. E-mail: 1.noels@ulg.ac.be 
and the appearance of a macroscopic crack. Currently, in a finite element context, the traditional numerical models used for ductile fracture can be divided into two approaches.

On the one hand, continuous damage models (or CDM) allow modelling a large range of degradation process. They represent the progressive deterioration of material properties with internal variables $[1,2$, e.g.] and can be implemented in a classical finite element (FE) framework. These models succeed in the description of the diffuse damage process. Nevertheless, the (local) form becomes ill-posed during the strain-softening regime since damage tends to localise in a one element-thick volume, resulting in mesh-dependency issues [3, 4]. Regularisation methods $[5,6,7,8,9$, e.g. $]$ as non-local forms or gradient enhanced models can be involved to restore solution uniqueness and avoid these problems. For instance, the implicit non-local method $[8,10]$ postulates that the damage diffusion is determined by a non-local variable, separately interpolated from the displacement field. This diffusion phenomenon is thus dependent on a characteristic length, representative of the interaction of micro-defects. Transient-gradient damage models [9], i.e. with variable characteristic length, were developed to lower spurious damage spreading due to unrealistic strain values arising in non-local formulations. Besides, the phase field approach was recently extensively developed $[11$, e.g.] in which crack surfaces are replaced by diffusive or regularised crack surfaces. The formulation thus shares strong similarities with the non-local damage approach. We refer to the recent comparisons between the two approaches provided in [12, 13].

On the other hand, discontinuous approaches, as in fracture mechanics, represent the material deterioration in the process zone in a discrete way. The material degradation is supposed to occur at the crack tip through crack propagation while the properties of the uncracked parts are assumed intact. One of the most popular method is the cohesive zone model (CZM) and was initiated by Dugdale and Barenblatt [14, 15]. This model describes the irreversible evolution of the opening traction forces in terms of crack opening, through its traction-separation law (TSL). The introduction of these discontinuities requires important modifications inside the FE discretisation. Cracks can propagate through the elements themselves by mesh enrichment (as for eXtended Finite Element Method (xFEM) [16, 17]) or by element enrichment (as in Embedded localisation method (EFEM) [18]), or through their boundaries thanks to interface elements [19]. Such elements can be inserted on the fly where the crack initiation criterion is reached $[20,21]$ in which case, the cohesive law is called "extrinsic" as it represents only the crack opening. However, this dynamic insertion decreases the scalability of the method for 3D simulations as the mesh topology is modified. When these elements are introduced before the beginning of the simulation, the "intrinsic" TSL should represent the pre-crack material response [22, 23, e.g.]. Unfortunately, their presence induces non-consistent elastic responses, mesh-dependency, and makes the method unconsistent and unpractical for nonlinear material behaviours and complex crack patterns [24]. To avoid these problems, a consistent and efficient way to insert a cohesive law is to use a Discontinuous Galerkin (DG) finite element discretisation $[25,26,27,28,29,30,31,32$, e.g.]. In this hybrid framework, the cohesive elements are inserted from the beginning without modifying the structure response because the pre-crack stage is modelled by the DG interface terms. By this way, it can naturally manage a large number of simultaneous crack initiation and propagation stages without mesh modification. Unfortunately, this method implies an increase of the system size and a fine mesh to accurately describe the crack patterns. However, this drawback is mitigated by the high scalability of the method. Furthermore, all the crack insertion techniques require a fine mesh to correctly describe the patterns. 
Nevertheless, both previously described approaches, fracture and damage mechanics, cannot separately represent the whole ductile failure process with accuracy. On the one hand, the CDM succeeds in capturing the preliminary damage diffusion stage followed by its localisation. However, they cannot described physical discontinuities or cracks. Spurious damage spreading arises due to these unrealistic strain values in the non-local formulation [9]. Moreover, important element distortions appear in the most damaged elements surrounding the real crack surface, resulting in numerical issues. Remeshing techniques are thus necessary [33] but computationally inefficient while removing those elements is inaccurate and unconsistent, due to the loss of mass and elastic energy. Another way to insert a discontinuity is to use a thick level set approach [34] in which the non-locality is introduced by the diffusion of damage over a given distance in front of the crack tip. On the other hand, the use of CZM alone is only valid for brittle or small-scale yielding materials. Indeed, all the material degradation is concentrated in the process zone of the crack tip, which is not the case for ductile or diffuse damage processes. All these reasons led to develop non-local damage models with a crack transition in order to take advantage of the complementarity of both approaches [35].

Numerous papers have shown theoretical equivalences between non-local models and cohesive zone methods, as long as the dissipated energy is conserved, which is physically based [36, 37, 38, 39, 35, 40, 41, e.g.]. Therefore, the principle of a non-local CDM/CZM transition is to describe the initial diffuse damage stage and its localisation by recourse to a non-local CDM. Then, crack initiation and propagation stages are modelled by a CZM. The TSL can be constructed by conserving the equivalence of dissipated energy increments with the pure non-local model [38]. To ensure energy consistency, this one has to dissipate the amount of energy not yet dissipated during the damage diffusion stage. This remaining quantity is determined by comparison for a mode I crack in a one-dimensional setting in terms of a reached value of damage [39], an effective stress one [35] or by a coupling with the local volume [40]. The transition criterion should be based on an effective stress rather than a critical damage value to avoid multiple crack initiation around an element of uniform damage [35]. However, as explained in [35], these methods do not account for triaxiality effects during the crack propagation stage: they remain accurate only when the crack is inserted near the complete failure at a damage close to 1 . In these cases, the shape of the cohesive law and the apparent Poisson effect (for some materials) are not of primary importance [42, 43]. Unfortunately, the physical crack initiation does not begin at such high damage values for all materials. The limitation results from the fact that classical CZM do not take into account the inplane stretch deformations while these strain components are key parameters in the ductile fracture process. Indeed, in-plane stretches, through triaxiality, pressure, or Lode variable effects, play an important role in the ductile failure $[44,45,46]$. To accurately describe this process, it has logically to be taken into account in the traction-separation law. Consequently, a general damage to crack transition model accounting for stress triaxiality remains to be developed.

The cohesive law can be modified to account for the stress triaxiality state: a simple way is to make the key parameters (critical stress and fracture energy) directly dependent on triaxiality [47]; the cohesive parameters can also be defined in terms of the effective plastic strain [48] or in terms of the porosity evolution [49] in the context of a Gurson model [50]. A more general method is to assume the presence of a thin band around the crack surface. This so-called cohesive band model can be inserted for instance via an intrinsic cohesive law $[42,51]$ or trough a weak discontinuity 
of finite width embedded in the elements $[52,53]$. Because of its 3D nature, the cohesive band naturally incorporates a triaxiality-dependent behaviour during the crack propagation stage. Besides, a coupling can be introduced between the irreversible evolution of a cohesive law and the damage process of an energetic interface $[54,55,56]$. The cohesive behaviour can also be extracted from microscale localisation in a multiscale analysis [57, 58].

The objective of this paper is to develop a computationally efficient and energetically consistent damage to crack transition framework for fracture analyses of elastic material, capturing triaxiality effects, and paving the way to more complex behaviours. To this end, an implicit non-local damage model and a cohesive band model (CBM) including a triaxiality-dependent behaviour are combined through the hybrid CDM/CBM framework. Concretely, the initial diffuse damage evolution is modelled by an implicit non-local damage model $[8,9,10]$. When the crack criterion is reached in the last failure steps, we assume that all the subsequent material degradation occurs in a thin band surrounding the crack surface. The cohesive model is taken as representative of this thin band behaviour. The strain state inside this band corresponds to the neighbouring bulk strains enriched with a cohesive jump. By redefining the underlying constitutive material model in a local form, a stress tensor, and then, the surface tension between the crack lips are evaluated.

This framework is developed in the following pages. Section 2 is dedicated to constitutive models. In particular, the formulation of the cohesive band model is detailed. Then, the energetic consistency is investigated in Section 3. In particular, the failure of a bar under tension is studied in a semianalytical way in order to derive a relation between the numerical thickness of the CBM and the characteristic length of the non-local damage model in such a way that the energy dissipated by the hybrid CDM/CBM framework corresponds to the energy that would be dissipated by a pure non-local damage simulation, i.e. without crack insertion. Therefore no new numerical parameter is introduced in the model. Using these results, Section 4 is devoted to the numerical implementation of the hybrid CDM/CBM framework in a parallel setting. The developed method is finally illustrated through numerical examples in Section 5. In particular, it is shown that the effects of triaxiality state can be captured by the cohesive band model. The mesh insensitivity of the CDM/CBM framework is first studied on a 2D holed plate. Then, by modifying the boundary conditions, different stress triaxiality states are investigated and the improvements brought by the new framework when compared to a pure continuum damage model and to a cohesive zone model, are demonstrated. The numerical predictions of the hybrid CDM/CBM on single edge notched specimens are then compared to the phase field results available in the literature [59]. Finally, the model is validated with experimental measurements from the literature [60] on the standard Compact Tension Specimen (CTS).

\section{CONSTITUTIVE MODELS FOR THE DAMAGE TO CRACK TRANSITION}

In this section the damage to crack transition framework is presented. At first, the non-local continuum damage mechanics model used for the early damage stage is detailed following a thermodynamical approach. Then, the formulation of the cohesive band model is developed. Finally, the relations are particularised for a linear isotropic elastic law involving a non-local damage model. 


\subsection{Non-local continuum damage mechanics}

Let $B_{0} \subset \mathcal{R}^{3}$ be a structural body in the reference configuration at initial time $t=0$, and $B$ be its counterpart in the current configuration at time $t>0$. Its boundary surface $\Gamma$ is divided into a Neumann part $\Gamma_{\mathrm{N}}$ where a surface traction $\bar{t}$ is prescribed and a Dirichlet part $\Gamma_{\mathrm{D}}$ where a displacement $\bar{u}$ is prescribed. These two distinct parts satisfy $\Gamma_{\mathrm{N}} \cap \Gamma_{\mathrm{D}}=\emptyset$ and $\Gamma_{\mathrm{N}} \cup \Gamma_{\mathrm{D}}=\Gamma$.

2.1.1. Kinematic relations During the time evolution of the body $B$, the motion of a material particle initially at the position $\boldsymbol{X} \subset B_{0}$ and currently at the position $\boldsymbol{x}(t) \subset B$, is defined by the mapping $\boldsymbol{x}(t)=\boldsymbol{\varphi}(\boldsymbol{X}, t)$. The displacement field is defined by $\boldsymbol{u}(t)=\boldsymbol{x}(t)-\boldsymbol{X}$. The derivative of the current spatial position in terms of the material configuration, or deformation gradient, is

$$
\mathbf{F}=\frac{\partial \varphi}{\partial \boldsymbol{X}}=\frac{\partial \boldsymbol{x}}{\partial \boldsymbol{X}}=\mathbf{I}+\frac{\partial \boldsymbol{u}}{\partial \boldsymbol{X}}
$$

and the determinant of this matrix $J=\operatorname{det}(\mathbf{F})$ is called Jacobian.

In a small deformation setting, the Cauchy strain tensor $\epsilon$ is derived from the displacement field $u$

$$
\boldsymbol{\epsilon}=\frac{1}{2}\left[\frac{\partial \boldsymbol{u}}{\partial \boldsymbol{X}}+\left(\frac{\partial \boldsymbol{u}}{\partial \boldsymbol{X}}\right)^{\mathrm{T}}\right]=\frac{1}{2}\left[\mathbf{F}+\mathbf{F}^{\mathrm{T}}\right]-\mathbf{I} .
$$

2.1.2. Balance relations The equilibrium equation over the volume $B$ of density $\rho$ reads, in presence of body-force $\boldsymbol{b}$ per unit mass,

$$
\rho \ddot{\boldsymbol{u}}=\nabla \cdot \boldsymbol{\sigma}+\rho \boldsymbol{b}
$$

with the following boundary conditions:

$$
\begin{array}{cc}
\boldsymbol{\sigma} \cdot \boldsymbol{n}=\overline{\boldsymbol{t}} & \text { on } \Gamma_{\mathrm{N}}, \\
\boldsymbol{u}=\overline{\boldsymbol{u}} & \text { on } \Gamma_{\mathrm{D}},
\end{array}
$$

where $\boldsymbol{n}$ is the outward unit normal to $\Gamma$ in the current configuration and $\boldsymbol{\sigma}$ is the Cauchy stress tensor.

2.1.3. Energetic relations The boundary value problem stated by Eqs. (3)-(4) is completed by a material constitutive law. In a small deformation setting, this law can be expressed as

$$
\boldsymbol{\sigma}(t)=\boldsymbol{\sigma}(\boldsymbol{\epsilon}(t) ; \boldsymbol{Z}(\tau), \tau \in[0, t])
$$

where $\boldsymbol{Z}(\tau)$ is the vector of internal variables representing all the material history. A thermodynamic potential function (so-called state function) can be associated to each equilibrium state of the system in terms the symmetric strain tensor $\boldsymbol{\epsilon}(t)$ and of the state variables $\boldsymbol{Z}(\tau)$. For simplicity, an elastic isothermal case in a small deformation setting with isotropic damage model is considered in this work. The (irreversible) material degradation is assumed to be described through a scalar damage variable $D$ which varies monotonically from 0 (undamaged material) to 1 , for complete fracture. At this point, the stiffness vanishes and the material is unable to sustain any subsequent load. 
If the free (reversible) Helmholtz energy per unit volume inside the damaged material, $\rho \psi$, is chosen as this thermodynamic potential, one has in terms of a damage variable and of a quadratic form of elastic strains:

$$
\rho \psi(\boldsymbol{\epsilon}, D)=\frac{1}{2} \boldsymbol{\epsilon}: \mathcal{C}^{\mathrm{D}}(D): \boldsymbol{\epsilon},
$$

where $\mathcal{C}^{\mathrm{D}}$ is the positive-definite fourth-order stiffness tensor in terms of the isotropic scalar damage value $D$. The evolution of this free energy with respect to time reads

$$
\rho \dot{\psi}(\boldsymbol{\epsilon}, D)=\rho \frac{\partial \psi}{\partial \boldsymbol{\epsilon}}: \dot{\boldsymbol{\epsilon}}+\rho \frac{\partial \psi}{\partial D} \dot{D}=\boldsymbol{\sigma}: \dot{\boldsymbol{\epsilon}}-Y \dot{D} \text { with }\left\{\begin{array}{l}
\boldsymbol{\sigma}=\rho \frac{\partial \psi}{\partial \boldsymbol{\epsilon}}=\mathcal{C}^{\mathrm{D}}: \boldsymbol{\epsilon} \\
Y=-\rho \frac{\partial \psi}{\partial D}=-\frac{1}{2} \boldsymbol{\epsilon}: \frac{\partial \mathcal{C}^{\mathrm{D}}}{\partial D}: \boldsymbol{\epsilon}
\end{array}\right.
$$

where $Y$ is defined as the damage energy release rate.

The variation of dissipated energy $\dot{\phi}$ is constrained by the second thermodynamic principle formulated by the Clausius-Duhem inequality

$$
\dot{\phi}=\dot{W}_{\text {int }}-\rho \dot{\psi}=\boldsymbol{\sigma}: \dot{\boldsymbol{\epsilon}}-\rho \dot{\psi} \geq 0,
$$

in which the first term involves the specific internal work $W_{\text {int }}$. By using the previous Eq. (7), one has

$$
\dot{\phi}=Y \dot{D} \geq 0
$$

which implies $\dot{D}, Y \geq 0$ and $\frac{\partial \mathcal{C}^{\mathrm{D}}}{\partial D}$ to be a semi-negative definite tensor matrix. The total dissipated power inside the structural volume $B$ is determined by

$$
\dot{\Phi}_{\mathrm{vol}}=\int_{B} \dot{\phi} d V=\int_{B} Y \dot{D} d V \geq 0
$$

The evolution of the damage $D$ is governed by the strain history of the material trough a loading function $f \leq 0$ that governs the damage growth. In its local form, the loading function depends only on local strain components and history by a function $\mathcal{F}$ through:

$$
\dot{D}= \begin{cases}\mathcal{F}(Y, \boldsymbol{Z}) & \text { if } f(D(t), \boldsymbol{\epsilon}(t) ; \boldsymbol{Z}(\tau), \tau \in[0, t])=0 \text { and } \dot{f}=0 \\ 0 & \text { if } f<0, \text { or } f=0 \text { and } \dot{f}<0\end{cases}
$$

However, this local form loses its solution uniqueness when softening appears, resulting in spurious localisation and mesh dependency issues. A well-posed problem can be recovered by using the non-local implicit method [10]. The main idea is to replace one internal variable $\chi \in Z$ (as damage, accumulated plastic strains...) by its non-local counterpart $\tilde{\chi} \in \tilde{Z}$, which corresponds to a weighted average on neighbouring material points. In other words, $\tilde{\chi}$ can be computed by

$$
\tilde{\chi}(\mathbf{X})=\frac{1}{V_{0}} \int_{V_{0}} \chi(\mathbf{Y}) w(\|\mathbf{Y}-\mathbf{X}\|) d V
$$

where $V_{0}$ is a characteristic volume around point $\mathbf{X}$, and $w(\|\mathbf{Y}-\mathbf{X}\|)$ is the normalised weight function that depends on the distance between both points $\mathbf{X}$ and $\mathbf{Y}$ only. This function $w$ defines 
the influence of the neighbouring material points and satisfies

$$
\frac{1}{V_{0}} \int_{V_{0}} w(\|\mathbf{Y}-\mathbf{X}\|) d V=1
$$

As the integral form of Eq. (12) would be difficult to evaluate for non-regular geometries, it is therefore replaced by a more convenient diffusion-like partial differential equations. Indeed, as detailed in [3], for specific weight functions, Eq. (12) can be transformed into a differential form associated with a natural boundary condition:

$$
\begin{array}{rlrl}
\tilde{\chi}-l_{c}^{2} \triangle \tilde{\chi} & =\chi & & \text { on } B_{0}, \\
\boldsymbol{\nabla} \tilde{\chi} \cdot \boldsymbol{n}=0 & & \text { on } \Gamma .
\end{array}
$$

The local value acts as a source term and $l_{c}$, the characteristic length of the material, governs the spreading or the averaging volume $V_{0}$ of the non-local value.

Adding this boundary value problem to Eq. (3) allows thus restoring the solution uniqueness. Therefore the damage evolution is rewritten in its non-local form in terms of the averaged variable:

$$
\dot{D}= \begin{cases}\mathcal{F}(Y, \tilde{Z}, \boldsymbol{Z}) & \text { if } f(D(t), \boldsymbol{\epsilon}(t) ; \tilde{\boldsymbol{Z}}(\tau), \boldsymbol{Z}(\tau), \tau \in[0, t])=0 \text { and } \dot{f}=0 \\ 0 & \text { if } f<0, \text { or } f=0 \text { and } \dot{f}<0\end{cases}
$$

Equations (6) to (16) remain valid for any isothermal isotropic elastic behaviour. In this paper, we consider a linear isotropic elastic law enhanced with an implicit non-local isotropic damage evolution to complete the boundary value problem stated by Eqs. (3)-(4) and (14)-(15). The constitutive relations are detailed in Section 2.3. Despite the simplicity of the model, this choice is motivated by the large number of studies available in the literature. Moreover, this model was calibrated for short glass-fiber reinforced polymers [60] and allows us to validate the applications in regard to experimental results.

\subsection{Cohesive band model}

Let $\Gamma_{\mathrm{b}}$ be a discontinuity surface dividing the structural volume $B$ into two parts: $B^{+}$and $B^{-}$. The unit normal $\boldsymbol{n}_{\mathrm{b}}$ to $\Gamma_{\mathrm{b}}$ is defined oriented towards $B^{+}$as illustrated in Fig. 1, the unit tangential vectors $t_{\mathrm{b}}$ and $s_{\mathrm{b}}$ define with $\boldsymbol{n}_{\mathrm{b}}$ an orthonormal local basis at the interface. The vectors $N_{\mathrm{b}}, T_{\mathrm{b}}$ and $\boldsymbol{S}_{\mathrm{b}}$ are their counterparts in the reference configuration. In the following equations, we introduce two operators to link variable values $\bullet^{+}$and $\bullet^{-}$from both sides of $\Gamma_{\mathrm{b} 0}$ :

$$
\begin{aligned}
& \text { the jump operator: } \llbracket \bullet \rrbracket=\left[\bullet^{+}-\bullet^{-}\right], \quad \text { and } \\
& \text { the mean operator: }\langle\bullet\rangle=\frac{1}{2}\left[\bullet^{+}+\bullet^{-}\right] \text {. }
\end{aligned}
$$

Pioneered by Dugdale and Barenblatt [14, 15], the cohesive zone model describes the irreversible evolution of attraction forces $\boldsymbol{t}_{\mathrm{b}}$ between both separation planes in terms of the crack opening $\llbracket \boldsymbol{u} \rrbracket$. In this case, the dissipated energy during the crack opening process corresponds to the fracture energy per unit crack surface $G_{\mathrm{C}}$ :

$$
G_{\mathrm{C}}=\int_{0}^{\delta_{\mathrm{C}}} \boldsymbol{t}_{\mathrm{b}} \cdot d \llbracket \boldsymbol{u} \rrbracket
$$




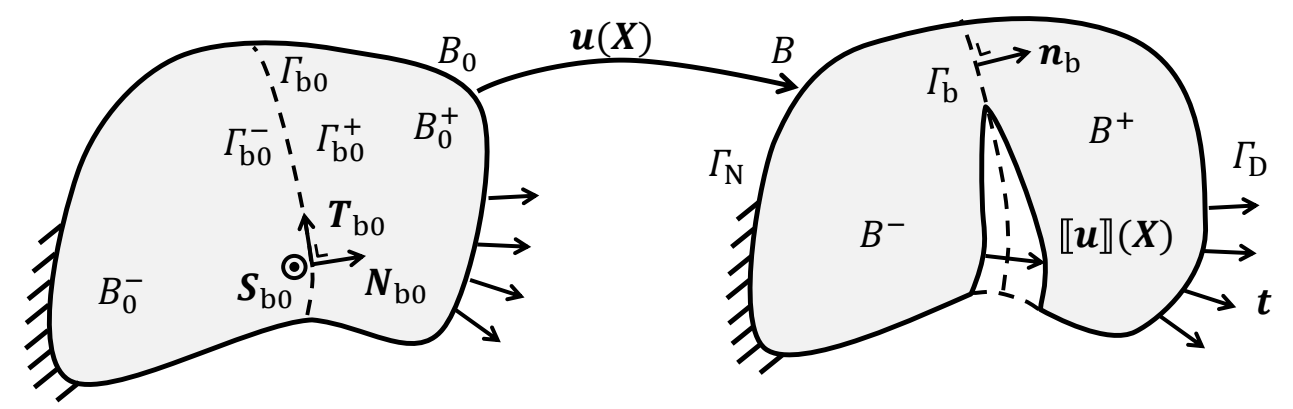

Figure 1. A discontinuity surface in the reference configuration $B_{0}$ (left) and the current configuration $B$ (right).

where $\delta_{c}$ is a critical opening value at which the complete fracture state is reached (i.e. when the attraction forces vanished). The dissipated power is related to the rate of crack surface creation $\dot{A}$ by

$$
\dot{\Phi}_{\text {surf }}=G_{\mathrm{C}} \dot{A}
$$

Hence, this model is intrinsically surfacic, by opposition to the bulk law which is volumic and depends on a 3D-state. In this paper, the idea exploited is to replace the classical cohesive zone model by a cohesive band one [42], representative of a thin band surrounding the crack surface. In this way, the underlying model is close to the reality of the failure process. Indeed, after a preliminary diffuse damage stage, all the degradation for ductile materials tends to localise in a thin band, before the appearance of macroscopic cracks and the complete loss of mechanical integrity. Moreover, this method reconstructs a pseudo 3D-state at the interface and harmonises itself with the volumic nature of the bulk law, thus including naturally triaxiality effects.

The displacement field $\boldsymbol{u}(\boldsymbol{X})$ inside the volume can be conveniently separated as illustrated in Fig. 2(a) into a smoothed (continuous) part $\boldsymbol{u}_{\mathrm{c}}(\boldsymbol{X})$ and a jump or discontinuous one $\llbracket \boldsymbol{u}(\boldsymbol{X}) \rrbracket$ according to:

$$
\boldsymbol{u}(\boldsymbol{X})=\boldsymbol{u}_{\mathrm{c}}(\boldsymbol{X})+\mathcal{H}_{\mathrm{D}}(\boldsymbol{X}) \llbracket \boldsymbol{u}(\boldsymbol{X}) \rrbracket,
$$

with the Heaviside's function $\mathcal{H}_{\mathrm{D}}(\boldsymbol{X})$

$$
\mathcal{H}_{\mathrm{D}}(\boldsymbol{X})= \begin{cases}0 & \text { if } \boldsymbol{X} \in B_{0}^{-} \\ 1 & \text { if } \boldsymbol{X} \in B_{0}^{+}\end{cases}
$$

By using the cohesive band model, the discontinuity surface is assumed to be smoothed and smeared through a numerical thin band $B_{\mathrm{b}}$ of thickness $h_{\mathrm{b}}$ as shown in Fig. 2(b). Without losing any generality, $\Gamma_{\mathrm{b}}$ is assumed to be locally planar and it corresponds to the mid-plan of the encompassing band $B_{\mathrm{b}}$. The displacement field $\boldsymbol{u}(\mathbf{X})$ is thus rewritten as

$$
\boldsymbol{u}(\boldsymbol{X})=\boldsymbol{u}_{\mathrm{c}}(\boldsymbol{X})+\mathcal{H}_{\mathrm{B}}(\boldsymbol{X}) \llbracket \boldsymbol{u}(\boldsymbol{X}) \rrbracket,
$$




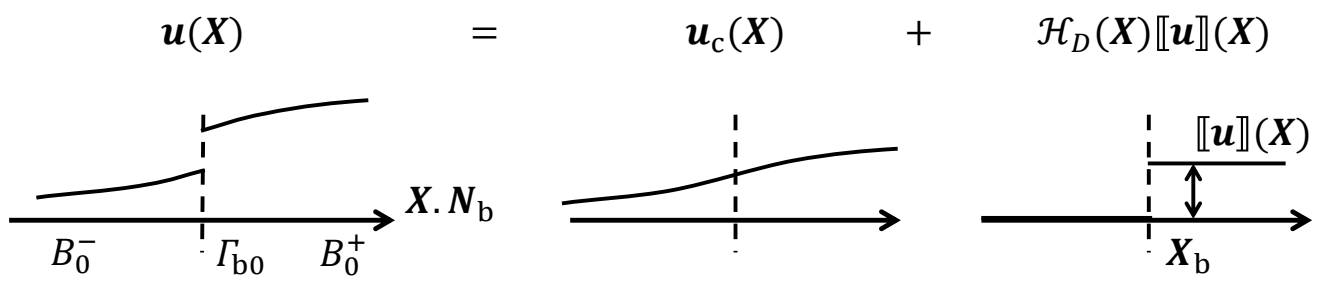

(a)
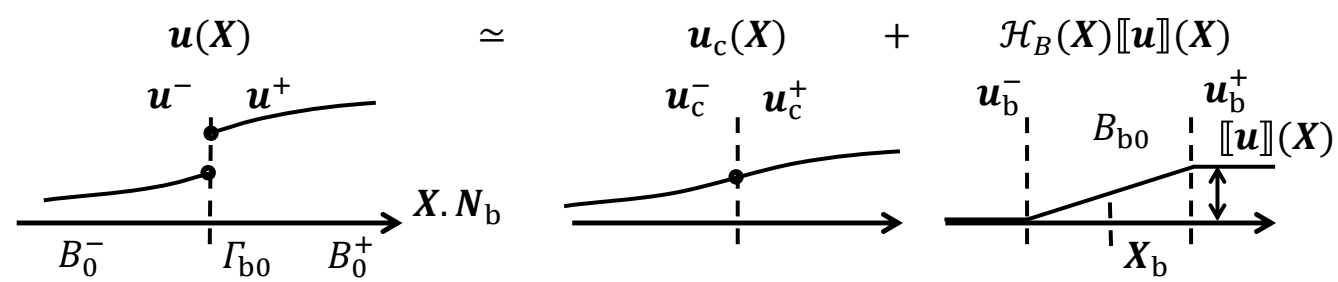

(b)

Figure 2. (a) Discontinuous displacement field decomposition around the crack surface $\Gamma_{\mathrm{b} 0}$ into a smoothed (continuous) part $\boldsymbol{u}_{\mathrm{c}}(\boldsymbol{X})$ and a jump or discontinuous one $\llbracket \boldsymbol{u}(\boldsymbol{X}) \rrbracket$. (b) With the cohesive band approximation, the discontinuous part is smeared through a numerical thin band $B_{\mathrm{b} 0}$. The displacement values at both crack sides, $u^{-}$and $u^{+}$, are obtained by respectively $u^{-}=u_{\mathrm{c}}^{-}+u_{\mathrm{b}}^{-}$and $u^{+}=u_{\mathrm{c}}^{+}+u_{\mathrm{b}}^{+}$.

with the function $\mathcal{H}_{\mathrm{B}}(\boldsymbol{X})$ corresponding to a piecewise linear-step:

$$
\mathcal{H}_{\mathrm{B}}(\boldsymbol{X})= \begin{cases}0 & \text { if } \boldsymbol{X} \in B_{0}^{-} \\ \frac{\left(\boldsymbol{X}-\boldsymbol{X}_{\mathrm{b}}\right) \cdot \boldsymbol{N}_{b}}{h_{\mathrm{b}}}+\frac{1}{2} & \text { if } \boldsymbol{X} \in B_{\mathrm{b} 0} \\ 1 & \text { if } \boldsymbol{X} \in B_{0}^{+}\end{cases}
$$

where $\boldsymbol{X}_{\mathrm{b}}$ is a point on the mid plan of the band, on the crack surface. By smearing the jump along the thickness, the displacement field remains continuous inside the band and allows us to define a strain tensor.

The band deformation gradient $\mathbf{F}_{\mathrm{b}}$ is computed by gerivating the band displacement field of Eq. (22), leading to

$$
\mathbf{F}_{\mathrm{b}}=\mathbf{I}+\frac{\partial \boldsymbol{u}_{\mathrm{c}}(\boldsymbol{X})}{\partial \boldsymbol{X}}+\frac{1}{h_{\mathrm{b}}} \llbracket \boldsymbol{u}(\boldsymbol{X}) \rrbracket \otimes \boldsymbol{N}_{\mathrm{b}}+\left(\frac{\left(\boldsymbol{X}-\boldsymbol{X}_{\mathrm{b}}\right) \cdot \boldsymbol{N}_{\mathrm{b}}}{h_{\mathrm{b}}}+\frac{1}{2}\right) \frac{\partial \llbracket \boldsymbol{u}(\boldsymbol{X}) \rrbracket}{\partial \boldsymbol{X}} .
$$

The band, resulting from strain localisation, is assumed to be very thin regarding to its longitudinal dimensions: the band thickness $h_{\mathrm{b}}$ is finite but negligible compared to others structural dimensions. The band deformation gradient $\mathbf{F}_{\mathrm{b}}$ deduced from Eq. (24) is thus approximated on both sides $\boldsymbol{X}_{\mathrm{b}}^{ \pm}$ accordingly with the chosen DG discretisation (see Section 4) by

$$
\mathbf{F}_{\mathrm{b}}^{ \pm}=\mathbf{F}^{ \pm}+\frac{1}{h_{\mathrm{b}}} \llbracket \boldsymbol{u} \rrbracket \otimes \boldsymbol{N}_{\mathrm{b}}+\frac{1}{2} \frac{\partial \llbracket \boldsymbol{u} \rrbracket}{\partial \boldsymbol{X}}
$$


where $\mathbf{F}^{ \pm}=\mathbf{F}\left(\boldsymbol{X}_{\mathrm{b}}^{ \pm}\right)$. In this last equation, the displacement discontinuity $\llbracket \boldsymbol{u}(\boldsymbol{X}) \rrbracket$ is assumed constant along the thickness, i.e

$$
\frac{\partial \llbracket \boldsymbol{u}(\boldsymbol{X}) \rrbracket}{\partial \boldsymbol{X}} \cdot \boldsymbol{N}=\mathbf{0},
$$

since the normal jump is of limited size with respect to the crack length.

For numerical stability reasons, the current jump-enhanced bulk components $\mathbf{F}_{\mathrm{N}}$, defined as

$$
\mathbf{F}_{\mathrm{N}}=\mathbf{F} \cdot \boldsymbol{N}_{\mathrm{b}} \otimes \boldsymbol{N}_{\mathrm{b}}
$$

are replaced by their counterparts at the crack insertion $\mathbf{F}_{\mathrm{Nc}}$, which are related to the deformation gradient at the crack insertion $\mathbf{F}_{\mathrm{c}}$ in the same way as Eq. (27). Indeed, using jump-enhanced current components implies a permanent stress equilibrium between the interface and the bulk which is not always the case during dynamic integration. If this balance is not satisfied, the delay in the decrease of the bulk contributions could induce spurious damage growth. In this paper $\mathbf{F}_{\mathrm{b}}^{ \pm}$is thus approximated by

$$
F_{\mathrm{b} i J}^{ \pm}=F_{i J}^{ \pm}+F_{\mathrm{Nc} i J}^{ \pm}-F_{\mathrm{N} i J}^{ \pm}+\frac{\llbracket u_{i} \rrbracket\left(N_{\mathrm{b}}\right)_{J}}{h_{\mathrm{b}}}+\frac{1}{2} \frac{\partial \llbracket u_{i} \rrbracket}{\partial X_{J}},
$$

or by using the local basis decomposition,

$$
\begin{aligned}
F_{\mathrm{b} i J}^{ \pm}=F_{i J}^{ \pm} & +\left(n_{\mathrm{b}}\right)_{i}\left(n_{\mathrm{b}}\right)_{k}\left[\left(F_{\mathrm{Nc} k L}^{ \pm}-I_{k L}\right) \frac{\delta_{\mathrm{n}}}{\delta_{\mathrm{n}}^{\text {max }}}-\left(F_{\mathrm{N} k L}^{ \pm}-I_{k L}\right)\right]\left(N_{\mathrm{b}}\right)_{L}\left(N_{\mathrm{b}}\right)_{J} \\
& +\left(t_{\mathrm{b}}\right)_{i}\left(t_{\mathrm{b}}\right)_{k}\left[\left(F_{\mathrm{Nc} k L}^{ \pm}-I_{k L}\right) \frac{\delta_{\mathrm{t}}}{\delta_{\mathrm{t}}^{\text {max }}}-\left(F_{\mathrm{N} k L}^{ \pm}-I_{k L}\right)\right]\left(N_{\mathrm{b}}\right)_{L}\left(N_{\mathrm{b}}\right)_{J} \\
& +\left(s_{\mathrm{b}}\right)_{i}\left(s_{\mathrm{b}}\right)_{k}\left[\left(F_{\mathrm{Nc} k L}^{ \pm}-I_{k L}\right) \frac{\delta_{\mathrm{s}}}{\delta_{\mathrm{s}}^{\text {max }}}-\left(F_{\mathrm{N} k L}^{ \pm}-I_{k L}\right)\right]\left(N_{\mathrm{b}}\right)_{L}\left(N_{\mathrm{b}}\right)_{J} \\
& +\frac{\llbracket u_{i} \rrbracket\left(N_{\mathrm{b}}\right)_{J}}{h_{\mathrm{b}}}+\frac{1}{2} \frac{\partial \llbracket u_{i} \rrbracket}{\partial X_{J}} .
\end{aligned}
$$

In this last equation, $\delta_{\mathrm{n}}, \delta_{\mathrm{t}}$, and $\delta_{\mathrm{s}}$ correspond to effective opening components along the directions of the local basis $\left(\boldsymbol{n}_{\mathrm{b}}, \boldsymbol{t}_{\mathrm{b}}, \boldsymbol{s}_{\mathrm{b}}\right)$ while $\delta_{\mathrm{n}}^{\max }, \delta_{\mathrm{t}}^{\max }$ and $\delta_{\mathrm{s}}^{\max }$ are their maximal values reached during crack opening. The introduction of the ratio coefficients $\delta_{\mathrm{n}} / \delta_{\mathrm{n}}^{\max }, \delta_{\mathrm{t}} / \delta_{\mathrm{t}}^{\max }$, and $\delta_{\mathrm{s}} / \delta_{\mathrm{s}}^{\max }$ allows recovering a deformation gradient equal to the identity in case of unloading up to crack closing.

In a similar way as to derive the bulk Cauchy strain tensor $\epsilon$ (2), its band counterpart $\epsilon_{\mathrm{b}}$ is derived from the band deformation gradient $\mathbf{F}_{\mathrm{b}}$ following

$$
\boldsymbol{\epsilon}_{\mathrm{b}}=\frac{1}{2}\left[\mathbf{F}_{\mathrm{b}}+\mathbf{F}_{\mathrm{b}}^{\mathrm{T}}\right]-\mathbf{I}
$$

Afterwards, the stress state $\sigma_{\mathrm{b}}$ inside the band is computed from the constitutive bulk law in its local version since the material in the band has a uniform internal variable state across its thickness. So, by similarity to Eq. (5), one has:

$$
\boldsymbol{\sigma}_{\mathrm{b}}(t)=\boldsymbol{\sigma}_{\mathrm{b}}\left(\boldsymbol{\epsilon}_{\mathrm{b}}(t) ; \boldsymbol{Z}(\tau), \tau \in[0, t]\right)
$$

where the damage evolution is described by a local damage model similar to Eq. (11):

$$
\dot{D}= \begin{cases}\mathcal{F}(Y, \boldsymbol{Z}) & \text { if } f\left(D(t), \boldsymbol{\epsilon}_{\mathrm{b}}(t) ; \boldsymbol{Z}(\tau), \tau \in[0, t]\right)=0 \text { and } \dot{f}=0 \\ 0 & \text { if } f<0, \text { or } f=0 \text { and } \dot{f}<0\end{cases}
$$


At the crack initiation occurring at time $t_{\mathrm{c}}$, for a deformation gradient $\mathbf{F}_{\mathrm{c}}$ and a Cauchy strain tensor $\epsilon_{\mathrm{c}}$, the damage and internal variables are initialised from the material state at cracking onset in order to avoid stress discontinuity and to conserve history continuity. The cohesive traction forces is now recovered by:

$$
\boldsymbol{t}_{\mathrm{b}}=\boldsymbol{\sigma}_{\mathrm{b}} \cdot \boldsymbol{n}_{\mathrm{b}} .
$$

By this way, the values of the cohesive forces are dependent on the in-plane stretch components and using the behaviour of that band as a cohesive law allows the triaxiality effects to be introduced during the last stage of failure within a formalism similar to a cohesive zone model.

\subsection{Implicit non-local damage model for isotropic elasticity}

All previous relations are applicable to elastic isothermal material laws with isotropic damage models in a small deformation setting and can be particularised for a specific material behaviour. We assume here linear isotropic elasticity modified to take into account the isotropic damaging process. By considering an isotropic damage model and a constant elastic tensor for linear elasticity, the free energy introduced in Eq. (6) becomes

$$
\rho \psi(\boldsymbol{\epsilon}, D)=\frac{1}{2}(1-D) \boldsymbol{\epsilon}: \mathcal{H}: \boldsymbol{\epsilon}
$$

where $\mathcal{C}^{\mathrm{D}}$ is replaced by $(1-D) \mathcal{H}_{i j k l}$, introducing $\mathcal{H}$ as the Hooke's tensor of the virgin material. The partial derivatives of the free energy yield the equations for the Cauchy stress tensor and the energy release rate

$$
\begin{aligned}
\boldsymbol{\sigma}=\rho \frac{\partial \psi}{\partial \boldsymbol{\epsilon}} & =(1-D) \mathcal{H}: \boldsymbol{\epsilon}=(1-D) \hat{\boldsymbol{\sigma}} \\
Y & =-\rho \frac{\partial \psi}{\partial D}=\frac{1}{2} \boldsymbol{\epsilon}: \mathcal{H}: \boldsymbol{\epsilon}
\end{aligned}
$$

by using the concept of effective Cauchy stress tensor $\hat{\sigma}$ introduced in [2]. These definitions are compatible with constraints resulting from Eq. (9) as long as $D$ is semi-monotonically increasing, considering the positive definite nature of the Hooke's tensor.

The evolution of the damage $D$, described by Eq. (16), is governed by the strain history of the material through an historical parameter $\kappa$. It corresponds to the maximum equivalent strain reached during the material history. The loading function in Eq. (16) reduces to

$$
f\left(\tilde{e}, D, \kappa_{\mathrm{i}}\right)=\tilde{e}-\kappa\left(\kappa_{\mathrm{i}}, D\right) \leq 0
$$

In this formula, $\tilde{e}$ is a non-local equivalent strain and $\kappa_{\mathrm{i}}$ is the initial threshold, limiting the domain of linear elasticity. The local equivalent strain $e$ is defined as the norm of the positive principal strain components $\epsilon_{i}^{+}$

$$
e=\sqrt{\sum_{i=1,2,3}\left(\epsilon_{i}^{+}\right)^{2}} .
$$

The non-local equivalent strain field is then obtained by solving the diffusion equation (14), which is rewritten as

$$
\tilde{e}-l_{\mathrm{c}}^{2} \triangle \tilde{e}=e,
$$


where $l_{\mathrm{c}}$ plays the role of the characteristic length of damage diffusion extension. The diffusion equation is completed with the natural boundary condition (15) which is rewritten as

$$
\nabla \tilde{e} \cdot \boldsymbol{n}=0
$$

In this paper, the damage law is a power-law, depending on two exponents: $\alpha$ and $\beta$. The damage evolution is triggered at $\kappa_{\mathrm{i}}$ and failure occurs when the strain reaches the critical value $\kappa_{\mathrm{c}}$ :

$$
\dot{D}(\kappa)= \begin{cases}0 & \text { if } \kappa<\kappa_{\mathrm{i}} ; \\ (1-D)\left(\frac{\beta}{\kappa}+\frac{\alpha}{\left(\kappa_{\mathrm{c}}-\kappa\right)}\right) \dot{\kappa} & \text { if } \kappa_{\mathrm{i}}<\kappa<\kappa_{\mathrm{c}} \\ 0 & \text { if } \kappa_{\mathrm{c}}<\kappa .\end{cases}
$$

By integration, an explicit function of $D$ in terms of $\kappa$ is available:

$$
D(\kappa)= \begin{cases}0 & \text { if } \kappa<\kappa_{\mathrm{i}} ; \\ 1-\left(\frac{\kappa_{i}}{\kappa}\right)^{\beta}\left(\frac{\kappa_{\mathrm{c}}-\kappa}{\kappa_{\mathrm{c}}-\kappa_{\mathrm{i}}}\right)^{\alpha} & \text { if } \kappa_{\mathrm{i}}<\kappa<\kappa_{\mathrm{c}} \\ 1 & \text { if } \kappa_{\mathrm{c}}<\kappa .\end{cases}
$$

After crack insertion, the damage evolution inside the band, described by the local form (32), is now depending on the local equivalent band strain. The damage growth criterion (36) becomes thus

$$
f\left(e_{\mathrm{b}}, D, \kappa_{\mathrm{i}}\right)=e_{\mathrm{b}}-\kappa\left(\kappa_{\mathrm{i}}, D\right) \leq 0,
$$

where $e_{\mathrm{b}}$ follows the variation of the local equivalent band strain $\dot{e}_{\mathrm{b}}=\dot{e}$, and is initiated at the crack insertion $e_{\mathrm{b}}\left(t_{\mathrm{c}}\right)=\tilde{e}\left(t_{\mathrm{c}}\right)$. Further details on the $\boldsymbol{\sigma}_{\mathrm{b}}$ computation are given in Appendix B.

\section{ENERGETIC EQUIVALENCE DURING TRANSITION}

In the damage to crack transition framework presented in the previous section, a new quantity was introduced: the cohesive band thickness. This variable is not an additional material parameter but this new numerical parameter can be determined from the underlying non-local model to ensure the energetic consistency. To this end, we study in this section the one-dimensional case in order to demonstrate the properties of the cohesive band model. In particular, the relation between the non-local length and the cohesive band thickness is derived to ensure energy consistency during the transition. The results can then be applied to general 3D simulations. Indeed, as the variations in the crack plane are negligible compared to those normal to the crack plane, the problem can be locally reduced to a one-dimensional problem at the crack front as suggested in [38].

\subsection{Energy consideration}

Several works have studied the equivalences between diffuse damage models and cohesive zone ones. In [38, e.g.], it has been shown that a cohesive zone can substitute for a non-local damage model as long as the total dissipated energy is conserved. 
Indeed, in a hybrid or coupled scheme, energy can be dissipated, on the one hand through damage or others internal variables evolution inside the volume elements $\left(d \Phi_{\mathrm{vol}}\right)$ following Eq. (10) or, on the other hand, through the crack opening of a cohesive zone $\left(d \Phi_{\text {surf }}\right)$ following Eq. (19). At the end of the process, the total dissipated energy $\Phi_{\text {tot,h }}$ is the sum of both contributions:

$$
\Phi_{\text {tot }, \mathrm{h}}=\Phi_{\mathrm{vol}}+\Phi_{\text {surf }}
$$

By comparison with a pure non-local model where all energy dissipation $\Phi_{\text {tot,nl }}$ results from the volume elements (i.e. $d \Phi_{\text {vol }} \neq 0, d \Phi_{\text {surf }}=0$ ), both frameworks (hybrid and pure non-local) are equivalent in an energetic point of view if the dissipated energy in both cases is conserved and corresponds to the physical fracture energy $\Phi_{\text {tot,phys. }}$ One has thus:

$$
\Phi_{\mathrm{tot}, \mathrm{phys}}=\Phi_{\mathrm{tot}, \mathrm{nl}}=\Phi_{\mathrm{tot}, \mathrm{h}}=\Phi_{\mathrm{vol}}+\Phi_{\text {surf }}
$$

This last equation can be rewritten as

$$
\Phi_{\text {surf }}=\Phi_{\text {tot }, \mathrm{nl}}-\Phi_{\mathrm{vol}}
$$

which gives an equation for $\Phi_{\text {surf }}$ and expresses the fact that the cohesive zone has to dissipate the remaining energy not yet dissipated by the non-local model to ensure an energetic equivalence between both frameworks.

\subsection{One dimensional problem setting}

Let us assume that the body $B$ reduces to a uniform bar of length $L$ and constant section $A$ with prescribed displacements at the extremities $\left(u(0, t)=0\right.$ and $\left.u(L, t)=\bar{u}_{\mathrm{L}}(t)\right)$. During the traction test assumed to occur under quasi-static conditions and without body forces, a strain localisation and finally a crack appears at $x_{0}=L / 2$, where $x$ is the axial coordinate varying in $[0, L]$.

3.2.1. Non-local problem Due to the geometry, the Cauchy stress tensor reduces to $\sigma_{x x}=\sigma$ with all the others components vanishing. The equilibrium equations (3) enforce a constant stress value $\sigma$ along the bar and the Hooke's law becomes

$$
\sigma=[1-D(\tilde{e}(x))] E \epsilon(x)
$$

where $E$ is the Young's modulus, and Eq. (2) is rewritten as

$$
\epsilon(x)=\frac{\partial u(x)}{\partial x} .
$$

This strain is linked to the boundary conditions by a compatibility equation:

$$
\int_{0}^{L} \epsilon(x) d x=\left.u\right|_{x=L}=\bar{u}_{\mathrm{L}}
$$


The non-local problem presented by Eqs. (38) and (39) reduces to

$$
\tilde{e}(x)-l_{\mathrm{c}}^{2} \frac{\partial^{2} \tilde{e}(x)}{\partial x^{2}}=e(x) \text { with }\left.\frac{\partial \tilde{e}(x)}{\partial x}\right|_{x=0, L}=0 .
$$

For a uni-axial traction, the equivalent strain $e(x)$ corresponds to $\epsilon(x)$.

This differential problem is equivalent to Eq. (12) with the Green functions $W(x, y)$ used as weigth functions, leading to

$$
\tilde{e}(x)=\int_{0}^{L} W(y, x) e(y) d y .
$$

For the one-dimensional bar problem, the corresponding Green functions [3] read

$$
W(x, y)=\frac{1}{2 l_{c}} \exp \left[-\frac{|x-y|}{l_{c}}\right]+W_{\mathrm{BC}}(x, y),
$$

with $W_{\mathrm{BC}}(x, y)$ depending on the boundary conditions

$$
W_{\mathrm{BC}}(x, y)=\frac{C_{1}(y)}{2 l_{c}} \exp \left[\frac{x}{l_{\mathrm{c}}}\right]+\frac{C_{2}(y)}{2 l_{\mathrm{c}}} \exp \left[\frac{L-x}{l_{\mathrm{c}}}\right] \text { where }\left\{\begin{array}{l}
C_{1}(y)=\frac{2 \cosh \left[\frac{y}{l_{\mathrm{c}}}\right]}{\exp \left[\frac{2 L}{l_{\mathrm{c}}}\right]-1} \\
C_{2}(y)=\frac{2 \cosh \left[\frac{L-y}{l_{\mathrm{c}}}\right]}{\exp \left[\frac{2 L}{l_{\mathrm{c}}}\right]-1} .
\end{array}\right.
$$

Finally, the damage evolution is computed from Eq. (41) with the damage growth criterion (36) and with $\kappa(t)=\max (\tilde{e}(\tau), \tau \in[0, t])$.

Using Eqs. (34), (46) and considering a uniform value of $\sigma$ along the bar, the increment of the internal work $d \mathcal{W}_{\text {int }}$ and the free energy $\Psi_{\text {vol }}$ stored in the system are written as

$$
\begin{gathered}
d \mathcal{W}_{\mathrm{int}}=A \int_{0}^{L} d W_{\mathrm{int}} d x=A \int_{0}^{L}(\sigma d \epsilon) d x=\sigma A d \bar{u}_{\mathrm{L}}, \\
\Psi_{\mathrm{vol}}=A \int_{0}^{L} \rho \psi d x=A \int_{0}^{L} \frac{1}{2} \sigma \epsilon(x) d x=\frac{A}{2} \sigma \bar{u}_{\mathrm{L}},
\end{gathered}
$$

and the dissipated energy inside the bar can be obtained as follows

$$
d \Phi_{\mathrm{vol}}=d \mathcal{W}_{\mathrm{int}}-d \Psi_{\mathrm{vol}}=A \sigma d \bar{u}_{\mathrm{L}}-\frac{A}{2} d\left(\sigma \bar{u}_{\mathrm{L}}\right)=\frac{A}{2}\left(\sigma d \bar{u}_{\mathrm{L}}-\bar{u}_{\mathrm{L}} d \sigma\right) .
$$

3.2.2. Cohesive band model When a crack is inserted, a discontinuity $\llbracket u \rrbracket$ in the displacement field is introduced at the centre of the localisation zone. The damaging process concentrates inside the cohesive zone while the remaining part of the bar is elastically unloaded. Thus the compatibility condition becomes:

$$
\bar{u}_{\mathrm{L}}=\int_{0}^{L} \epsilon(x) d x+\llbracket u \rrbracket .
$$

During this process, the damage is only increasing at the crack surface in a local way as expressed by Eq. (11) and depends on the band strain. The band strain is obtained by applying Eq. (29) in the 
case of a monotonically increasing jump:

$$
\epsilon_{\mathrm{b}}=\epsilon_{\mathrm{c}}\left(\frac{L}{2}\right)+\frac{\llbracket u \rrbracket}{h_{\mathrm{b}}},
$$

where $\epsilon_{\mathrm{c}}$ is the strain value at crack initiation. The bulk material is then elastically unloaded whilst the damage distribution in the volume remain constant and equal to the damage distribution $D_{\mathrm{c}}(x)$ reached at the insertion of the cohesive band. The equilibrium equation thus reads

$$
\sigma=\left(1-D_{\mathrm{c}}(x)\right) E \epsilon(x)=\sigma_{\mathrm{b}}=\left(1-D_{\mathrm{b}}\left(\epsilon_{\mathrm{b}}\right)\right) E \epsilon_{\mathrm{b}} .
$$

Following a similar approach than in [35], the free energy associated with the crack surface in a one-dimensional setting is assumed to be stated under the form $\Psi_{\text {surf }}=\Psi_{\text {surf }}(\llbracket u \rrbracket ; Z)$, or in other words, to be dependent on the jump and some internal variables $Z$. If the material unloading is linear elastic, the closing of the cohesive band follows the same non-dissipative behaviour. The free energy reduces thus to

$$
\Psi_{\text {surf }}=\frac{A}{2} \sigma \llbracket u \rrbracket,
$$

and, using the work done by the cohesive force $d \mathcal{W}_{\text {surf }}=A \sigma d \llbracket u \rrbracket$, the energy dissipation is equal to

$$
d \Phi_{\text {surf }}=d \mathcal{W}_{\text {surf }}-d \Psi_{\text {surf }}=A \sigma d \llbracket u \rrbracket-\frac{A}{2} d(\llbracket u \rrbracket \sigma)=\frac{A}{2}(\sigma d \llbracket u \rrbracket-\llbracket u \rrbracket d \sigma) .
$$

\subsection{Semi-analytical resolution of the localisation problem}

The equations for both cases (non-local and hybrid framework) have been simplified in the previous paragraphs to a one-dimensional case. Such a system of equations has been solved in [35] using a cohesive zone model (instead of a cohesive band model) by assuming that the transition occurs at a damage value close to one. In this paper, a more general solving strategy is developed in order to extract the cohesive band behaviour and to deduce an appropriate band thickness value.

3.3.1. Non-local problem The problem considers as unknowns the strain field, which is discretized and represented by its $n$ discrete values. From the discrete strain field $\epsilon^{i}, i=1, \ldots, n$, the non-local strain is computed by using the convolution of Eq. (50) and therefore the damage field and the stress field $\sigma^{i}$ are obtained from Eq. (41) and Eq. (46), respectively. As unstable branches can appear, the equations are formulated to involve an arc-length method [61, 62] to be able to capture snap-back behaviour. Concretely, an additional unknown, a loading parameter $\lambda$, is added, bringing the total number of unknowns to $n+1$. The system of $n+1$ equations can be rewritten under a residual form $\boldsymbol{r}\left(\epsilon^{i}, \lambda\right)$ as

$$
\boldsymbol{r}\left(\epsilon^{i}, \lambda\right)= \begin{cases}\sigma^{i+1}-\sigma^{i} & \text { for } i=1, \ldots, n-1, \\ \int_{0}^{L} \epsilon(x) d x-\lambda \bar{v} & \\ \sum_{j} \Delta \epsilon^{j} \Delta \epsilon^{j}+\vartheta^{2} \Delta \lambda^{2} \bar{v}^{2}-\Delta l^{2}, & \end{cases}
$$

where the first $n-1$ equations correspond to the static equilibrium, the next one to the compatibly equation, and the last one is introduced by the arc-length method to control the loading variation. In 
this last equation, $\vartheta$ is a numerical scaling parameter, $\bar{v}$ is a characteristic displacement $\bar{v}=\bar{u}_{\mathrm{L}} / \lambda$ which is associated with the loading parameter $\lambda$, and $\Delta l$ is the arc-length. In the last equation, $\Delta \epsilon^{j}$ and $\Delta \lambda$ correspond to the increments respectively of the $j^{\text {th }}$ discrete strain value and of the loading parameter between two consecutive steps. The integral in (60) is computed using the trapezoidal rule.

A predictor-corrector scheme with an iterative Newton-Raphson procedure is applied to solve the system, with the tangent matrix $\mathbf{K}_{\mathrm{al}}$ of the problem reading

$$
\mathbf{K}_{\mathrm{al}}=\left[\begin{array}{cc}
\frac{\partial \sigma^{i+1}}{\partial \epsilon^{j}}-\frac{\partial \sigma^{i}}{\partial \epsilon^{j}} & 0 \\
\frac{\partial}{\partial \epsilon^{j}} \int_{0}^{L} \epsilon(x) d x & -\bar{v} \\
2 \Delta \epsilon^{j} & 2 \vartheta^{2} \Delta \lambda \bar{v}^{2}
\end{array}\right]
$$

wherein the partial derivatives are computed by perturbation. As two possible solutions, namely elastic unloading or damage increase, always exist, the last one is promoted by the predictor that gives the guess state of the first iteration. Once the system has converged, the arc-length value $\Delta l$ is adapted in terms of the number of iterations needed to converge.

3.3.2. Crack insertion After crack insertion, the system is modified to take into account the discontinuity and the linear elastic unloading of the structure. As the bulk behaviour is now linear (linear elastic unloading), the problem reduces to only two unknowns: the total bulk displacement $\int_{0}^{L} \epsilon(x) d x$ and the crack opening $\llbracket u \rrbracket$. After transition, the jump, initially zero, is increased at each step and the corresponding cohesive band strain, damage, and stress are computed using Eqs. (56), (41) and (57), repectively. Knowing the axial stress, the bulk state can be deduced from its stiffness as it behaves linearly with respect to the stress.

\subsection{Band thickness computation and relation with the damage process zone}

As both cases (non-local and hybrid framework) can now be solved, we are able to compare both model dissipations, to characterise the behaviour of the cohesive band model, and to compute the energetically-consistent value of the band thickness. As a numerical example, the material properties are assimilated to short glass-fiber-reinforced polypropylene (short-GFRP) [60]. In this case, the Young's modulus $E$ has a value of $3.2 \mathrm{GPa}$. The damage law (40) parameters are $\kappa_{\mathrm{i}}=0.011, \kappa_{\mathrm{c}}=0.5, \alpha=5.0$, and $\beta=0.75$. Figure 3(a) shows the stress response in absence of strain localisation effect, or in other words, with a uniform strain value. By derivating Eq. (46), the damage value $D_{\text {soft }}$ at which strain softening regime begins is given by

$$
D_{\text {soft }}=D\left(\kappa_{\text {soft }}\right)=0.52 \text { with } \kappa_{\text {soft }}=\max \left(\frac{(1-\beta) \kappa_{\mathrm{c}}}{1-\beta+\alpha} ; \kappa_{i}\right)
$$

In order to trigger the localisation, a defect at the middle of the bar has been introduced in a similar way as in [63] by weakening by $1 \%$ the Young's modulus $E$ on $1 \%$ of the bar length (at the bar center). Figure 3(b) shows that the results are dependent on the ratio $l_{\mathrm{c}} / L$ : a lower value induces a more brittle behaviour. The fracture process can be divided in two parts. Before localisation, a homogeneous damage evolution takes place inside the bar, according to the curve on 3(a). Then, 


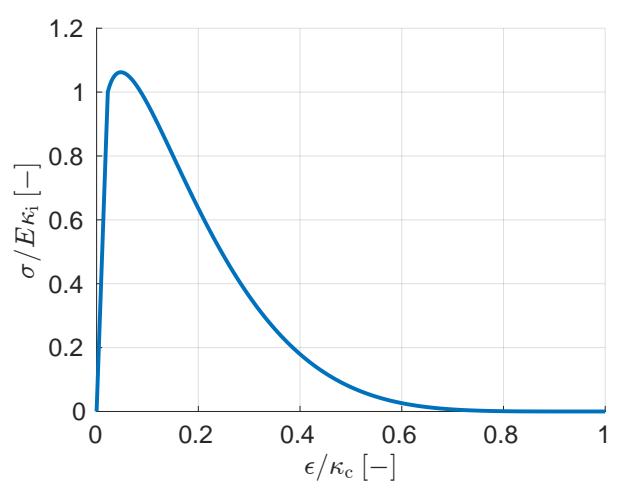

(a)

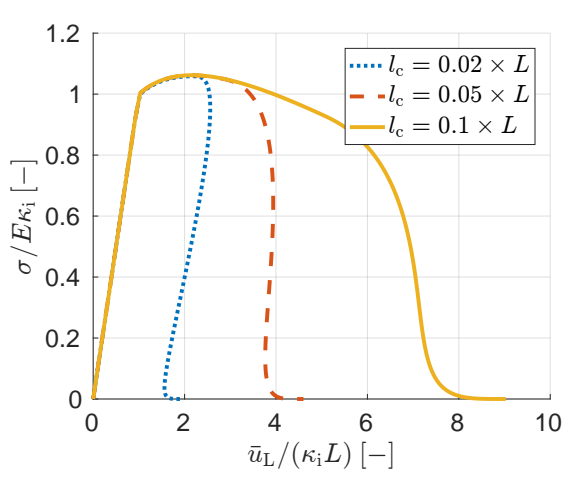

(b)

Figure 3. Stress response (a) for a uniform strain state, and (b) with localisation for different values of $l_{\mathrm{c}}$.

during the softening, a localisation zone develops around the defect, in which the damage grows, while other parts of the bar are elastically unloaded.

In the following analyses, the bar is taken of unitary length, $L=1 \mathrm{~m}$, and the non-local length is taken equal to $0.05 \mathrm{~L}$.

3.4.1. Influence of the band thickness $h_{b}$ Now, we introduce a discontinuity once a critical damage value $D_{\mathrm{c}}$ is reached. To have meaningful results, this value has to be high enough above $D_{\text {soft }}$ in order to be in the strain softening regime. Here, $D_{\mathrm{c}}$ is taken equal to 0.8 . At this point, $40 \%$ of the total fracture energy in the reference non-local case, $\Phi_{\text {tot,nl }}$, has already been dissipated (this value corresponds to $\Phi_{\text {vol }}$ in Eq. (43)) and a cohesive band is introduced. Figure 4(a) illustrates the effect of the band thickness on the stress response. By analogy to a classical cohesive zone, equivalent traction-separation laws can be extracted for comparison as shown in Fig. 4(b). One can see that a decreasing value of $h_{\mathrm{b}}$ leads to a more brittle behaviour of the bar. When $h_{\mathrm{b}}$ tends to 0 , the cohesive band is almost perfectly brittle. The corresponding dissipated energy $\Phi_{\text {toth }}$ is plotted in Fig. 5(a) and shows a linear dependency to $h_{\mathrm{b}}$. In particular, the contribution of the cohesive band to the dissipation vanishes for a vanishing band thickness. For this given critical damage value $\left(D_{\mathrm{c}}=0.8\right)$, the conservation of the total dissipated energy $\left(\Phi_{\text {tot, } \mathrm{h}}\left(h_{\mathrm{b}}\right)=\Phi_{\text {tot,nl }}\right)$ is obtained for $h_{\mathrm{b}}=5.4 l_{\mathrm{c}}$.

3.4.2. Influence of the non-local length on the band thickness As the non-local length is directly related to the spreading of damage, the dissipated energy increases linearly with $l_{\mathrm{c}}$ as long as there is no boundary effects, or in other words, as long as the damage process zone is much smaller than the bar length. The dissipated energy shown in Fig. 5(b) is conserved if the ratio $h_{\mathrm{b}} / l_{\mathrm{c}}$ is kept constant for small values of $l_{\mathrm{c}} / L$.

3.4.3. Influence of the critical damage on the band thickness The fracture energy which remains to be dissipated by a cohesive model $G_{\mathrm{c}}^{*}$, divided by the non-local characteristic length $l_{\mathrm{c}}$ to remove its influence, is represented in Fig. 6(a) in terms of the damage at crack insertion. The influence of the damage value at crack insertion on $h_{\mathrm{b}}$ is shown in Fig. 6(b). The lowest meaningful value of 


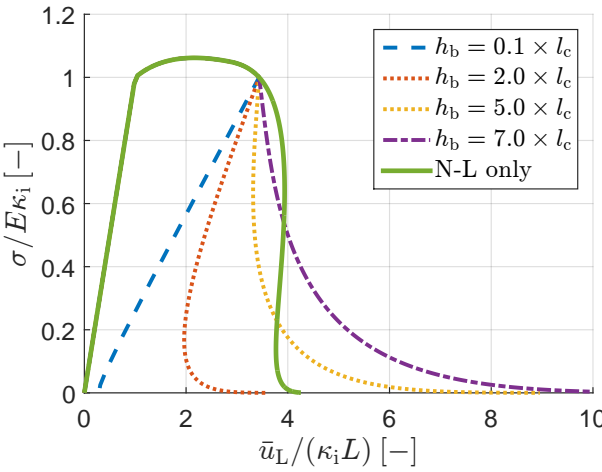

(a)

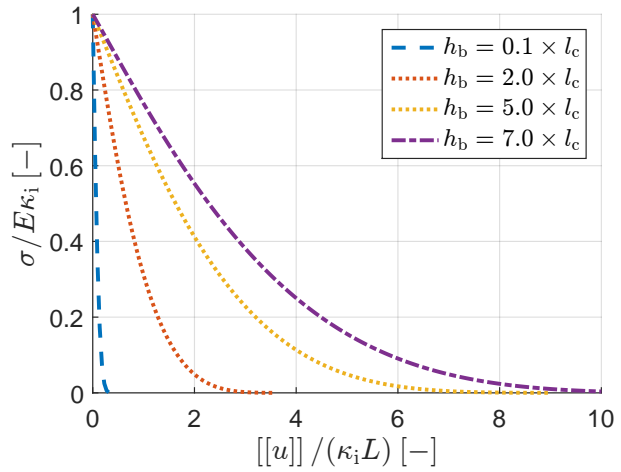

(b)

Figure 4. (a) Stress response for the hybrid framework with a cohesive band introduced at $D_{\mathrm{c}}=0.8$ and different values of $h_{\mathrm{b}}$. (b) The corresponding traction-separation laws.

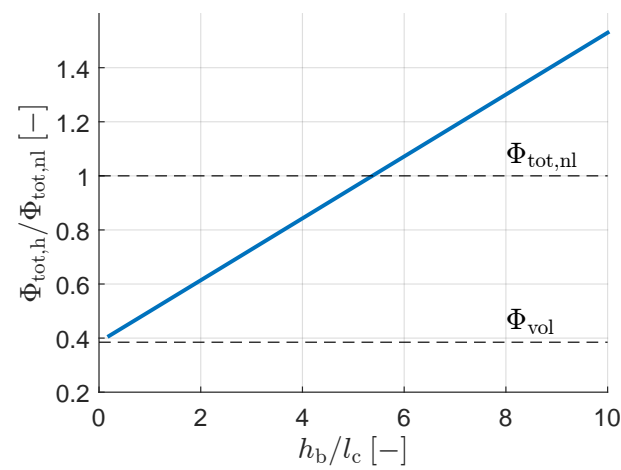

(a)

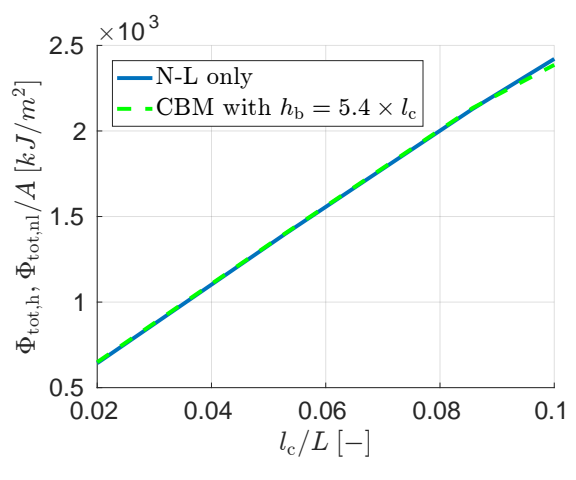

(b)

Figure 5. (a) The dissipated energy in terms of band thickness for $D_{\mathrm{c}}=0.8$ and $l_{\mathrm{c}}=0.05 L$ and (b) in terms of non-local length.

$D_{\text {c }}$ here is governed by the localisation onset, occurring practically at the uniform damage value observed far from the crack tip (here at $D=0.62$ ), which is slightly higher than the theorical (and minimal) value $D_{\text {soft }}=0.52$. This offset is due to the localisation latency and can be reduced if the ratio $l_{\mathrm{c}} / L$ is lowered. For moderate values of critical damage transition point, $h_{\mathrm{b}}$ is constant. Then, as $D_{\mathrm{c}}$ tends to one, the corresponding value of $h_{\mathrm{b}}$ starts growing rapidly. This unphysical behaviour can be explained by the involved non-local model: high strains values at the centre produces spurious damage diffusion and energy dissipation. This issue can be addressed by using a variable characteristic length as in [9].

3.4.4. Influence of the damage model on the band thickness In Figs. 7(a) and 7(b), the dissipated energy is represented in terms of respectively the damage exponent $\alpha$ and of the initial damage threshold $\kappa_{i}$ for the pure non-local model and for the CBM with $D_{\mathrm{c}}=0.8$. For the damage exponent 


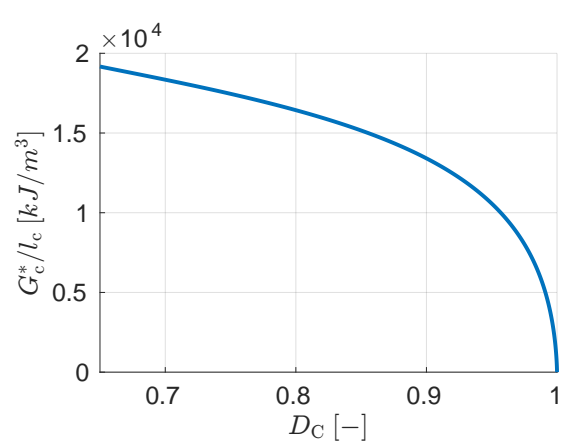

(a)

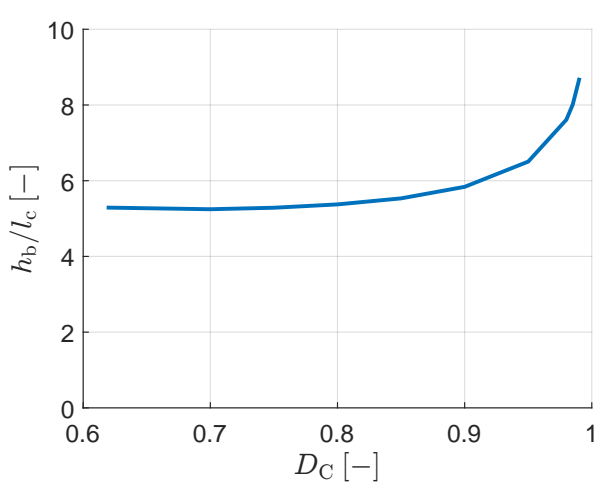

(b)

Figure 6. (a) The fracture energy which remains to be dissipated by the cohesive model $G_{\mathrm{c}}^{*}$ in terms of damage value of crack insertion. (b) The band thickness in terms of damage value of crack insertion.

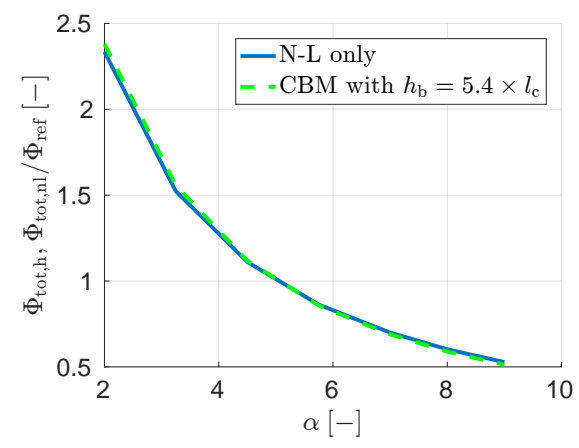

(a)

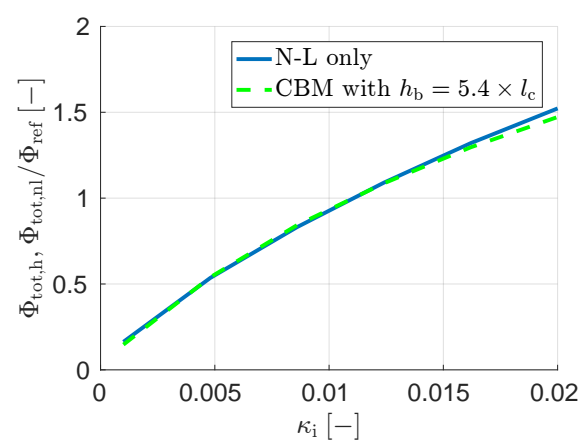

(b)

Figure 7. The dissipated energy for non-local model (a) in terms of the damage exponent $\alpha$ and (b) in terms of the initial damage threshold $\kappa_{i}$.

$\alpha$, the error in the energy due to the insertion of the CBM is within $2 \%$ for $\alpha \in[3.5 ; 8]$. This range of $\alpha$ ensures that localisation has occured at the crack insertion. For lower values of $\alpha$ crack insertion occurs before localisation regime, leading to a higher error on the dissipated energy. For the second parameter, the relative error on the dissipated energy is under $3 \%$ for $\kappa_{i} \in[0.005 ; 0.018]$. As $D_{\text {soft }}$ increases with smaller $\kappa_{i}$, a higher error is expected for such values $\left(9.6 \%\right.$ for $\kappa_{i}=0.001$ for which the corresponding $D_{\text {soft }}$ is equal to 0.927 ). Therefore, as long as the CBM is inserted during the localisation regime, the band thickness is independent of the damage model parameters as those effects are already included in the computation of the cohesive response by reusing the same constitutive relations.

3.4.5. Determination of the cohesive band thickness summary In this subsection we have studied the effect of the cohesive band thickness of the hybrid CDM/CBM framework on the failure of a 
one-dimensional bar under tension. It has been shown in Fig. 4 that increasing the numerical band thickness $h_{\mathrm{b}}$ results in increasing the ductility of the material since the energy dissipated by the model increases, see Fig. 5(a). However in order to avoid, on the one hand, the introduction of a new parameter, and, on the other hand, a method that would be sensitive to the point at which the damage to crack transition occurs, we have ensured energy consistency of the hybrid CDM/CBM with a pure non-local model, i.e. without crack insertion. Indeed, it has been shown that for the damage law (40), by selecting $h_{\mathrm{b}}=5.4 l_{\mathrm{c}}$, the hybrid CDM/CBM framework dissipates the same amount of energy than the pure non-local model for different values of the non-local length $l_{\mathrm{c}}$, for different points of the crack insertion, and for different possible parameters of the damage law. The numerical band thickness $h_{\mathrm{b}}$ is thus directly related to the non-local length $l_{\mathrm{c}}$ and is no longer a model parameter. However, the non-local length $l_{\mathrm{c}}$ remains a model parameter that affects the softening response. Moreover, if another damage model is used, the numerical band thickness $h_{\mathrm{b}}$ should be evaluated again in order to ensure energy consistency.

\section{DISCONTINUOUS GARLERKIN FRAMEWORK AND FINITE ELEMENT DISCRETISATION}

In this section, the implementation of the hybrid energetically-consistent CDM/CBM scheme inside a Discontinuous Galerkin (DG) framework is detailed. In particular, the weak form is developed from the strong form, leading to interface terms related to the DG formalism. Then, based on a finite element discretisation, a differential set of equations is obtained and integrated with an implicit or a coupled implicit-explicit scheme. The numerical properties of this scheme are also summarised.

\subsection{Discontinuous Galerkin framework}

The discontinuous Galerkin method is designed to solve various kinds of partial differential equations. Similarly to classical continuous Galerkin finite element methods, the geometry is also approached by polyhedral elements. Inside them, the interpolated field is approximated by nodal shape polynomial functions associated to each node. However, the support of a node is limited to the element to which it belongs. While the intra-element continuity is warranted by the shape functions, the inter-element continuity is therefore not strongly, but weakly, ensured by consistency and penalty terms introduced by the DG formalism. The inherent presence of interface discontinuities allows then naturally initiating and propagating a large number of cracks simultaneously without numerical problems by just switching the DG terms by the cohesive law. Despite the increase of the system size due to the formalism, the high scalability of the method mitigates this drawback. Indeed, the decoupling between elements eases parallel implementation. The following lines explain how the non-local DG formalism developed in [35] is extended to the hybrid CDM/CBM scheme.

4.1.1. Strong form of equations The evolution of the body $B$, determined by the field $(\boldsymbol{u}, \tilde{e})$ through time, is assessed by the set of partial differential equations (3) and (38) associated with boundary conditions (4) and (39). 
Cracked $\Gamma_{\mathrm{CI}}$ and uncracked $\Gamma_{\mathrm{UI}}$ interface surfaces are present inside the structural volume. As the exact solution $\left(\boldsymbol{u}^{\text {exact }}, e^{\text {exact }}, \boldsymbol{\sigma}^{\text {exact }}, \boldsymbol{\nabla} \tilde{e}^{\text {exact }}\right)$ is continuous on the uncracked surfaces, one has

$$
\begin{aligned}
& \llbracket \boldsymbol{u}^{\text {exact }} \rrbracket=\mathbf{0}, \quad \llbracket \boldsymbol{\sigma}^{\text {exact }} \rrbracket=\mathbf{0}, \\
& \llbracket \tilde{e}^{\text {exact }} \rrbracket=0, \quad \llbracket l_{\mathrm{c}}^{2} \boldsymbol{\nabla} \tilde{e}^{\text {exact }} \rrbracket=\mathbf{0} \quad \text { on } \Gamma_{\mathrm{UI}} .
\end{aligned}
$$

For the cracked parts, the interface traction $t_{\mathrm{b}}$ between both crack lips is determined by

$$
\llbracket \boldsymbol{t}_{\mathrm{b}} \rrbracket=\mathbf{0} ; \boldsymbol{t}_{\mathrm{b}}=\left\langle\boldsymbol{\sigma}_{\mathrm{b}}\right\rangle \cdot \boldsymbol{n}_{\mathrm{b}} ; \quad\left(l_{\mathrm{c}}^{2} \boldsymbol{\nabla} \tilde{e}^{\text {exact }}\right) \cdot \boldsymbol{n}_{\mathrm{b}}=0 \text { on } \Gamma_{\mathrm{CI}},
$$

which express that the traction forces are computed from the cohesive band model (33). The last equation of Eq. (64) considers a cracked surface as a free boundary in terms of the non-local variable, see a discussion in [35].

4.1.2. Weak form of equations The body $B$ of external surface $\Gamma$ is divided in finite elements $B^{e}$ of boundary $\Gamma^{e}$. The internal boundary of the elements $\Gamma_{\mathrm{I}}=\cup_{e} \Gamma^{e} \backslash \Gamma$ is divided between cracked interface surfaces $\Gamma_{\mathrm{CI}}$ and uncracked ones $\Gamma_{\mathrm{UI}}$, satisfying $\Gamma_{\mathrm{CI}} \cup \Gamma_{\mathrm{UI}}=\Gamma_{\mathrm{I}}$ and $\Gamma_{\mathrm{CI}} \cap \Gamma_{\mathrm{UI}}=\emptyset$. Between each pair of neighbouring elements (arbitrary called $B^{e+}$ and $B^{e-}$ ), an interface element $\Gamma^{s}$ is inserted on their common boundary surface $\Gamma^{s}=\Gamma^{e+} \cap \Gamma^{e-}$.

In a discontinuous Galerkin approach, an element-wise continuous polynomial approximation of $(\boldsymbol{u}, \tilde{e})$ is sought. Consequently, the trial functions $\left(\boldsymbol{w}_{\boldsymbol{u}}, \boldsymbol{w}_{\tilde{e}}\right)$ are also continuous inside the elements and discontinuous across them, i.e. $\left(\boldsymbol{w}_{\boldsymbol{u}}, \boldsymbol{w}_{\tilde{e}}\right) \in C^{0}\left(B^{e}\right)$ but $\left(\boldsymbol{w}_{\boldsymbol{u}}, \boldsymbol{w}_{\tilde{e}}\right) \notin C^{0}(B)$. The weak form is obtained by multiplying the differential equations by the trial functions and by performing an integration bt parts over each element. Indeed, as discontinuities are present inside the body, the integration has to be performed element-wise, resulting in the emergence of supplementary terms, so-called consistency terms, on the interfaces in comparison with classical continuous Galerkin methods. One thus has [35]

$$
\begin{array}{r}
\int_{B}\left(\rho \boldsymbol{w}_{\boldsymbol{u}} \cdot \ddot{\boldsymbol{u}}+\boldsymbol{\nabla} \boldsymbol{w}_{\boldsymbol{u}}: \boldsymbol{\sigma}\right) d V+\int_{\Gamma_{\mathrm{CI}}} \llbracket \boldsymbol{w}_{\boldsymbol{u}} \rrbracket \cdot\left\langle\boldsymbol{\sigma}_{\mathrm{b}}\right\rangle \cdot \boldsymbol{n}_{\mathrm{b}} d S+\int_{\Gamma_{\mathrm{UI}}} \llbracket \boldsymbol{w}_{\boldsymbol{u}} \rrbracket \cdot\langle\boldsymbol{\sigma}\rangle \cdot \boldsymbol{n}_{\mathrm{b}} d S \\
=\int_{B} \rho \boldsymbol{w}_{\boldsymbol{u}} \cdot \boldsymbol{b} d V+\int_{\Gamma_{\mathrm{N}}} \boldsymbol{w}_{\boldsymbol{u}} \cdot \overline{\boldsymbol{t}} d S \\
\int_{B}\left(w_{\tilde{e}} \tilde{e}+l_{\mathrm{c}}^{2} \nabla w_{\tilde{e}} \cdot \nabla \tilde{e}\right) d V+\int_{\Gamma_{\mathrm{UI}}} \llbracket w_{\tilde{e}} \rrbracket\langle c \boldsymbol{\nabla} \tilde{e}\rangle \cdot \boldsymbol{n}^{-} d S=\int_{B} w_{\tilde{e}} e d V .
\end{array}
$$

Compatibility equations $\boldsymbol{u}^{+}-\boldsymbol{u}^{-}=\mathbf{0}$ and $\tilde{e}^{+}-\tilde{e}^{-}=0$ are weakly added to the formulation (65) in order to enforce weakly the inter-element continuity on $\Gamma_{U I}$ and the stability of the system: the so-called symmetrisation and stability terms are added at the element interfaces. The first one ensures an optimal convergence rate with respect to the mesh size in the case of uncracked bodies while the second one ensures the stability throught a sufficiently high quadratic penalty term. Introducing $h_{\mathrm{s}}$ as the mesh characteristic size (this is not the mesh size $l_{\text {mesh }}$ since $h_{\mathrm{s}}$ depends on the polynomial approximation) and $\beta_{\mathrm{s}}$ the penalty parameter, the weak form (65) is reformulated as finding the field $(\boldsymbol{u}, \tilde{e})$ such that 


$$
\begin{array}{r}
\int_{B}\left(\rho \boldsymbol{w}_{\boldsymbol{u}} \cdot \ddot{\boldsymbol{u}}+\boldsymbol{\nabla} \boldsymbol{w}_{\boldsymbol{u}}: \boldsymbol{\sigma}\right) d V+\int_{\Gamma_{\mathrm{CI}}} \llbracket \boldsymbol{w}_{\boldsymbol{u}} \rrbracket \cdot\left\langle\boldsymbol{\sigma}_{\mathrm{b}}\right\rangle \cdot \boldsymbol{n}_{\mathrm{b}} d S+\int_{\Gamma_{\mathrm{U}}} \llbracket \boldsymbol{w}_{\boldsymbol{u}} \rrbracket \cdot\langle\boldsymbol{\sigma}\rangle \cdot \boldsymbol{n}_{\mathrm{b}} d S \\
+\int_{\Gamma_{\mathrm{UI}}} \llbracket \boldsymbol{u} \rrbracket \cdot\left\langle\mathcal{H}: \nabla \boldsymbol{w}_{\boldsymbol{u}}\right\rangle \cdot \boldsymbol{n}_{\mathrm{b}} d S+\int_{\Gamma_{\mathrm{UI}}} \llbracket \boldsymbol{w}_{\boldsymbol{u}} \rrbracket \otimes \boldsymbol{n}_{\mathrm{b}}:\left\langle\frac{\beta_{s}}{h_{s}} \mathcal{H}\right\rangle: \llbracket \boldsymbol{u} \rrbracket \otimes \boldsymbol{n}_{\mathrm{b}} d S \\
=\int_{B} \rho \boldsymbol{w}_{\boldsymbol{u}} \cdot \boldsymbol{b} d V+\int_{\Gamma_{\mathrm{N}}} \boldsymbol{w}_{\boldsymbol{u}} \cdot \overline{\boldsymbol{t}} d S, \\
\int_{B}\left(w_{\tilde{e}} \tilde{e}+l_{\mathrm{c}}^{2} \boldsymbol{\nabla} w_{\tilde{e}} \cdot \nabla \tilde{e}\right) d V+\int_{\Gamma_{\mathrm{UI}}} \llbracket w_{\tilde{e}} \rrbracket\left\langle l_{\mathrm{c}}^{2} \boldsymbol{\nabla} \tilde{e}\right\rangle \cdot \boldsymbol{n}^{-} d S+\int_{\Gamma_{\mathrm{UI}}} \llbracket \tilde{e} \rrbracket\left\langle l_{\mathrm{c}}^{2} \boldsymbol{\nabla} w_{\tilde{e}}\right\rangle \cdot \boldsymbol{n}_{\mathrm{b}} d S \\
+\int_{\Gamma_{\mathrm{UI}}} \llbracket w_{\tilde{e}} \rrbracket \boldsymbol{n}_{\mathrm{b}}\left\langle\frac{\beta_{s}}{h_{s}} l_{\mathrm{c}}^{2}\right\rangle \cdot \boldsymbol{n}_{\mathrm{b}} \llbracket \tilde{e} \rrbracket d S=\int_{B} w_{\tilde{e}} e d V,
\end{array}
$$

is satisfied for $\forall\left(\boldsymbol{w}_{\boldsymbol{u}}, w_{\tilde{e}}\right)$ kinematically admissible.

4.1.3. Finite element discretisation Now, the finite element discretisation is derived from the weak form (66). The displacement field $\boldsymbol{u}(\boldsymbol{X})$ and the non-local effective strain $\tilde{e}(\boldsymbol{X})$ are interpolated by the same nodal shape functions, as well as their respective trial functions in the volume element $B^{e}$ :

$$
\begin{array}{cc}
\boldsymbol{u}(\boldsymbol{X})=\sum_{a=1}^{N_{\mathrm{n}}} N^{a}(\boldsymbol{X}) \boldsymbol{u}^{a}, & \tilde{e}(\boldsymbol{X})=\sum_{a=1}^{N_{\mathrm{n}}} N^{a}(\boldsymbol{X}) \tilde{e}^{a}, \text { and } \\
\boldsymbol{w}_{\boldsymbol{u}}(\boldsymbol{X})=\sum_{a=1}^{N_{\mathrm{n}}} N^{a}(\boldsymbol{X}) \delta \boldsymbol{u}^{a}, & w_{\tilde{e}}(\boldsymbol{X})=\sum_{a=1}^{N_{\mathrm{n}}} N^{a}(\boldsymbol{X}) \delta \tilde{e}^{a},
\end{array}
$$

where $N^{a}(\boldsymbol{X})$ are the shape functions evaluated at node $a$, and $N_{\mathrm{n}}$ the number of nodes per volume elements. The unknowns are gathered inside nodal vectors $\boldsymbol{q}^{a}=\left[\left(\boldsymbol{u}^{a}\right)^{T} \tilde{e}^{a}\right]^{T}$ associated with each node $a$, and inside a global vector $\boldsymbol{q}=\left[\boldsymbol{u}^{T} \tilde{\boldsymbol{e}}^{T}\right]^{T}$ for all the unknowns of the mesh. Applying the discrete fields (67) to the weak form (66), and taking into account the inter-element discontinuities, we obtain this following set of differential equations

$$
\left\{\begin{array}{l}
\mathbf{M} \ddot{\boldsymbol{u}}+\boldsymbol{f}_{\boldsymbol{u} \text { int }}(\boldsymbol{q})+\boldsymbol{f}_{\boldsymbol{u} \mathrm{I}}(\boldsymbol{q})=\boldsymbol{f}_{\mathrm{ext}} \\
\boldsymbol{f}_{\tilde{e} \mathrm{int}}(\boldsymbol{q})-\boldsymbol{f}_{e \mathrm{int}}(\boldsymbol{q})+\boldsymbol{f}_{\tilde{e} \mathrm{I}}(\boldsymbol{q})=\mathbf{0}
\end{array} \quad \forall t>0\right.
$$

with the following initial conditions

$$
\boldsymbol{u}^{a}(t=0)=\mathbf{0}, \dot{\boldsymbol{u}}^{a}(t=0)=\dot{\boldsymbol{v}}_{0}^{a}
$$

where $\dot{\boldsymbol{v}}_{0}^{a}$ is the nodal vector of initial velocities. In the system (68), $\mathbf{M}$ corresponds to the discretised mass matrix; the vectors $\boldsymbol{f}_{\boldsymbol{u} \text { int }}, \boldsymbol{f}_{\boldsymbol{u}}$, and $\boldsymbol{f}_{\text {ext }}$ state respectively for the internal, interface, and external forces related to the displacement field, and the vectors $\boldsymbol{f}_{\tilde{e} \text { int }}, \boldsymbol{f}_{e \text { int }}$, and $\boldsymbol{f}_{\tilde{e} \text { I state }}$ respectively for the non-local internal, local internal, and interface forces related to the non-local field. The expressions, development, and computation of these vectors can be found in Appendix C.

The numerical properties connected to spatial discretisation of the presented framework inherit from those of the interior penalty formulation for elliptic problems [64], as discussed in [35]. During crack propagation, the dissipated energy and the crack path converge for unstructured meshes as the cohesive band model can be assimilated to an extrinsic cohesive zone, for which such a property 
has been shown [65]. As large systems need to be solved, the presented framework is implemented in parallel inside Gmsh [66] using the scalable face-based ghost element implementation, detailed in [30].

4.1.4. Damage to crack transition Inside this DG framework, both cracked and uncracked interface terms are integrated through the interface elements $\Gamma^{s}$, present from the beginning and inserted between each volume element. Once the criterion of crack initiation is reached at a Gauss point on $\Gamma_{\mathrm{UI}}$, this one is thereafter associated to $\Gamma_{\mathrm{CI}}$ and the DG terms are replaced by the cohesive one. Since only the term computation is modified at crack insertion, topology or mesh modification are avoided, which makes the implementation computationally efficient.

The damage is not directly used as a crack insertion criterion: it is based on the norm of the effective traction forces at the interface [30]. This criterion avoids simultaneous crack insertion around an element of quasi-constant damage value and adds a directional component. Practically, switch occurs if

$$
\frac{1}{1-D} \sqrt{\left\|\boldsymbol{t} \cdot \boldsymbol{n}_{\mathrm{b}}\right\|^{2}+\frac{1}{\beta_{\mathrm{c}}^{2}}\|\boldsymbol{t}-\| \boldsymbol{t} \cdot \boldsymbol{n}_{\mathrm{b}}\left\|\boldsymbol{n}_{\mathrm{b}}\right\|^{2}}>\hat{\sigma}_{\mathrm{c}} \text { and if } \boldsymbol{t} \cdot \boldsymbol{n}_{\mathrm{b}}>0
$$

where $\|\bullet\|$ states for the norm, $\boldsymbol{t}=\boldsymbol{\sigma} \cdot \boldsymbol{n}_{\mathrm{b}}$ for the surface traction, $\hat{\sigma}_{\mathrm{c}}$ for the critical effective stress value, and $\beta_{\mathrm{c}}$ for the ratio between opening mode I and mode II. Practically, this parameter is set as the ratio between fracture toughness of mode I and mode II. A scattering is applied on $\hat{\sigma}_{\mathrm{c}}$ for explicit simulations in order to capture crack propagation as proposed by [67].

During crack propagation, once the majority of the Gauss points of an interface element have switched to the cohesive band model, the CBM is also introduced on the remaing Gauss points of that interface element although Eq. (70) is not satisfied yet. By this way, spurious rotation of elements around one point/line is avoided. Once an interface element is completely opened, damage evolution is blocked inside the neighbouring bulk elements as they should be elastically unloaded. Moreover, the bulk local equivalent strain evolution, $e$ of Eq. (38), is limited to the maximal value it ever reached in order to avoid spurious damage spread and crack insertion around the element, but also to force elastic unloading. In case of crack closing and penetration, a normal quadratic penalty force is added. This form is preferred to a linear one in order to avoid tangent discontinuities and improve convergence.

\subsection{Numerical time integration}

The set of differential equations (68) has to be integrated. On the one hand, under quasi-static loading assumption, it can be integrated in a fully coupled iterative procedure as detailed in Section 4.2.1. On the other hand, under dynamic loading a coupled explicit-implicit scheme, as explained in Section 4.2.2, can be used.

4.2.1. Implicit integration scheme Under the assumption of quasi-static loading (i.e. if inertia effects are negligible), the inertia or acceleration force term $\mathbf{M} \ddot{u}$ in the set of differential equations (68) can be neglected. The time has therefore only a role of chronological ordering and the system is integrated in an incremental way. An iterative Newton-Raphson procedure is used to compute the 
equilibrium state at each discrete time step $t^{n}$. The system (68) is rewritten in the residual form

$$
\boldsymbol{r}\left(\boldsymbol{q}^{k}\left(t^{n}\right), t^{n}\right)=\left\{\begin{array}{l}
\boldsymbol{f}_{\boldsymbol{u} \text { int }}\left(\boldsymbol{q}^{k}\right)+\boldsymbol{f}_{\boldsymbol{u} \mathrm{I}}\left(\boldsymbol{q}^{k}\right)-\boldsymbol{f}_{\mathrm{ext}}\left(t^{n}\right) \\
\boldsymbol{f}_{\tilde{e} \text { int }}\left(\boldsymbol{q}^{k}\right)-\boldsymbol{f}_{e \text { int }}\left(\boldsymbol{q}^{k}\right)+\boldsymbol{f}_{\tilde{e} \mathrm{I}}\left(\boldsymbol{q}^{k}\right)
\end{array}\right.
$$

where $\boldsymbol{r}\left(\boldsymbol{q}^{k}\left(t^{n}\right), t^{n}\right)$ corresponds to the force unbalance at time step $t^{n}$ and $\boldsymbol{q}^{k}\left(t^{n}\right)$ correponds to the field value at iteration $k$. The Newton-Raphson linearisation reads

$$
\boldsymbol{r}\left(\boldsymbol{q}^{k+1}, t^{n}\right)=\boldsymbol{r}\left(\boldsymbol{q}^{k}, t^{n}\right)+\left.\frac{\partial r\left(\boldsymbol{q}^{k}, t^{n}\right)}{\partial \boldsymbol{q}}\right|_{\boldsymbol{q}^{k}} \delta \boldsymbol{q}=\mathbf{0}
$$

Or, again using the residual form (71),

$$
\underbrace{\frac{\partial \boldsymbol{f}_{\boldsymbol{u} \text { int }}}{\partial \boldsymbol{u}}}_{\mathbf{K}_{u \boldsymbol{u} \text { int }}} \delta \boldsymbol{u}+\underbrace{\frac{\partial \boldsymbol{f}_{\boldsymbol{u} \text { int }}}{\partial \tilde{\boldsymbol{e}}}}_{\mathbf{K}_{\boldsymbol{u} \tilde{e} \text { int }}} \delta \tilde{\boldsymbol{e}}+\underbrace{\frac{\partial \boldsymbol{f}_{\boldsymbol{u} \mathrm{I}}}{\partial \boldsymbol{u}}}_{\mathbf{K}_{u \boldsymbol{u} \mathrm{I}}} \delta \boldsymbol{u}+\underbrace{\frac{\partial \boldsymbol{f}_{\boldsymbol{u}} \mathrm{I}}{\partial \tilde{\boldsymbol{e}}}}_{\mathbf{K}_{\boldsymbol{u} \tilde{e} \mathrm{I}}} \delta \tilde{\boldsymbol{e}}=\boldsymbol{f}_{\mathrm{ext}}\left(t^{n}\right)-\boldsymbol{f}_{\boldsymbol{u} \text { int }}\left(\boldsymbol{q}^{k}\right)-\boldsymbol{f}_{\boldsymbol{u} \mathrm{I}}\left(\boldsymbol{q}^{k}\right),
$$

and,

$$
\begin{aligned}
& \underbrace{\frac{\partial \boldsymbol{f}_{\tilde{e} \text { int }} \partial \boldsymbol{u}}{\boldsymbol{u}}}_{\mathbf{K}_{\tilde{e} \boldsymbol{u} \text { int }}} \delta \boldsymbol{u}+\underbrace{\frac{\partial \boldsymbol{f}_{\tilde{e}} \text { int }}{\partial \tilde{\boldsymbol{e}}}}_{\mathbf{K}_{\tilde{e} \tilde{e} \text { int }}} \delta \tilde{\boldsymbol{e}}-\underbrace{\frac{\partial \boldsymbol{f}_{e} \text { int }}{\partial \boldsymbol{u}}}_{\mathbf{K}_{e \boldsymbol{u} \text { int }}} \delta \boldsymbol{u}-\underbrace{\frac{\partial \boldsymbol{f}_{e} \text { int }}{\partial \tilde{\boldsymbol{e}}}}_{\mathbf{K}_{e \tilde{e} \text { int }}} \delta \tilde{\boldsymbol{e}}+\underbrace{\frac{\partial \boldsymbol{f}_{\tilde{e} \mathrm{I}}}{\partial \boldsymbol{u}}}_{\mathbf{K}_{\tilde{e} \boldsymbol{u} \mathrm{I}}} \delta \boldsymbol{u}+\underbrace{\frac{\partial \boldsymbol{f}_{\tilde{e} \mathrm{I}}}{\partial \tilde{\boldsymbol{e}}}}_{\mathbf{K}_{\tilde{e} \tilde{e} \mathrm{I}}} \delta \tilde{\boldsymbol{e}} \\
& =\boldsymbol{f}_{e \mathrm{int}}\left(\boldsymbol{q}^{k}\right)-\boldsymbol{f}_{\tilde{e} \mathrm{int}}\left(\boldsymbol{q}^{k}\right)-\boldsymbol{f}_{\tilde{e} \mathrm{I}}\left(\boldsymbol{q}^{k}\right),
\end{aligned}
$$

in which the matrices $\mathbf{K} \ldots$ are the contributions to the stiffness matrix. Their expressions are reported in Appendix D. The system (73)-(74) can then be rewritten as

$$
\begin{aligned}
& {\left[\begin{array}{cc}
\mathbf{K}_{\boldsymbol{u} \boldsymbol{u} \text { int }}+\mathbf{K}_{\boldsymbol{u} \boldsymbol{u} \mathrm{I}} & \mathbf{K}_{\boldsymbol{u} \tilde{e} \mathrm{int}}+\mathbf{K}_{\boldsymbol{u} \tilde{e} \mathrm{I}} \\
\mathbf{K}_{\tilde{e} \boldsymbol{u} \boldsymbol{i n t}}-\mathbf{K}_{e \boldsymbol{u} \mathrm{int}}+\mathbf{K}_{\tilde{e} \boldsymbol{u} \mathrm{I}} & \mathbf{K}_{\tilde{e} \tilde{e} \tilde{\mathrm{int}}}-\mathbf{K}_{e \tilde{e} \mathrm{int}}+\mathbf{K}_{\tilde{e} \tilde{e} \mathrm{I}}
\end{array}\right] \underbrace{\left[\begin{array}{c}
\delta \boldsymbol{u} \\
\delta \tilde{\boldsymbol{e}}
\end{array}\right]}_{\delta \boldsymbol{q}}} \\
& =\left[\begin{array}{c}
\boldsymbol{f}_{\text {ext }}\left(t^{n}\right)-\boldsymbol{f}_{\boldsymbol{u} \text { int }}\left(\boldsymbol{q}^{i}\right)-\boldsymbol{f}_{\boldsymbol{u} \mathrm{I}}\left(\boldsymbol{q}^{i}\right) \\
\boldsymbol{f}_{e \text { int }}\left(\boldsymbol{q}^{i}\right)-\boldsymbol{f}_{\tilde{e} \text { int }}\left(\boldsymbol{q}^{i}\right)-\boldsymbol{f}_{\tilde{e} \mathrm{I}}\left(\boldsymbol{q}^{i}\right)
\end{array}\right],
\end{aligned}
$$

and its resolution for $\delta \boldsymbol{q}$ gives the new guess $\boldsymbol{q}^{k+1}=\boldsymbol{q}^{k}+\delta \boldsymbol{q}$ at the end of the iteration $k$. This Newton-Raphson procedure has to be repeated until the convergence is reached. To ensure a wellconditioned system, a ratio is applied between the force equilibrium and the non-local system.

The main advantage of the fully coupled implicit solving is that it is unconditionally stable. However, implicit iterations are time-consuming. Convergence needs small time steps and cannot always be reached, especially in case of local snap-backs, unstable crack propagations, or in the case of the arising of new rigid body modes.

4.2.2. Coupled explicit-implicit integration scheme The first set of equations in the system (68) can be integrated with an explicit integration scheme. The Hulbert-Chung [68] time integration scheme 
is considered here because of its second-order accuracy and its controlled numerical damping. From the solution at time $t^{n}$, the solution at the next time step $t^{n+1}=t^{n}+\Delta t$ is obtained by

$$
\begin{aligned}
& \ddot{\boldsymbol{u}}^{a, n+1}=\frac{1}{1-\alpha_{\mathrm{M}}} \sum_{b=1}^{N_{\mathrm{n}}} \mathbf{M}^{b a-1}\left(\boldsymbol{f}_{\mathrm{ext}}^{b, n}-\boldsymbol{f}_{\boldsymbol{u} \text { int }}^{b, n}-\boldsymbol{f}_{\boldsymbol{u} \mathrm{I}}^{b, n}\right)-\frac{\alpha_{\mathrm{M}}}{1-\alpha_{\mathrm{M}}} \ddot{\boldsymbol{u}}^{a, n}, \\
& \dot{\boldsymbol{u}}^{a, n+1}=\dot{\boldsymbol{u}}^{a, n}+\left(1-\gamma_{\mathrm{M}}\right) \Delta t \ddot{\boldsymbol{u}}^{a, n}+\gamma_{\mathrm{M}} \Delta t \ddot{\boldsymbol{u}}^{a, n+1}, \text { and } \\
& \boldsymbol{u}^{a, n+1}=\boldsymbol{u}^{a, n}+\Delta t \dot{\boldsymbol{u}}^{a, n}+\left(\frac{1}{2}-\beta_{\mathrm{M}}\right) \Delta t^{2} \ddot{\boldsymbol{u}}^{a, n}+\beta_{\mathrm{M}} \Delta t^{2} \ddot{\boldsymbol{u}}^{a, n+1},
\end{aligned}
$$

with the parameters $\alpha_{\mathrm{M}}, \beta_{\mathrm{M}}$ and $\gamma_{\mathrm{M}}$ depending on the sought numerical dissipation [68].

As there is no mass matrix associated to the non-local equation, the remaining system has to be solved implicitly. By linearising the non-local equation (68) around the state $\boldsymbol{q}^{i}$ obtained at a given iteration $i$, one has in terms of the non-local increment $\delta \tilde{e}$ :

$$
\mathbf{K}_{\tilde{e} \tilde{e}} \delta \tilde{\boldsymbol{e}}=\boldsymbol{f}_{\text {e int }}\left(\boldsymbol{q}^{i}\right)-\boldsymbol{f}_{\tilde{e} \text { int }}\left(\boldsymbol{q}^{i}\right)-\boldsymbol{f}_{\tilde{e} \mathrm{I}}\left(\boldsymbol{q}^{i}\right)
$$

where

$$
\mathbf{K}_{\tilde{e} \tilde{e}}=\underbrace{\frac{\partial \boldsymbol{f}_{\tilde{e} \mathrm{int}}}{\partial \tilde{\boldsymbol{e}}}}_{\mathbf{K}_{\tilde{e} \tilde{e} \mathrm{int}}}-\underbrace{\frac{\partial \boldsymbol{f}_{e \mathrm{int}}}{\partial \tilde{\boldsymbol{e}}}}_{\mathbf{K}_{e \tilde{e} \mathrm{int}}}+\underbrace{\frac{\partial \boldsymbol{f}_{\tilde{e} \mathrm{I}}}{\partial \tilde{\boldsymbol{e}}}}_{\mathbf{K}_{\tilde{e} \tilde{e} \mathrm{I}}} .
$$

The expressions for these stiffness matrices can be found in Appendix D. Taking advantage of the unconditional stability of the implicit resolution of the non-local equations, these equations are solved every 100 to 1000 time steps to avoid computational over-costs.

The main advantages of this weakly coupled scheme are its low-cost of step computation and the lower restrictions due to convergence problem. However, a stability criterion on the time step has to be respected to ensure method stability. Indeed, the explicit time integration of the first set of equations in Eq. (68) using the Hulbert-Chung algorithm is conditionally stable. The time step $\Delta t$ is bounded to satisfy a Courant-Friedrichs-Lewy (CFL) condition. The stability criterion depends on the spatial DG-discretisation mesh size $h_{\mathrm{s}}$, the sound speed $c_{\mathrm{s}}$, the stability parameter $\beta_{\mathrm{s}}$, and the stability non-dimensional frequency of the time integration method $\Omega_{\mathrm{s}} \leq 2$ [68]. The CFL-condition reads $\Delta t<\frac{h_{\mathrm{s}} \Omega_{\mathrm{s}}}{2 c_{\mathrm{s}} \sqrt{\beta_{\mathrm{s}}}}[26]$.

\section{NUMERICAL APPLICATIONS AND TRIAXIALITY EFFECTS}

In this section, the numerical properties of the developed non-local damage/cohesive band model $(\mathrm{CDM} / \mathrm{CBM})$ transition are investigated through numerical examples. In particular, the impact of the triaxiality effect is studied. During this section, the numerical prediction of the hybrid CDM/CBM are compared with the non-local scheme without crack insertion (N-L), and with an hybrid scheme involving a (classical) cohesive zone model (CDM/CZM) as developed in [35]. When available, the numerical predictions are compared with phase field results or validated with experimental results from the literature. 


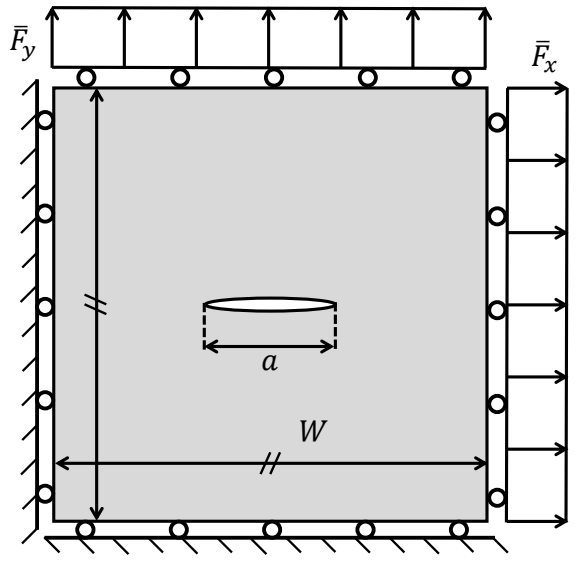

(a)

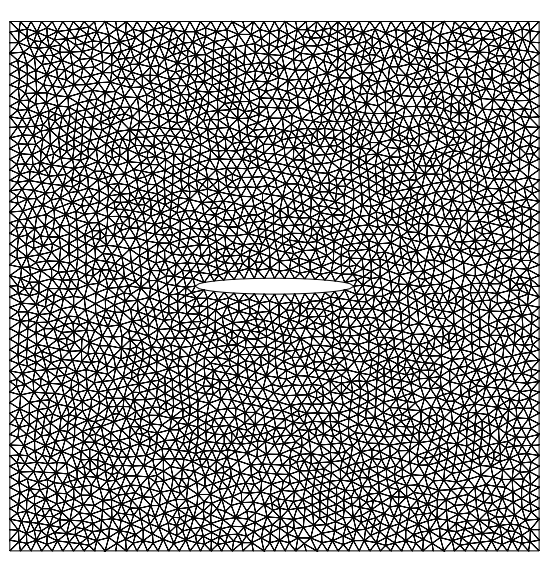

(b)

Figure 8. (a) The flat holed plate geometry with $W=40 \mathrm{~mm}$ and $a=12 \mathrm{~mm}$; and (b) its mesh.

\subsection{Slit plate}

At first, the CDM/CBM is applied on a simple 2D slit specimen. After having verified the mesh insensitivity, the specimen is submitted to various loading ratio coefficients in order to show the sensibility of the method to triaxiality effects contrarily to the CDM/CZM model.

The 2D specimen consists in a square plate of length $W=40 \mathrm{~mm}$. A thin elliptical hole of major radius $a / 2=6 \mathrm{~mm}$ and minor one $a / 20$ is introduced at its center. The plate is under plane strain condition along its thickness $t$. The geometry is illustrated in Fig. 8(a). The plate is loaded by applying a force $\bar{F}_{\mathrm{y}}$ on the top boundary and a force $\bar{F}_{\mathrm{x}}=k \bar{F}_{\mathrm{y}}$ on the right boundary. The plate edges are constrained to stay straight while the ratio $k$ between the vertical and horizontal force is kept constant during each test. By varying the proportion between the vertical and horizontal loading conditions, the stress triaxiality $T$ (ratio $\frac{\operatorname{tr} \sigma}{3 \sigma^{\mathrm{vm}}}$ between the pressure and equivalent von Mises stress $\sigma^{\mathrm{vm}}$ ) inside the system evolves. The plate is uniformly meshed with 70586 -node triangles of $0.8 \mathrm{~mm}$ mean size (unless otherwise mentioned) as shown in Fig. 8(b). These elements involve quadratic shape functions for the displacement and the non-local field discretisations. The system is solved using the implicit scheme detailed in Section 4.2.1 completed by a path following method in order to capture a possible snap-back.

The material behaviour is assimilated to an isotropic non-local damage linear elastic model, combined with a cohesive band model as described in Section 2.3 or with a cohesive zone as in [35] for the sake of comparison. The material parameters are reported in Table I [60, 35]. The damage to crack transition parameters for the hybrid CDM/CBM and CDM/CZM are listed in Table II. The damage to crack transition is triggered by an effective critical stress $\hat{\sigma}_{\mathrm{c}}$, which is identical for both hybrid frameworks. An approximate corresponding value ${ }^{\dagger}$ of damage $D_{\mathrm{c}}$ at which transition occurs can be obtained by comparison with the one-dimensional case. The effective critical stress $\hat{\sigma}_{\mathrm{c}}$ is here chosen to insert crack at a damage values around 0.75 for a uniaxial loading, in order

\footnotetext{
${ }^{\dagger}$ This is an approximate value since it is obtained for the stress triaxiality which corresponds to a uniaxial loading
} 
Table I. Material properties for short glass-fiber-reinforced polypropylene.

\begin{tabular}{lcl}
\hline Elastic model properties, Eq. (34) & & \\
$\quad$ Young's Modulus & $E$ & $3.2[\mathrm{GPa}]$ \\
$\quad$ Poisson ratio & $\nu$ & 0.28 \\
\hline Damage model properties, Eq.(40): & & \\
$\quad$ Initial damage threshold & $\kappa_{\mathrm{i}}$ & 0.011 \\
$\quad$ Failure equivalent strain & $\kappa_{\mathrm{c}}$ & 0.50 \\
Damage exponents & $\alpha$ & 5.0 \\
& $\beta$ & 0.75 \\
\hline Non-local model properties, Eq.(38): & & \\
$\quad$ Non-local length & $l_{\mathrm{c}}^{2}$ & $2.0\left[\mathrm{~mm}^{2}\right]$ \\
\hline
\end{tabular}

Table II. Damage to crack transition parameters for the flat holed plate.

\begin{tabular}{lll}
\hline Crack insertion criterion, Eq. (70): & & \\
$\quad$ Effective critical stress & $\hat{\sigma}_{\mathrm{c}}$ & $280[\mathrm{MPa}]$ \\
Critical damage & $D_{\mathrm{c}}$ & 0.85 \\
Mix-mode ratio & $\beta_{\mathrm{c}}$ & 0.87 \\
\hline Cohesive band model: & & \\
$\quad$ Band thickness & $h_{\mathrm{b}}$ & $7.6[\mathrm{~mm}]$ \\
\hline Cohesive zone model [35]: & & \\
$\quad$ Remaining energy & $G_{\mathrm{c}}$ & $21.4\left[\mathrm{~kJ} / \mathrm{m}^{2}\right]$ \\
\hline
\end{tabular}

to highlight the differences between the three models. The fracture energy $G_{\mathrm{c}}$ of the cohesive zone model, corresponding to the remaining energy needed to be dissipated after crack transition, is extracted from the one-dimensional simulation as shown in Fig. 6(a). The values used here are slightly higher than the corresponding ones in [35] because the non-local length is constant whistle it was variable in [35]. For the cohesive band model, only the band thickness is required and has been computed in Section 3.

5.1.1. Mesh insensitivity The mesh insensitivity of the CDM/CBM framework is here investigated. The force-displacement curves and the total dissipated energy are compared for different mesh sizes. The involved simulations have constrained crack paths along the symmetry planes in order to focus on the cohesive band effect only since other framework features are mesh-independent. Indeed, because a non-local model is used, no mesh-dependency is expected during the softening before crack insertion [10], providing that the mesh size is sufficiently small in regards to the characteristic length. Moreover, the crack path should converge for extrinsic cohesive zone [65] during the crack propagation.

The problem is simulated with the 3 different meshes shown in Fig. 9. The vertical loading force evolutions in terms of the vertical displacement $\bar{u}_{\mathrm{y}}$ and for a loading ratio $k=0$ are shown in Fig. 10(a) for the different mesh sizes. The three curves are similar, although unloading-loading cycles are observed at crack insertion due to the combination of damage freezing around cracked interfaces and the use of the path-following method. The depth of those cycles is more pronounced with increasing mesh size. Moreover, a larger mesh size delays the crack insertion since the stress at the crack tip is lower at the first Gauss point. The total dissipated energy in terms of the mesh size 


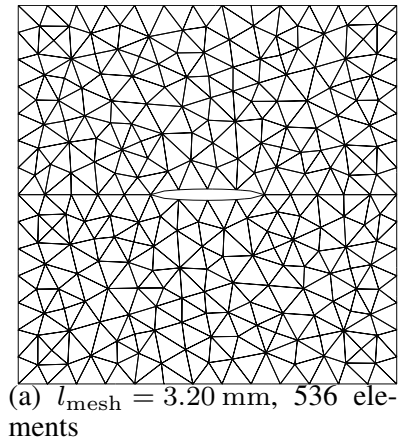

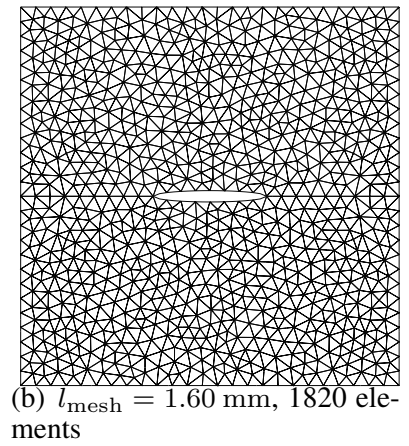

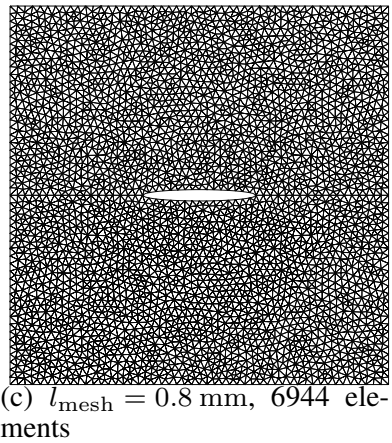

ments

Figure 9. The mesh of the holed plate with different uniform mesh sizes $l_{\text {mesh }}$.

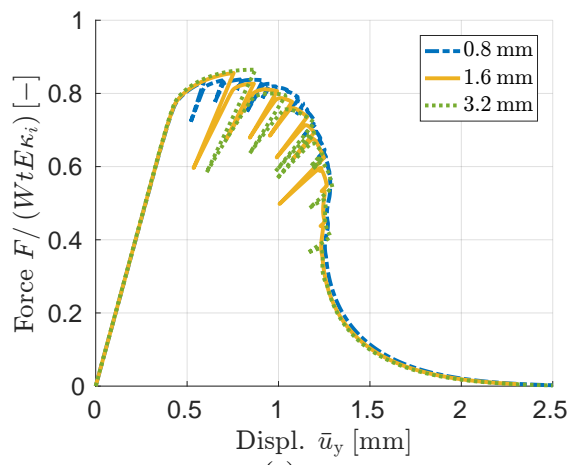

(a)

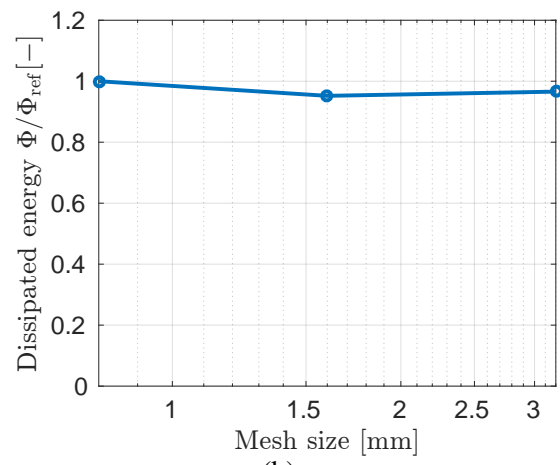

(b)

Figure 10. (a) Vertical loading force in terms of the vertical displacement $\bar{u}_{\mathrm{y}}$ for the different mesh sizes and (b) the corresponding total dissipated energy. The reference dissipated energy $\Phi_{\text {ref }}$ is here the value associated with the finest mesh.

is represented in Fig. 10(b) and no signifiant variation is observed with respect with the mesh size. The difference results from the numerical approximations (loading increments).

5.1.2. Study of the triaxiality effects The plate is now loaded with different ratio coefficients $k=[-1 / 2 ; 0 ; 1 / 2]$ and the simulation results obtained by using the non-local model (N-L), the hybrid CDM/CBM model, and the hybrid CDM/CZM model are compared. The three models are identical before crack insertion. The damage fields are represented just before first crack insertion in Fig. 11(a) to 11(c) for the different values of $k$. The three distributions have a similar pattern but are more developed for the lower value of $k$ since the transition occurs later in those less constrained states. The corresponding triaxiality distributions are shown in Fig. 11(d) to 11(f) and, as expected, are different for each case and increases with $k$.

After initiation, the cracks propagate near the median line of the plate for both hybrid frameworks while strains and damage continue to grow for simulations involving the non-local model only. The final damage distributions at material failure (when the vertical force drops to 0 ) are presented in the deformed configurations in Fig. 12(a) to 12(i). Globally, for a given numerical model, failure 


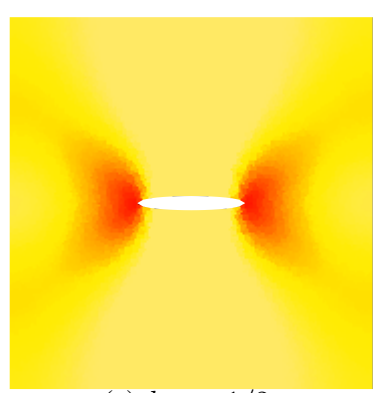

(a) $k=-1 / 2$

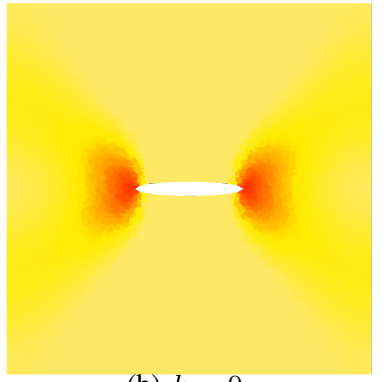

(b) $k=0$ 0.5 $D^{0}$

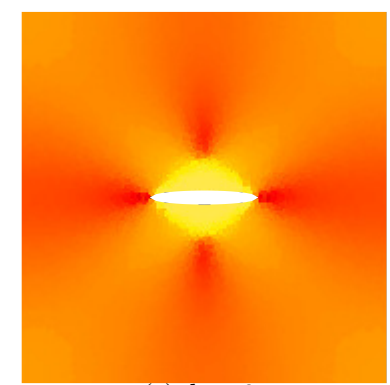

(e) $k=0$

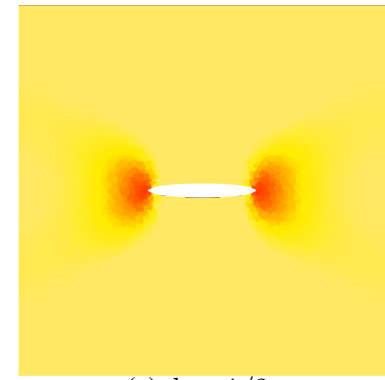

(c) $k=1 / 2$

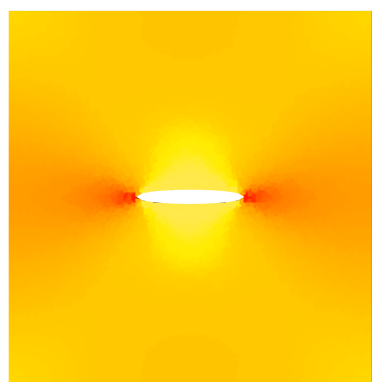

(d) $k=-1 / 2$ $T^{-0.75} \quad 0.6 \quad 2$

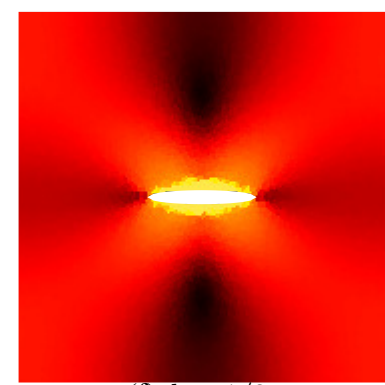

(f) $k=1 / 2$

Figure 11. (a-c) Damage $D$ field and (d-f) triaxiality $T$ state at first crack insertion for different values of $k$.

damage fields tend to be more spread for smaller values of $k$. For the non-local model, high damage values spread around the fictitious crack surface due to the highly stretched elements while damage evolution is stopped behind the crack tip with the hybrid frameworks. For a given value of $k$, the final crack opening is higher with the cohesive band model than with the cohesive zone, which expresses a less brittle behaviour for the band model.

The evolutions of the vertical loading forces $F_{\mathrm{y}}$ in terms of the vertical displacements $\bar{u}_{\mathrm{y}}$ are reported in Fig. 13(a), 13(c), and 13(e) for the three frameworks. Increasing $k$ tends globally to rise the peak stress value but to decrease the displacement value at which this peak is reached. When comparing the models, no difference is observable before crack insertion as expected. During crack propagation, the CDM/CZM exhibits a more brittle behaviour than the hybrid CDM/CBM and than the non-local model. This is explained by the fact that CDM/CZM does not account for the triaxiality induces by the out-of-plane plane strain state and by the loading ratio $k$. The maximal stress value reached is higher for the non-local model than for the CDM/CBM but this is balanced by a longer response tail with the CBM. This longer tail results in a larger crack opening at failure than with the pure non-local model. This could be avoided by considering a different damage evolution law in the CBM after shift has occured, but this is out of the scope of this paper.

The corresponding dissipated energy evolutions are shown in Fig. 13(b), 13(d), and 13(f) where $\Phi_{\text {ref }}$ is defined as being the energy dissipated by the pure non-local model with $k=0$. During the crack propagation, the results of the hybrid $\mathrm{CDM} / \mathrm{CBM}$ predictions remain closer from those of the 


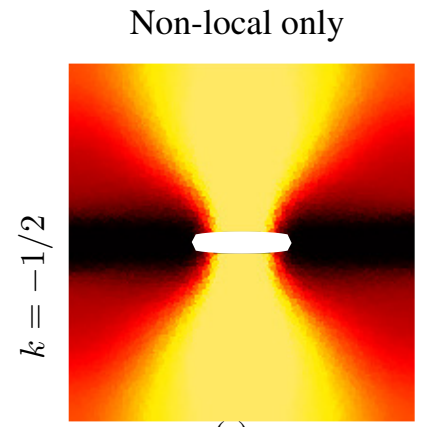

(a)

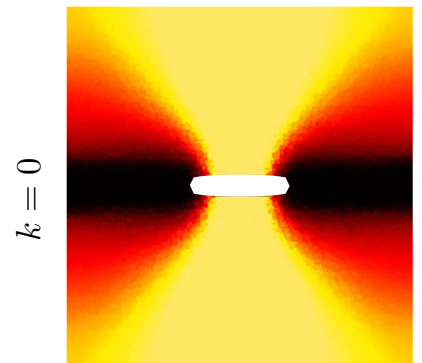

(d)

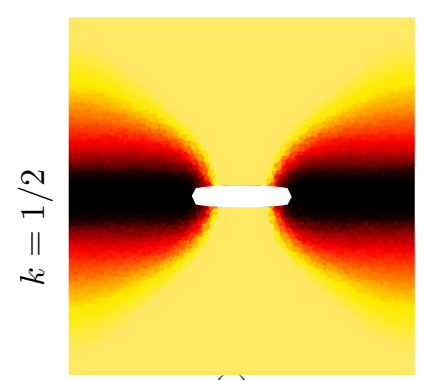

(g)
$\mathrm{CDM} / \mathrm{CBM}$

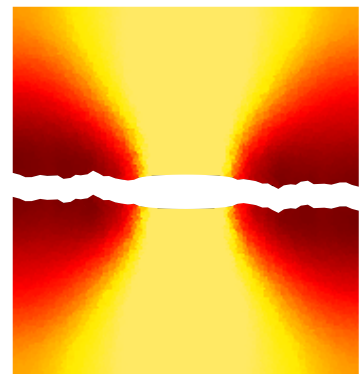

(b)

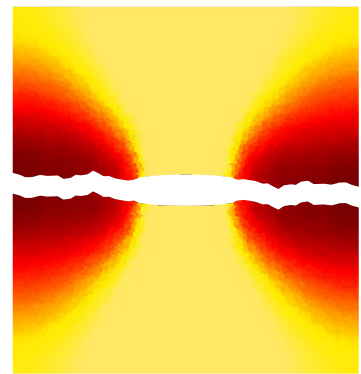

(e)

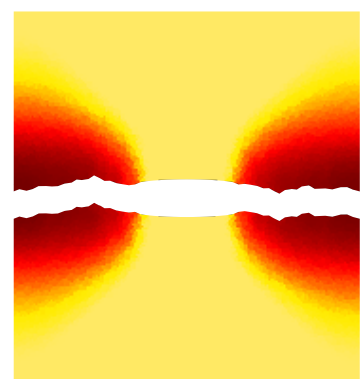

(h)
$\mathrm{CDM} / \mathrm{CZM}$

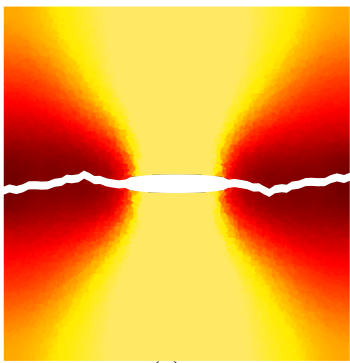

(c)

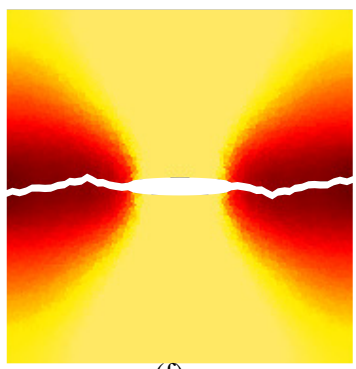

(f)

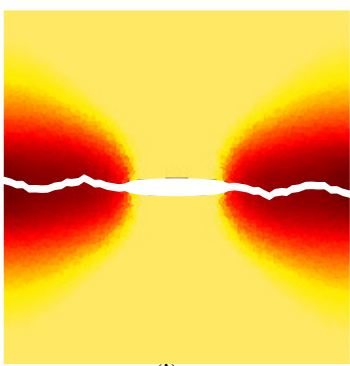

(i)

$D^{0}$

0.5

Figure 12. Damage field at material failure for the pure non-local, CDM/CBM, and CDM/CZM frameworks, and for different values of the loading ratio $k$.

non-local model than the prediction of the CDM/CZM until the sample is totally cracked. Despite the two dissipation rates differ, the final total amount of dissipated energy are coherent between the non-local model and the CDM/CBM for the different triaxiality states. The CDM/CBM differ from the non-local model by maximum 5\%; this difference can be partially explained by the non-straight crack pattern which increases the crack surface and thus the dissipation.

Besides, the more brittle behaviour of CDM/CZM previously mentioned reflects that the $\mathrm{CDM} / \mathrm{CZM}$ is unable to dissipate the correct amount of energy, resulting in one order of magnitude higher in the error for all tests (i.e. around 30\%). This is explained by the inability of the CZM to incorporate stress triaxiality effects since the fracture energy of the cohesive zone is fixed in advance (here, calibrated on a one-dimensional uniaxial tension). Moreover, the crack criterion is based on an effective stress. Therefore, the damage value at crack insertion is not guaranteed. To 


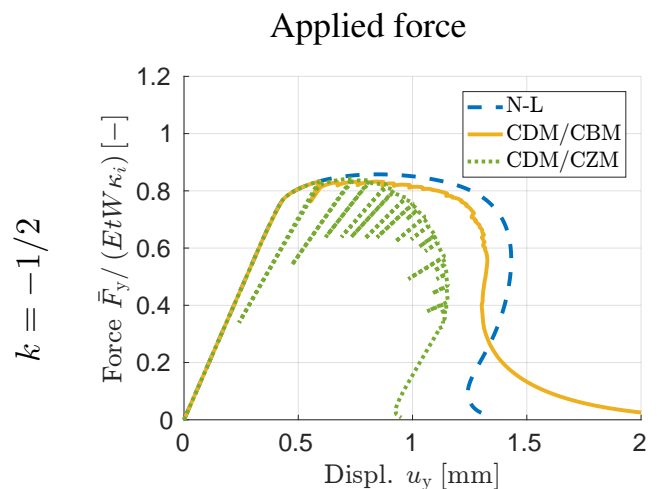

(a)

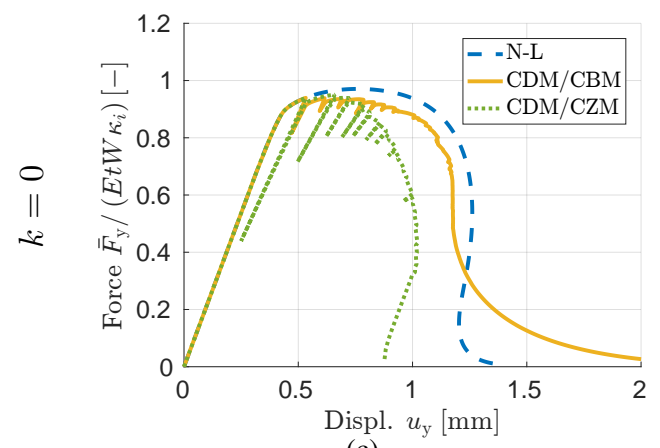

(c)

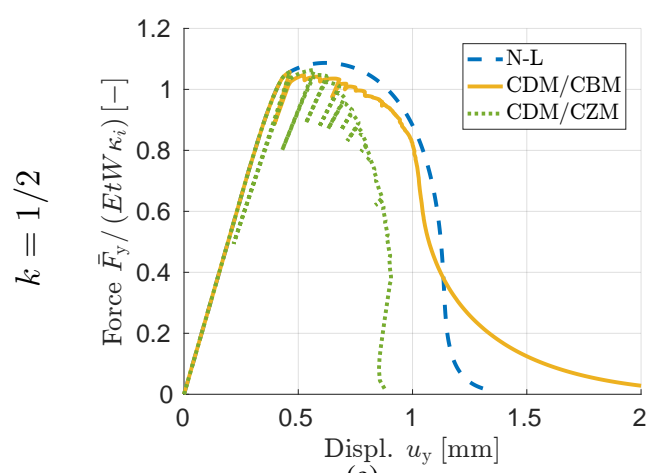

(e)

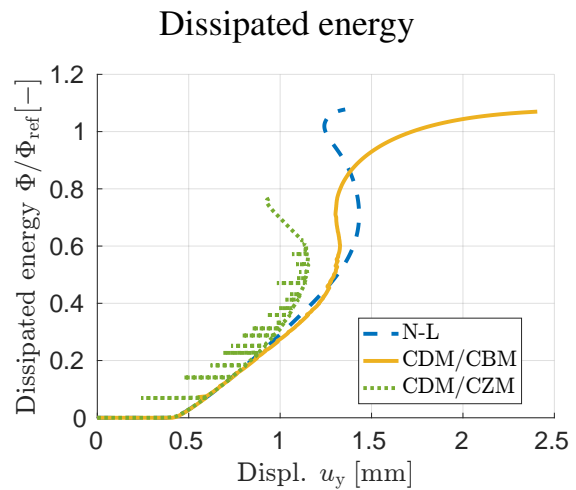

(b)

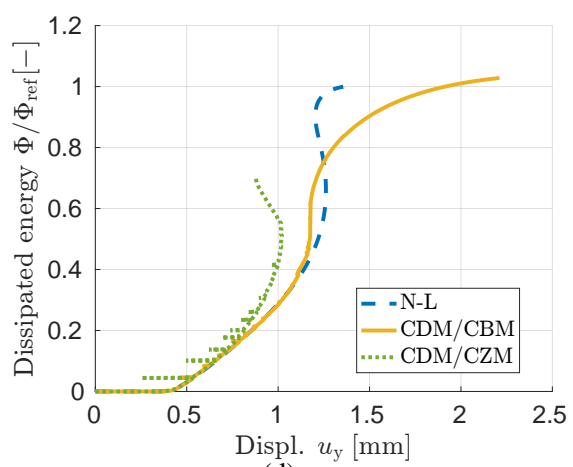

(d)

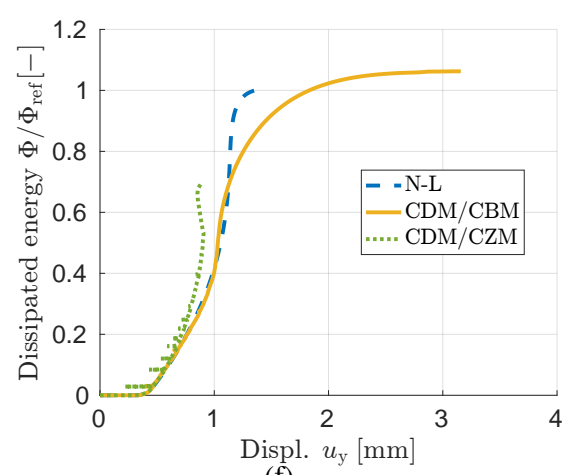

(f)

Figure 13. Vertical loading force $F_{\mathrm{y}}$ (left) and dissipated energy $\Phi$ (right) $v$ s. vertical displacement $\bar{u}_{\mathrm{y}}$ evolutions for the pure non-local, CDM/CBM, and CDM/CZM frameworks, and for different values of the loading ratio $k$. The reference dissipated energy $\Phi_{\text {ref }}$ is here the value associated with the pure non-local model and $k=0$.

overcome these problems, the CZM could be calibrated for each specific case, which is avoided by the CDM/CBM. Indeed, the cohesive band thickness does not depend on damage insertion value. Nevertheless, this approach would not account for the variation of stress triaxiality state during the sample loading, whistle the CDM/CBM framework includes those triaxiality effects and therefore is able to follow the variation of dissipated energy. 


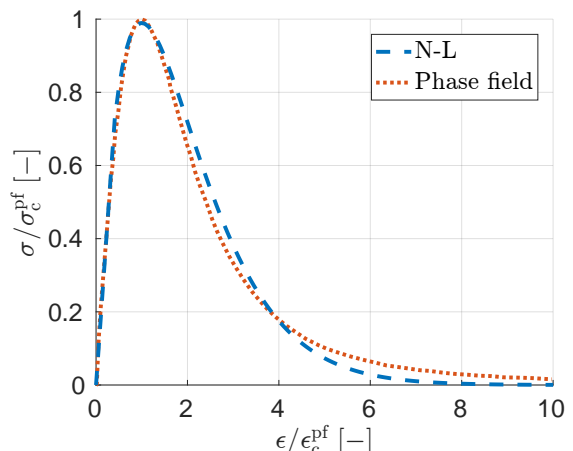

(a)

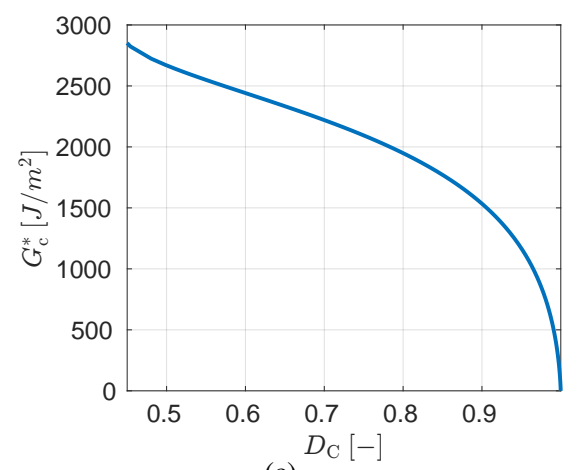

(c)

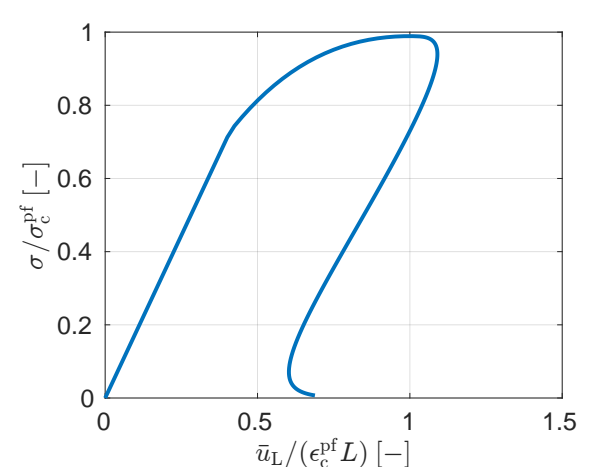

(b)

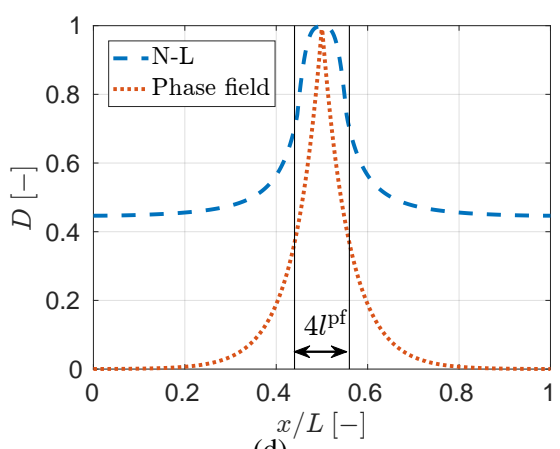

(d)

Figure 14. Stress response (a) for a one-dimensional uniform strain state, and (b) with localisation in a bar of length $L=0.5 \mathrm{~mm}$. (c) Corresponding fracture energy which remains to be dissipated in terms of crack damage insertion. (d) Comparison between non-local damage profile and the idealised phase field one; the vertical dashed lines represent the phase field smeared crack of width $4 l^{\mathrm{pf}}$.

\subsection{Single edge notched specimens}

The hybrid CDM/CBM framework is now compared to the phase field framework applied to brittle fracture $[11$, e.g.]. To this end, the single edge notched specimens studied in $[59,12]$ are considered under tensile and shear loading conditions.

5.2.1. Material parameters The elastic material properties reported in [59, 12] are a Young's modulus $E=210.0 \mathrm{GPa}$ and a Poisson ratio $\nu=0.3$. The critical energy release rate is $G_{\mathrm{c}}=$ $2700 \mathrm{~J} / \mathrm{m}^{2}$ and the length scale associated to the phase field results is $l^{\mathrm{pf}}=15 \times 10^{-3} \mathrm{~mm}$. The plane strain state is assumed.

First, the material parameters of the underlying non-local damage model of the hybrid $\mathrm{CDM} / \mathrm{CBM}$ framework have to be calibrated with respect to the brittle behaviour studied in $[59,12]$. In particular, the non-local length, the damage model, and the crack insertion properties have to be determined. The elastic properties are taken as such, see Table III. The damage model is parametrised in order to fit the one-dimensional homogeneous response related to the phase field 
Table III. Material properties for the single edge notched specimen tests.

\begin{tabular}{lll}
\hline Elastic model properties, Eq. (34): & & \\
$\quad$ Young's Modulus & $E$ & $210.0[\mathrm{GPa}]$ \\
$\quad$ Poisson ratio & $\nu$ & 0.3 \\
\hline Damage model properties, Eq.(40): & & \\
$\quad$ Initial damage threshold & $\kappa_{\mathrm{i}}$ & 0.0049 \\
$\quad$ Failure equivalent strain & $\kappa_{\mathrm{c}}$ & 0.49 \\
Damage exponents & $\alpha$ & 40.0 \\
& $\beta$ & 0.0 \\
\hline Non-local model properties, Eq.(38): & & \\
$\quad$ Non-local length & $l_{\mathrm{c}}$ & $16.5 \times 10^{-3}[\mathrm{~mm}]$ \\
\hline
\end{tabular}

Table IV. Damage to crack transition parameters for the single edge notched specimen tests.

\begin{tabular}{lll}
\hline Crack insertion criterion, Eq. (70): & & \\
Effective critical stress & $\hat{\sigma}_{\mathrm{c}}$ & $5.9[\mathrm{GPa}]$ \\
Critical damage & $D_{\mathrm{c}}$ & 0.85 \\
Mix-mode ratio & $\beta_{\mathrm{c}}$ & 0.87 \\
\hline Cohesive band model: & & \\
$\quad$ Band thickness & $h_{\mathrm{b}}$ & $89.1 \times 10^{-3}[\mathrm{~mm}]$ \\
\hline
\end{tabular}

approach, see the discussion in [12], and in particular the peak stress $\sigma_{\mathrm{c}}^{\mathrm{pf}}$ and strain $\epsilon_{\mathrm{c}}^{\mathrm{pf}}$, with

$$
\sigma_{\mathrm{c}}^{\mathrm{pf}}=\frac{9}{16} \sqrt{\frac{G_{\mathrm{c}} E}{6 l^{\mathrm{pf}}}}=1.42 \mathrm{GPa} \text { and } \epsilon_{\mathrm{c}}^{\mathrm{pf}}=\sqrt{\frac{G_{\mathrm{c}}}{6 l^{\mathrm{pf}} E}}=0.012 .
$$

To this end, the power damage law (41) is still used and the value of the exponent $\beta$ is fixed to a small value while a high value of $\alpha$ is chosen in order to ensure a fast stress decrease followed by a long tail, which characterise the one-dimensional homogeneous phase field response. The two remaining parameters $\kappa_{\mathrm{i}}$ and $\kappa_{\mathrm{c}}$ are then computed in order to ensure that the stress peak is close to the values given by (79). The chosen material damage parameters are summarised in Table III and the corresponding stress-strain response is compared to the one-dimensional homogeneous phase field response in Fig. 14(a).

The non-local length is now calibrated using the previously described one-dimensional simulations with localisation, see Section 3, with the aim of recovering the same fracture energy $G_{\mathrm{c}}$ after localisation than the phase field model input. The resulting non-local length is evaluated to be $l_{\mathrm{c}}=16.5 \times 10^{-3} \mathrm{~mm}$. The corresponding one-dimensional stress response -with localisationof the non-local damage model is represented on Fig. 14(b). The fracture energy after localisation onset -which corresponds to a damage value $D_{\text {soft }}=0.44$ as obtained from (62)- in terms of the crack damage insertion is shown on Fig. 14(c). Clearly the critical energy release rate evaluated at softening onset is close to $G_{\mathrm{c}}=2700 \mathrm{~J} / \mathrm{m}^{2}$. Moreover, it can be seen in Fig. 14(d) that a comparable damage diffusion between the non-local damage model and the phase field solution is recovered. In this figure, the damage profile obtained at failure is compared with the idealised damage distribution of the phase field method, which reads [12]

$$
D^{\mathrm{pf}}(x)=e^{-|x| / 2 l^{\mathrm{pf}}} .
$$




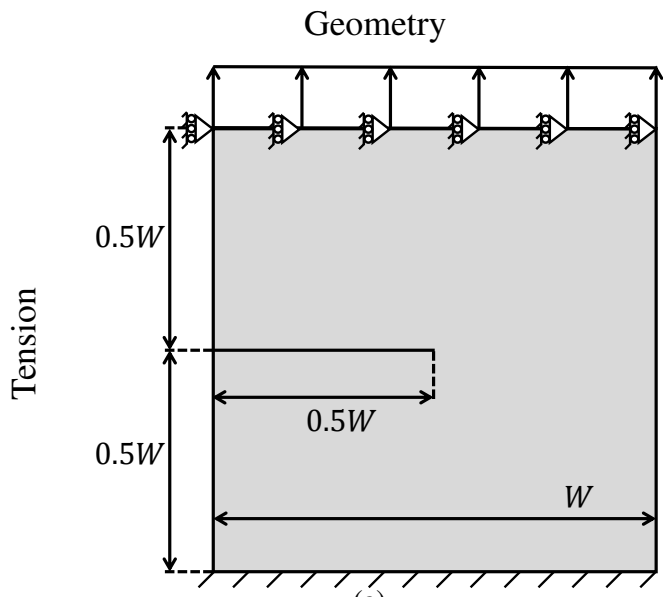

(a)

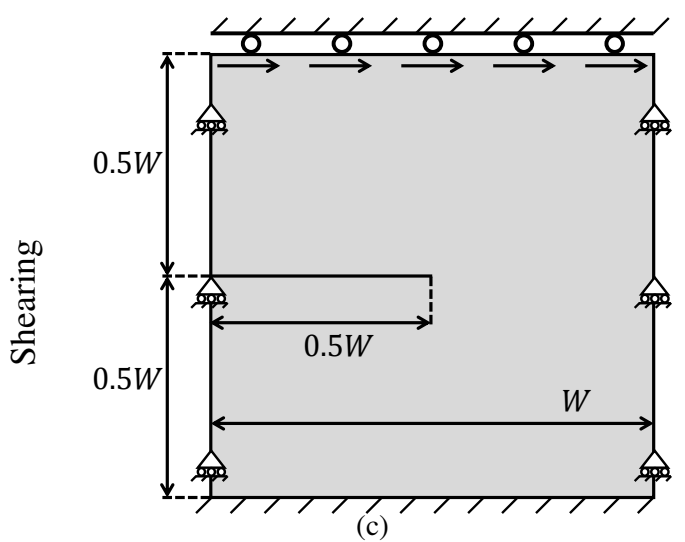

Mesh

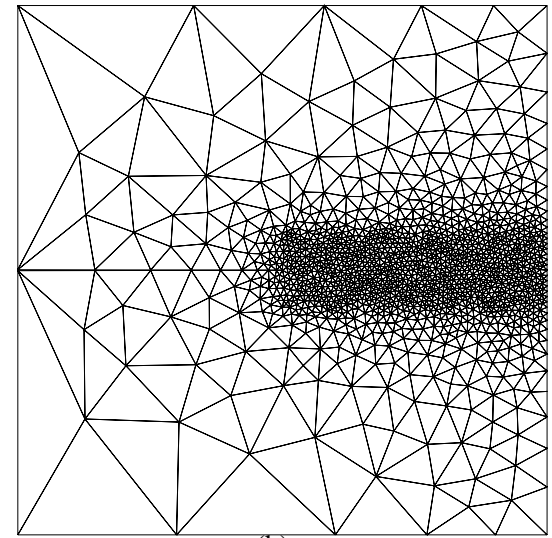

(b)

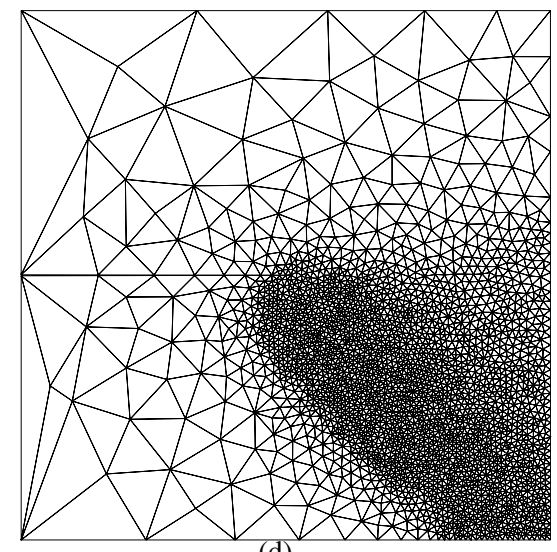

(d)

Figure 15. The single edge notched specimen geometries (left) of width $W=1 \mathrm{~mm}$ with their boundary conditions and the corresponding meshes (right) for the tensile (a-b) and shear cases (c-d).

Apart from the non-zero homogeneous damage value of the non-local model, both distributions exhibit a similar damage spread.

For the damage to crack transition parameters, the insertion effective stress is chosen so that the crack initiation appears around a damage value $D_{\mathrm{c}}=0.85$. The cohesive band thickness $h_{\mathrm{b}}$ is still computed as determined in Section 3 and is equal to $h_{\mathrm{b}}=5.4 l_{\mathrm{c}}$.

5.2.2. Tensile tests The tensile boundary conditions applied on the plate of width $W=1 \mathrm{~mm}$ are represented on Fig. 15(a). A prescribed vertical displacement is applied on the top edge while lateral edges remain traction-free. Both bottom and top edges are constrained along the horizontal direction. The specimen is meshed with 2779 quadratic triangular elements as shown on Fig. 15(b). Small elements, with a characteristic size of $0.01 \mathrm{~mm}$ are used along the expected crack path while coarser ones are used away from it. The system is solved using the implicit scheme detailed in Section 4.2.1 completed with a path-following method due to possible snap-back. 


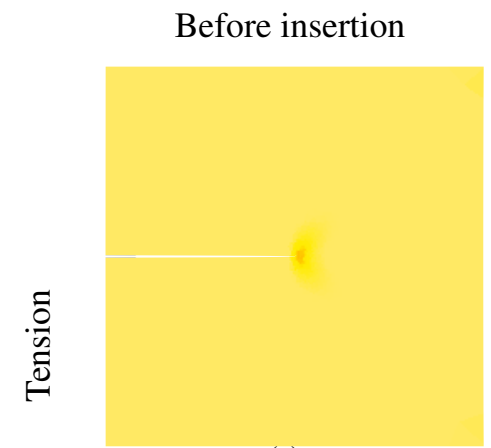

(a)

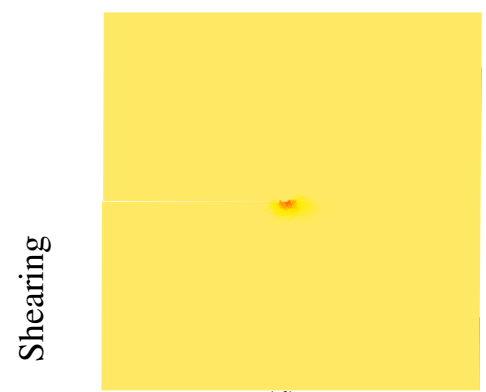

(d)
Non-local only

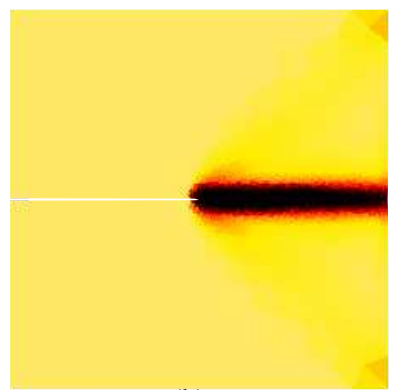

(b)

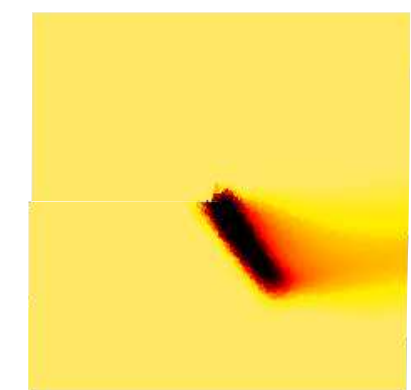

(e)
$\mathrm{CDM} / \mathrm{CBM}$

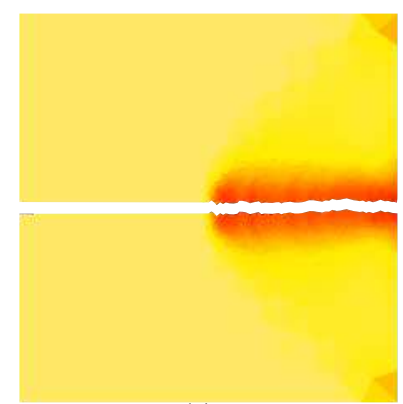

(c)

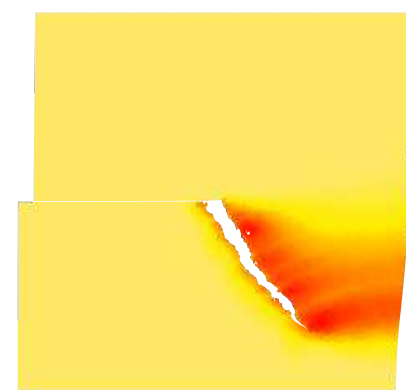

(f)

$D^{0}$ 0.5

Figure 16. Damage field (a and d) before crack insertion and at material failure for (b and e) the pure nonlocal case and (c and f) the CDM/CBM frameworks. Displacements have been magnified for the hybrid $\mathrm{CDM} / \mathrm{CBM}$ in order to visualise the crack path. The distributions are reported for the tensile (first row) and shear (second row) tests.

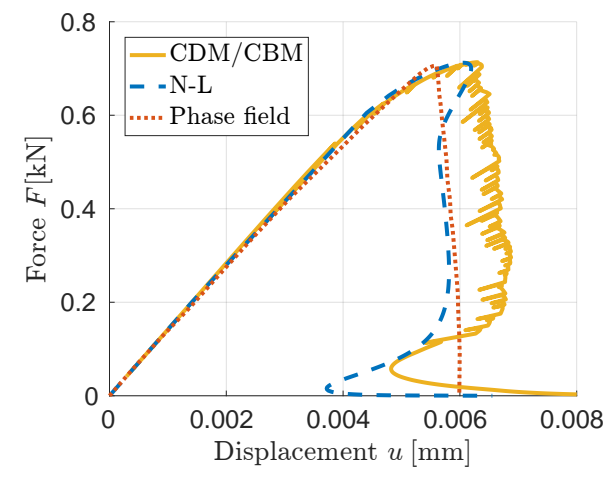

(a)

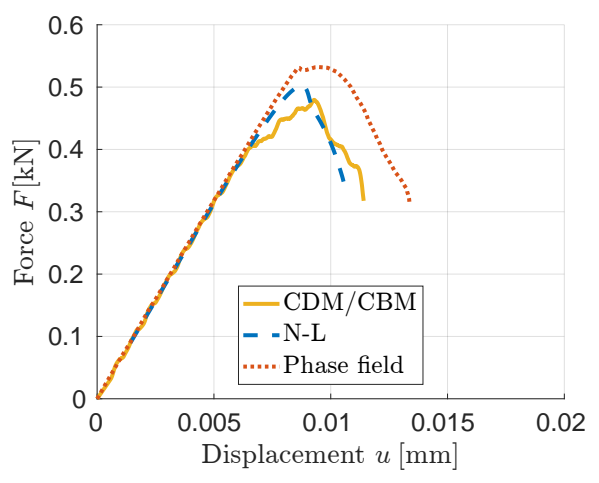

(b)

Figure 17. Loading force (for a $1 \mathrm{~mm}$-thick plate) $v s$. displacement evolution for the pure non-local, the hybrid CDM/CBM framework, and the phase field framework for (a) the tensile test and (b) the shear test.

The damage field before first crack insertion is developing at the crack tip, as shown on Fig. 16(a) and is then growing along the symmetry plane. The distributions obtained at material failure for the pure non-local model and the hybrid CDM/CBM framework are shown respectively on Fig. 16(b) 
and 16(c). The close-to-one damage band obtained with the non-local model is replaced by a crack for the hybrid CDM/CBM framework which is surrounded by lower damage values. The force evolution for both models is now compared on Fig. 17(a) with the results reported for the phase field method in [59]. Comparable results are obtained with the three methods: failure appears for the same peak load although it is slightly delayed for the non-local model and the hybrid CDM/CBM framework. For this application the pure non-local simulation exhibits a stronger snap-back than the hybrid CDM/CBM framework because of the spreading of the highly damage zone arising with the former method.

5.2.3. Shear tests Shear boundary conditions are now applied on the previous specimen as illustrated in Fig. 15(a): vertical displacement are constrained along all edges while a horizontal one is applied on the top one. The mesh, represented on Fig. 15(b), consists of 5362 quadratic triangles. Small elements (around $0.01 \mathrm{~mm}$ characteristic size) are located along a $45^{\circ}$-line along which the crack is assumed to propagate. The zone of refinement is wide enough in order to correctly describe the damage diffusion and to avoid any artificial guidance of the crack. The hybrid CDM/CBM system is solved using the coupled explicit-implicit integration scheme detailed in Section 4.2.2.

Figure 16(d) illustrates the damage distribution developing below the crack tip before first crack insertion. The distributions obtained for the same remaining force as the reported phase field results [59] are shown on Fig. 16(e) and 16(f) for respectively the pure non-local model and the hybrid CDM/CBM framework. The close-to-one damage band obtained with the non-local model is replaced by a crack kinking before following a $45^{\circ}$-angle for the hybrid CDM/CBM framework. Note that because the hybrid CDM/CBM simulations have been conducted with a dynamic explicit integration, some single dust elements arise during the crack opening and have been removed for visualisation purpose. The force $v s$. displacement histories of both models are compared on Fig. 17(b) with the phase field predictions reported in [59]. Note that because the hybrid CDM/CBM simulations have been conducted with a dynamic explicit integration, the resulting numerical oscillations have been filtered for visualisation purpose. The reached peak load is about $10 \%$ lower when using non-local CDM although the the softening strain is the same than with the phase-field model. However the peak stress of the phase field simulations is sensitive to the associated length scale $l^{\mathrm{pf}}$.

\subsection{Compact tension specimen test}

This test was studied experimentally and using the non-local CDM in [60]. The Compact Tension Specimen (CTS) tests were carried out on short glass-fiber-reinforced polypropylene. The geometry is shown in Fig. 18(a) with the dimensions $W=50 \mathrm{~mm}, a_{\mathrm{n}}=10 \mathrm{~mm}$ and the thickness $t=3.8 \mathrm{~mm}$.

The specimen is meshed with 5099 10-node 3D tetrahedral elements and partitioned between 16 processors as shown in Fig. 18(b). Quadratic shape functions are use to approximate both displacement and the non-local fields. Due to the symmetry of the system, only one half of the thickness is effectively modelled. A finer mesh is used near the expected crack path while a coarser one is used in other mesh regions. To apply the loading, loading pins are modelled with quarters of cylinder of higher stiffness $(E=30 \mathrm{GPa})$. The displacements of the pin axes are controlled in the vertical direction and constrained in the other ones, while axes rotation is left free. The 


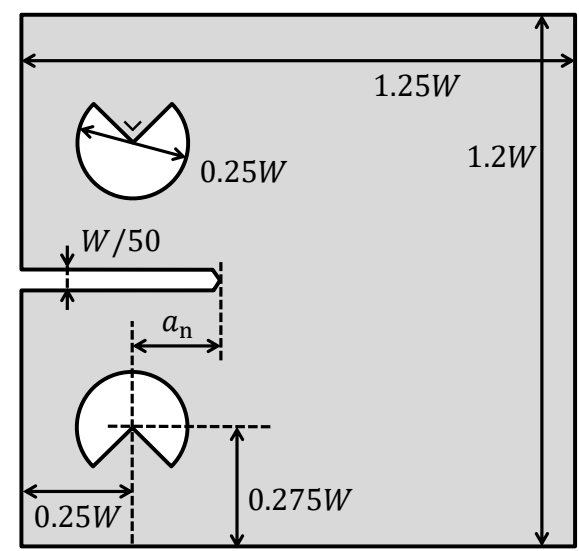

(a)

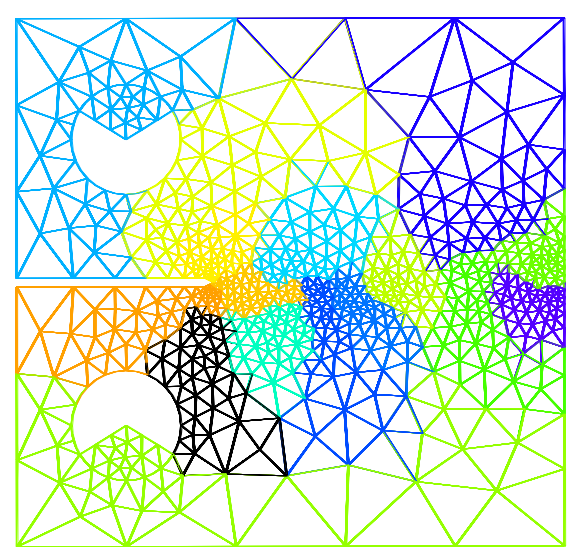

(b)

Figure 18. (a) The Compact-Tension Specimen geometry with $W=50 \mathrm{~mm}, a_{\mathrm{n}}=10 \mathrm{~mm}$ and $t=3,8 \mathrm{~mm}$; and (b) its partitioned mesh.

material properties are reported in Table I. The system is solved using the coupled explicit-implicit integration scheme detailed in Section 4.2.2.

The hybrid CDM/CBM framework is applied on the CTS in plane stress and compared with the experimental results [60], the CDM/CZM, and with the pure non-local model. The sample is also simulated in plane strain state using the three frameworks. The set of damage to crack transition parameters is given in Table II.

The loading force with respect to the displacements between both pins (evaluate for the full thickness), is represented in Fig. 19(a) in the plane stress state for the three different frameworks and for the experiment results [60] ${ }^{\ddagger}$. If numerical and experimental results are globally consistent, the CBM shows a better agreement with the experimental results than the other frameworks. A small discrepancy is observed from the beginning between the numerical results and the experimental ones, which is not due to the numerical framework, but to the finite element model itself since it is present even in the elastic part of the simulation. Indeed, the loading pins introduce a spurious compliance as there are not perfectly rigid; They should be replaced by rigid springs in compression without tension stiffness. Before crack insertion, no difference is observed between the frameworks as expected. After the crack is insert for both the CDM/CBM framework and the CDM/CZM framework, the curve predicted by the non-local model keeps increasing, resulting in an higher peak value. Then, the force quickly drops, due to the high damage spreading within the specimen. For the hybrid frameworks, both models allow a transition without unacceptable loss of energy. However, a much better agreement is obtained with the CDM/CBM than the CDM/CZM. Indeed, taking advantage of the constant value of the band thickness, the CBM does not suffer from the variation of damage value at which crack insertions occur. A similar analysis can be carried out in the plane strain state shown in Fig. 19(b).

\footnotetext{
¥The experimental results from [60] have been translated in order to pass by the origin.
} 


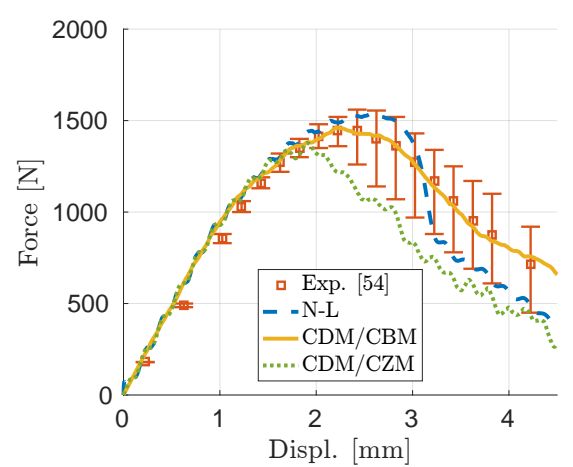

(a)

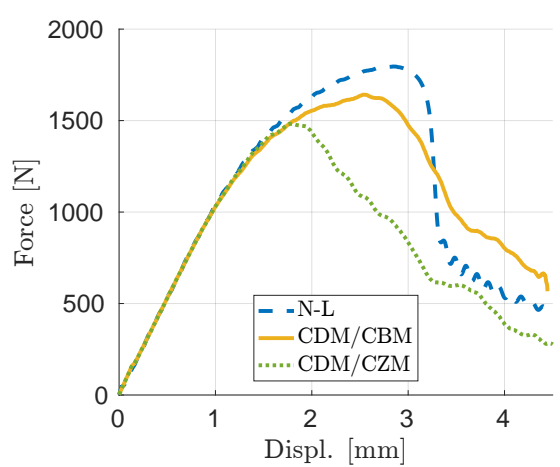

(b)

Figure 19. Loading force vs. pin displacement evolutions with the hybrid CDM/CBM framework, the $\mathrm{CDM} / \mathrm{CZM}$, the pure non-local, and the experimental measurements in (a) plane stress state and (b) in plane strain state.

The corresponding damage fields in the plate stress configuration are presented in the deformed configuration in Fig. 20(a) to 20(f) for a pin displacement $d=2 \mathrm{~mm}$. At this point, all models predict similar results. A crack has just started to propagate in the hybrid frameworks while damage is slightly more developed for the non-local model. For $d=4 \mathrm{~mm}$ in Fig. 21(a) to 21(f), the simulation involving only the non-local model suffers from high element distortions and spurious damage spreads from either side of the physical crack surface as no limiting mechanism was introduced. This non-physical spread reaches the free border of the initial notch. This problem is avoided with the hybrid frameworks where damage development is replaced by a crack propagation. In this case, the damage zone thickness remains constant along the crack path.

\section{CONCLUSIONS}

In this work, a cohesive band model was derived and introduced inside a damage to crack transition framework within a Discontinuous Galerkin approach.

A non-local damage model is used in the early stages of failure process. Its purpose is to simulate the diffuse degradation process without suffering a mesh-dependency. However, large distortions appear inside the most damaged elements, which induce spurious damage spreading and numerical problems.

To overcome this issue, a discontinuity is inserted through a cohesive band to simulate the last failure stage. It consists in a cohesive model with a finite thickness along which the stress is assumed to be uniform. The strains in this band are reconstructed by enhancing the neighbouring bulk strain by the cohesive jump. From this strain tensor, the bulk material damage constitutive law is used in its local way to compute the corresponding traction forces. By this way, triaxiality effects are naturally included inside the TSL of the CBM.

The numerical thickness of the CBM is not a new material parameter. One-dimensional setups are semi-analytically solved to compute the thickness which ensures the energetic consistency. It 


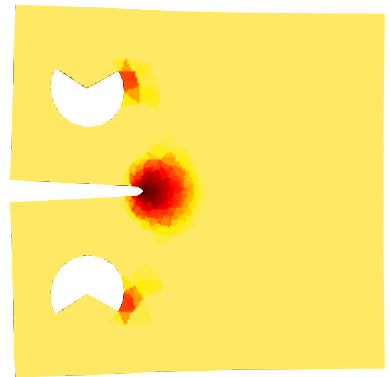

(a) Non-local

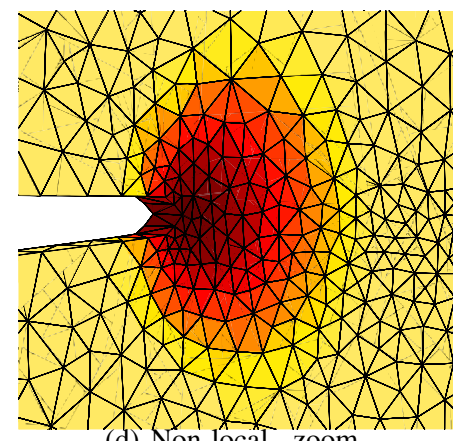

(d) Non-local - zoom

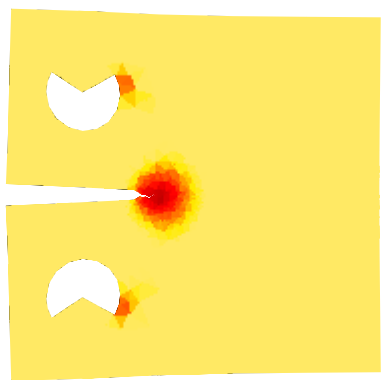

(b) $\mathrm{CDM} / \mathrm{CBM}$

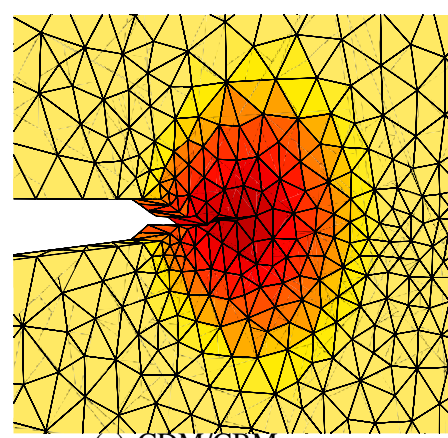

(e) CDM/CBM- zoom

0.5

$D^{0}$

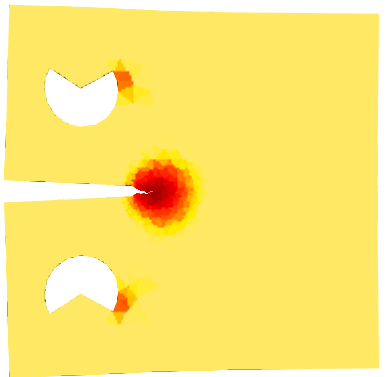

(c) $\mathrm{CDM} / \mathrm{CZM}$

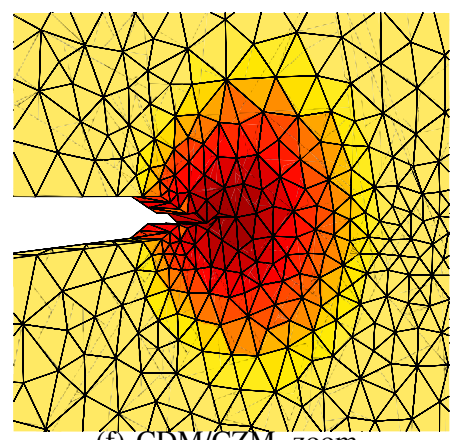

(f) CDM/CZM- zoom

Figure 20. Damage field at pin displacement $d=2 \mathrm{~mm}$ in the whole sample (top) and zoomed on the process zone (bottom) for the non-local model (left), the hybrid CDM/CBM (center) and the hybrid CDM/CZM (right).

appears that the thickness is independent of the damage model parameters and proportional to the non-local characteristic length as long as the crack is inserted during the softening stage.

This framework is then applied to 2D and 3D simulations using a simple crack insertion criterion based on effective stress. The CDM/CBM is implemented in parallel in an efficient way thanks to the DG framework. Indeed, the presence from the beginning of interfaces due to the DG formalism allows easy in-the-fly insertion of interface elements and thus avoids the usual issues of the CZM. The triaxiality sensitivity of the model is explicitly shown on a $2 \mathrm{D}$ plate bi-axialy loaded. The method is validated with the experimental results of a CTS test and is compared with a classical cohesive zone model, and with a pure non-local damage model. The results show that a better agreement is obtained during the whole failure process with the hybrid CDM/CBM than with the two others numerical models, i.e. than with the pure the non-local damage model and than with the hybrid CDM/CZM framework. Finally it has been shown that, by a proper identification of the material parameters of the underlying non-local damage model, the hybrid CDM/CBM can reproduce the prediction of phase field simulations.

This work is currently limited to elastic behaviour and small deformation setting. The next development step is to extend this framework to ductile failure for which the CBM might be promising since it allows to include triaxiality effects in the crack propagation process, which is mandatory for accurate ductile simulations. 


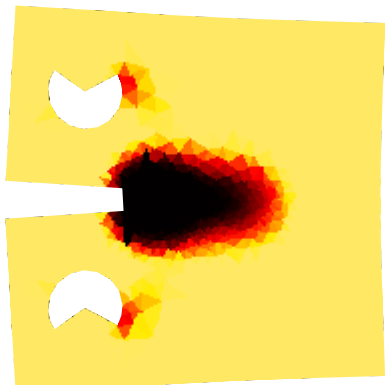

(a) Non-local

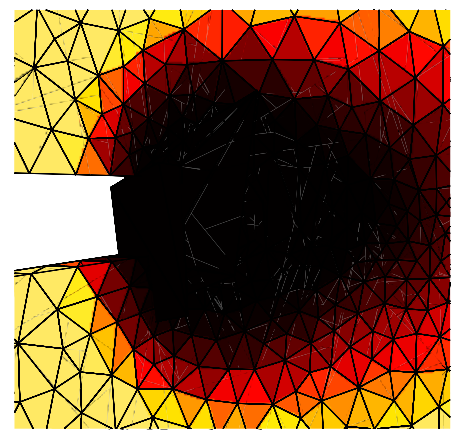

(d) Non-local - zoom

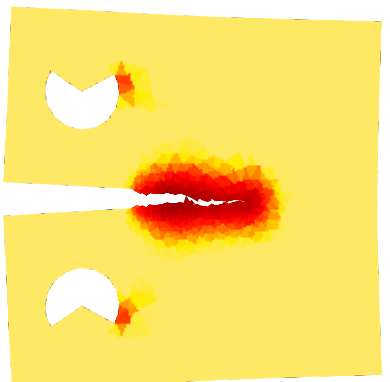

(b) $\mathrm{CDM} / \mathrm{CBM}$

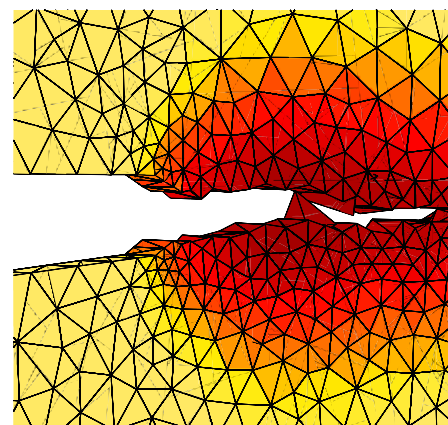

(e) CDM/CBM- zoom

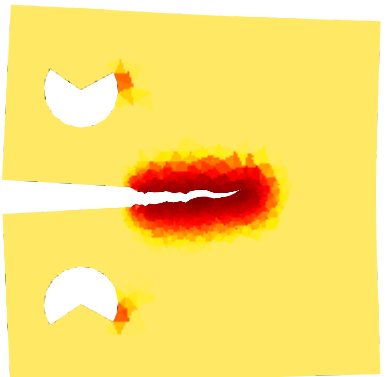

(c) $\mathrm{CDM} / \mathrm{CZM}$

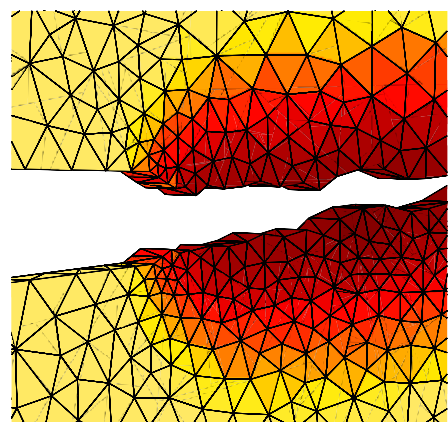

(f) CDM/CZM- zoom $D^{0}$ 0.5

Figure 21. Damage field at pin displacement $d=4 \mathrm{~mm}$ in the whole sample (top) and zoomed on the process zone (bottom) for the non-local model (left), the hybrid CDM/CBM (center) and the hybrid CDM/CZM (right).

\section{ACKNOWLEDGMENT}

The research has been funded by the Walloon Region under the agreement no.7581-MRIPF in the context of the 16th MECATECH call.

\section{A. LINEARISATION OF THE ELASTIC DAMAGE MODEL}

One has from Eq. (35):

$$
\delta \sigma_{i j}=\frac{\partial \sigma_{i j}}{\partial \epsilon_{k l}} \delta \epsilon_{k l}+\frac{\partial \sigma_{i j}}{\partial \tilde{e}} \delta \tilde{e} .
$$

Depending if the applied damage model is local or non-local, the derivatives $\frac{\partial \boldsymbol{\sigma}}{\partial \boldsymbol{\epsilon}}$ and $\frac{\partial \boldsymbol{\sigma}}{\partial \tilde{e}}$ have different expressions. For the implicit non-local damage model, one has

$$
\delta \sigma_{i j}=\underbrace{\left[(1-D) \mathcal{H}_{i j k l}\right]}_{\mathcal{C}_{u u}} \delta \epsilon_{k l}+\underbrace{\left[-\hat{\sigma}_{i j} \frac{\partial D}{\partial \tilde{e}}\right]}_{\mathcal{C}_{u \tilde{e}}} \delta \tilde{e},
$$


while for the local damage model, applied in the cohesive band

$$
\delta \sigma_{i j}=\underbrace{\left[(1-D) \mathcal{H}_{i j k l}-\hat{\sigma}_{i j} \frac{\partial D}{\partial \epsilon_{k l}}\right]}_{\mathcal{C}_{\text {loc }}^{\text {loc }}} \delta \epsilon_{k l} .
$$

Concerning the local value $e$, the linearisation of Eq. (37) gives

$$
\delta e=\frac{\partial e}{\partial \epsilon_{i j}} \delta \epsilon_{i j}=\underbrace{\left[\sum_{k}^{+} \epsilon_{k} \frac{v_{i}^{k} v_{j}^{k}}{e}\right]}_{\mathcal{C}_{e u}} \delta \epsilon_{i j},
$$

where $\epsilon_{k}$ is a eigenvalue of $\boldsymbol{\epsilon}, \boldsymbol{v}^{k}$ is the associated eigenvector, and where the sum $\sum_{k}^{+}$carries only on positive eigenvalues.

\section{B. COMPUTATION AND LINEARISATION OF THE COHESIVE BAND MODEL STRESS TENSOR}

The band deformation gradient is computed by applying the Eq. (29) on the corresponding side of the interface, using the corresponding deformation gradient $F_{i J}$, the jump displacement $\llbracket u_{i} \rrbracket$ and the gradient of the jump displacement $\nabla_{J} \llbracket u_{i} \rrbracket$ :

$$
\begin{aligned}
F_{\mathrm{b}_{i J}}=F_{i J} & +n_{\mathrm{b}_{i}} n_{\mathrm{b}_{k}}\left[\left(F_{\mathrm{c}_{k L}}-I_{k L}\right) \frac{\delta_{\mathrm{n}}}{\delta_{\mathrm{n}}^{\text {max }}}-\left(F_{k L}-I_{k L}\right)\right] N_{\mathrm{b}_{L}} N_{\mathrm{b}_{J}} \\
& +t_{\mathrm{b}_{i}} t_{\mathrm{b}_{k}}\left[\left(F_{\mathrm{c}_{k L}}-I_{k L}\right) \frac{\delta_{\mathrm{t}}}{\delta_{\mathrm{t}}^{\text {max }}}-\left(F_{k L}-I_{k L}\right)\right] N_{\mathrm{b}_{L}} N_{\mathrm{b}_{J}} \\
& +s_{\mathrm{b}_{i}} s_{\mathrm{b}_{k}}\left[\left(F_{\mathrm{c}_{k L}}-I_{k L}\right) \frac{\delta_{\mathrm{s}}}{\delta_{\mathrm{s}}^{\text {max }}}-\left(F_{k L}-I_{k L}\right)\right] N_{\mathrm{b}_{L}} N_{\mathrm{b}_{J}} \\
& +\frac{\llbracket u_{i} \rrbracket N_{\mathrm{b}_{J}}}{h_{\mathrm{b}}}+\frac{1}{2} \frac{\partial \llbracket u_{i} \rrbracket}{\partial X_{J}} .
\end{aligned}
$$

In this expression, we have omitted the exponent \pm related to the neighbouring elements. The deformation gradient $F_{i J}$ and the jump displacement $\llbracket u_{i} \rrbracket$ are computed with the shape function of the volume elements while the computation of $\nabla_{J} \llbracket u_{i} \rrbracket$ involves the shape functions of the interface elements only (because a node outside the interface does not have to influence this value in order to satisfy the condition (26)). As jump values and their gradients are not equal to zero before crack insertion due to DG formalism, initial jump values (and their gradients) at crack insertion are deducted from the effective values used in (85). The terms $\delta_{\mathrm{n}} / \delta_{\mathrm{n}}^{\max }, \delta_{\mathrm{t}} / \delta_{\mathrm{t}}^{\max }$, and $\delta_{\mathrm{s}} / \delta_{\mathrm{s}}^{\max }$ introduce a linear unloading in case of crack closing in each direction of the local basis $\left(n_{\mathrm{b}}, \boldsymbol{t}_{\mathrm{b}}, \boldsymbol{s}_{\mathrm{b}}\right)$. $\delta_{\mathrm{n}}^{\max }, \delta_{\mathrm{t}}^{\max }$ and $\delta_{\mathrm{s}}^{\max }$ are the maximal values reached during crack opening, following

$$
\begin{aligned}
& \delta_{\mathrm{n}}^{\max }=\max \left(\delta_{\mathrm{n}}(\tau), \tau \in[0, t]\right) ; \\
& \delta_{\mathrm{t}}^{\max }=\max \left(\delta_{\mathrm{t}}(\tau), \tau \in[0, t]\right) ; \\
& \delta_{\mathrm{s}}^{\text {max }}=\max \left(\delta_{\mathrm{s}}(\tau), \tau \in[0, t]\right) .
\end{aligned}
$$


To avoid an indetermination and infinite stiffness in case of unloading just after crack insertion, an offset $\delta_{0}$ is added to the effective openings $\delta_{\mathrm{n}}, \delta_{\mathrm{t}}$, and $\delta_{\mathrm{s}}$ at crack insertion. Practically, the effective opening in the different directions read

$$
\delta_{\mathrm{n}}=\llbracket \boldsymbol{u} \rrbracket \cdot \boldsymbol{n}_{\mathrm{b}}+\delta_{0}, \delta_{\mathrm{t}}=\llbracket \boldsymbol{u} \rrbracket \cdot \boldsymbol{t}_{\mathrm{b}}+\delta_{0}, \text { and } \delta_{\mathrm{s}}=\llbracket \boldsymbol{u} \rrbracket \cdot \boldsymbol{s}_{\mathrm{b}}+\delta_{0}
$$

The value of $\delta_{0}$ is fixed around $10^{-3} h_{\mathrm{b}}[\mathrm{m}]$, to be small enough to avoid spurious jump offset without hurting numerical convergence or stability.

The derivatives of the band deformation gradient $\mathbf{F}_{\mathrm{b}}$ (85) with respect to the bulk deformation gradient $\mathbf{F}$ read

$$
\frac{\partial F_{\mathrm{b}_{i J}}}{\partial F_{k L}}=\frac{\partial}{\partial F_{k L}}\left[F_{i J}-F_{i M} N_{\mathrm{b}_{M}} N_{\mathrm{b}_{J}}\right]=\delta_{i k} \delta_{J L}-\delta_{i k} N_{\mathrm{b}_{J}} N_{\mathrm{b}_{L}}
$$

and, with respect to the jump displacement at the interface read

$$
\begin{aligned}
\frac{\partial F_{\mathrm{b}_{i J}}}{\partial \llbracket u_{k} \rrbracket}= & n_{\mathrm{b}_{i}} n_{\mathrm{b}_{m}}\left[F_{\mathrm{c}_{m L}}-I_{m L}\right] N_{\mathrm{b}_{L}} N_{\mathrm{b}_{J}} \frac{n_{\mathrm{b}_{k}}}{\delta_{\mathrm{n}^{\text {max }}}} \mathbf{1}_{\left(<\delta_{\mathrm{n}}^{\text {max }}\right)}\left(\delta_{\mathrm{n}}\right) \\
& +t_{\mathrm{b}_{i}} t_{\mathrm{b}_{m}}\left[F_{\mathrm{c}_{m L}}-I_{m L}\right] N_{\mathrm{b}_{L}} N_{\mathrm{b}_{J}} \frac{t_{\mathrm{b}_{k}}}{\delta_{\mathrm{t}^{\text {max }}}^{\text {max }}} \mathbf{1}_{\left(<\delta_{\mathrm{t}}^{\text {max }}\right)}\left(\delta_{\mathrm{t}}\right) \\
& +s_{\mathrm{b}_{i}} s_{\mathrm{b}_{m}}\left[F_{\mathrm{c}_{m L}}-I_{m L}\right] N_{\mathrm{b}_{L}} N_{\mathrm{b}_{J}} \frac{s_{\mathrm{b}_{k}}}{\delta_{\mathrm{s}}^{\text {max }}} \mathbf{1}_{\left(<\delta_{\mathrm{s}}^{\text {max }}\right)}\left(\delta_{\mathrm{s}}\right) \\
& +\frac{N_{\mathrm{b}_{J}}}{h_{\mathrm{b}}} \delta_{i k},
\end{aligned}
$$

where the function $\mathbf{1}_{y}(x)$ is equal to 1 if the argument $x$ satisfies the condition $y$, and to 0 otherwise. The derivative with respect to the gradient of the displacement jump at the interface reads

$$
\frac{\partial F_{\mathrm{b}_{i J}}}{\partial \nabla_{L} \llbracket u_{k} \rrbracket}=\frac{1}{2} \delta_{i k} \delta_{J L}
$$

Then, the stress tensor $\boldsymbol{\sigma}_{\mathrm{b}}$ is computed using Eq. (42) as damage law evolution, leading to

$$
\left\langle\boldsymbol{\sigma}_{\mathrm{b}}\right\rangle=\frac{\boldsymbol{\sigma}_{\mathrm{b}}^{+}\left(\boldsymbol{\epsilon}^{+}, \boldsymbol{Z}^{+}\right)+\boldsymbol{\sigma}_{\mathrm{b}}^{-}\left(\boldsymbol{\epsilon}^{-}, \boldsymbol{Z}^{-}\right)}{2}
$$

Finally, the material tangent tensor of the cohesive band model can be obtained by linearisation of the band stress tensor:

$$
\begin{aligned}
\delta\left\langle\sigma_{\mathrm{b}_{i j}}\right\rangle= & \frac{\partial\left\langle\sigma_{\mathrm{b}_{i j}}\right\rangle}{\partial F_{m n}^{+}} \delta F_{m n}^{+}+\frac{\partial\left\langle\sigma_{\mathrm{b}_{i j}}\right\rangle}{\partial F_{m n}^{-}} \delta F_{m n}^{-}+\frac{\partial\left\langle\sigma_{\mathrm{b}_{i j}}\right\rangle}{\partial \llbracket u_{m} \rrbracket} \delta \llbracket u_{m} \rrbracket+\frac{\partial\left\langle\sigma_{\mathrm{b}_{i j}}\right\rangle}{\partial \nabla_{n} \llbracket u_{m} \rrbracket} \delta \nabla_{n} \llbracket u_{m} \rrbracket \\
= & \frac{1}{2} \frac{\partial \sigma_{\mathrm{b}_{i j}}^{+}}{\partial F_{\mathrm{b}_{k l}}^{+}} \frac{\partial F_{\mathrm{b}_{k l}}^{+}}{\partial F_{m n}^{+}} \delta F_{m n}^{+}+\frac{1}{2} \frac{\partial \sigma_{\mathrm{b}_{i j}}^{-}}{\partial F_{\mathrm{b}_{k l}}^{-}} \frac{\partial F_{\mathrm{b}_{k l}}^{-}}{\partial F_{m n}^{-}} \delta F_{m n}^{-} \\
& +\frac{1}{2}\left(\frac{\partial \sigma_{\mathrm{b}_{i j}}^{+}}{\partial F_{\mathrm{b}_{k l}}^{+}} \frac{\partial F_{\mathrm{b}_{k l}}^{+}}{\partial \llbracket u_{m} \rrbracket}+\frac{\partial \sigma_{\mathrm{b}_{i j}}^{-}}{\partial F_{\mathrm{b}_{k l}}^{-}} \frac{\partial F_{\mathrm{b}_{k l}}^{-}}{\partial \llbracket u_{m} \rrbracket}\right) \delta \llbracket u_{m} \rrbracket \\
& +\frac{1}{2}\left(\frac{\partial \sigma_{\mathrm{b}_{i j}}^{+}}{\partial F_{\mathrm{b}_{k l}}^{+}} \frac{\partial F_{\mathrm{b}_{k l}}^{+}}{\partial \llbracket u_{m} \rrbracket}+\frac{\partial \sigma_{\mathrm{b}_{i j}}^{-}}{\partial F_{\mathrm{b}_{k l}}^{-}} \frac{\partial F_{\mathrm{b}_{k l}}^{-}}{\partial \nabla_{n} \llbracket u_{m} \rrbracket}\right) \delta \nabla_{n} \llbracket u_{m} \rrbracket .
\end{aligned}
$$


By introducing

$$
\mathcal{C}_{\boldsymbol{u} \mathbf{F}_{i j m n}}^{\mathrm{b} \pm}=\mathcal{C}_{\boldsymbol{u} \boldsymbol{u}_{i j k l} \operatorname{loc}_{i j l}}^{\text {lo }} \frac{\partial F_{\mathrm{b}_{k l}}^{ \pm}}{\partial F_{m n}^{ \pm}} ; \quad \mathcal{C}_{\boldsymbol{u} \llbracket \boldsymbol{u} \rrbracket_{i j m}}^{\mathrm{b}}=\mathcal{C}_{\boldsymbol{u} \boldsymbol{u}_{i j k l}}^{\mathrm{loc} \pm} \frac{\partial F_{\mathrm{b}_{k l}}^{ \pm}}{\partial \llbracket u_{m} \rrbracket} ; \quad \mathcal{C}_{\boldsymbol{u} \llbracket \boldsymbol{u} \rrbracket_{i j m}}^{\mathrm{b} \pm}=\mathcal{C}_{\boldsymbol{u} \boldsymbol{u}_{i j k l}}^{\text {loc }} \frac{\partial F_{\mathrm{b}_{k l}}^{ \pm}}{\partial \nabla_{l} \llbracket u_{k} \rrbracket},
$$

with $\mathcal{C}_{\boldsymbol{u} u}^{\text {loc }}$ as the material tangent in its local form, the previous relation reads

$$
\delta\left\langle\sigma_{\mathrm{b}_{i j}}\right\rangle=\frac{1}{2} \mathcal{C}_{\boldsymbol{u} \mathbf{F}}^{\mathrm{b}+} \delta F_{m n}^{+}+\frac{1}{2} \mathcal{C}_{\boldsymbol{u} \mathbf{F}}^{\mathrm{b}-} \delta F_{m n}^{-}+\left\langle\mathcal{C}_{\boldsymbol{u} \llbracket \boldsymbol{b} \rrbracket}^{\mathrm{b}}\right\rangle \delta \llbracket u_{k} \rrbracket+\left\langle\mathcal{C}_{\boldsymbol{u} \boldsymbol{\nabla} \llbracket \boldsymbol{u} \rrbracket}^{\mathrm{b}}\right\rangle \delta \nabla_{l} \llbracket u_{k} \rrbracket
$$

\section{FORMULATION OF FINITE ELEMENT FORCES}

From the weak form (66), the nodal forces are derived by applying the discretisation (67). This leads to the following elementary nodal forces for the volume elements $B^{e}$ and the interface elements $\Gamma^{s}$.

- Inertial forces $\mathbf{M}^{e a b} \cdot \ddot{\boldsymbol{u}}^{b}$ :

$$
\delta \boldsymbol{u}^{a} \cdot \mathbf{M}^{e a b} \cdot \ddot{\boldsymbol{u}}^{b}=\int_{B^{e}} \rho \boldsymbol{w}_{\boldsymbol{u}} \cdot \ddot{\boldsymbol{u}} d V=\delta \boldsymbol{u}^{a} \cdot \int_{B^{e}} N^{a} \rho N^{b} d V \cdot \ddot{\boldsymbol{u}}^{b} ;
$$

- Internal forces $\boldsymbol{f}_{\boldsymbol{u} \text { int }}^{e}{ }^{a}$ :

$$
\delta \boldsymbol{u}^{a} \cdot \boldsymbol{f}_{\boldsymbol{u} i n t}^{e}{ }^{a}=\int_{B^{e}} \boldsymbol{\nabla} \boldsymbol{w}_{\boldsymbol{u}}: \boldsymbol{\sigma} d V=\delta \boldsymbol{u}^{a} \cdot \int_{B^{e}} \boldsymbol{\sigma} \cdot \boldsymbol{\nabla} N^{a} d V
$$

- External forces $\boldsymbol{f}_{e x t}^{e}{ }^{a}$ :

$$
\begin{aligned}
\delta \boldsymbol{u}^{a} \cdot \boldsymbol{f}_{\text {ext }}^{e a} & =\int_{B^{e}} \rho \boldsymbol{w}_{\boldsymbol{u}} \cdot \boldsymbol{b} d V+\int_{\Gamma_{N}^{s}} \boldsymbol{w}_{\boldsymbol{u}} \cdot \overline{\boldsymbol{t}} d S \\
& =\delta \boldsymbol{u}^{a} \cdot \int_{B^{e}} \rho N^{a} \boldsymbol{b} d V+\delta \boldsymbol{u}^{a} \cdot \int_{\Gamma_{N}} N^{a} \overline{\boldsymbol{t}} d S
\end{aligned}
$$

- Interface forces $\boldsymbol{f}_{\boldsymbol{u} \mathrm{I}}^{s}{ }^{a^{ \pm}}$

$$
\begin{aligned}
& \delta \boldsymbol{u}^{a^{ \pm}} \cdot \boldsymbol{f}_{\boldsymbol{u} \mathrm{I}}^{s a^{ \pm \pm}}=\int_{\Gamma_{\mathrm{CI}}^{s}} \llbracket \boldsymbol{w}_{\boldsymbol{u}} \rrbracket \cdot\left\langle\boldsymbol{\sigma}_{\mathrm{b}}\right\rangle \cdot \boldsymbol{n}_{\mathrm{b}} d S+\int_{\Gamma_{\mathrm{UI}}^{s}} \llbracket \boldsymbol{w}_{\boldsymbol{u}} \rrbracket \cdot\langle\boldsymbol{\sigma}\rangle \cdot \boldsymbol{n}_{\mathrm{b}} d S \\
& +\int_{\Gamma_{\mathrm{UI}}^{s}} \llbracket \boldsymbol{w}_{\boldsymbol{u}} \rrbracket \otimes \boldsymbol{n}_{\mathrm{b}}:\left\langle\frac{\beta_{s}}{h_{s}} \mathcal{H}\right\rangle: \llbracket \boldsymbol{u} \rrbracket \otimes \boldsymbol{n}_{\mathrm{b}} d S \\
& +\int_{\Gamma_{\mathrm{UI}}^{s}}^{\mathrm{UI}} \llbracket \boldsymbol{u} \rrbracket \cdot\left\langle\mathcal{H}: \nabla \boldsymbol{w}_{\boldsymbol{u}}\right\rangle \cdot \boldsymbol{n}_{\mathrm{b}} d S \\
& =\delta \boldsymbol{u}^{a^{ \pm}} \cdot \int_{\Gamma_{\mathrm{CI}}^{s}}\left( \pm N^{a^{ \pm}}\right)\left\langle\boldsymbol{\sigma}_{\mathrm{b}}\right\rangle \cdot \boldsymbol{n}_{\mathrm{b}} d S+\delta \boldsymbol{u}^{a^{ \pm}} \cdot \int_{\Gamma_{\mathrm{UI}}^{s}}\left( \pm N^{a^{ \pm}}\right)\langle\boldsymbol{\sigma}\rangle \cdot \boldsymbol{n}_{\mathrm{b}} d S \\
& +\delta \boldsymbol{u}^{a^{ \pm}} \cdot \int_{\Gamma_{\mathrm{U}}^{s}}\left( \pm N^{a^{ \pm}}\right) \boldsymbol{n}_{\mathrm{b}} \cdot\left[\left\langle\frac{\beta_{s}}{h_{s}} \mathcal{H}\right\rangle: \llbracket N^{b} \boldsymbol{u}^{b} \rrbracket \otimes \boldsymbol{n}_{\mathrm{b}}\right] d S \\
& +\frac{1}{2} \delta \boldsymbol{u}^{a^{ \pm}} \cdot \int_{\Gamma_{\mathrm{UI}}^{s}} \llbracket N^{b} \boldsymbol{u}^{b} \rrbracket \cdot\left[\mathcal{H}^{ \pm}: \nabla N^{a^{ \pm}} \otimes \boldsymbol{n}_{\mathrm{b}}\right] d S
\end{aligned}
$$


- Internal non-local forces $f_{\tilde{e}}^{e}{ }^{a}{ }^{a}$ :

$$
\delta \tilde{e}^{a} f_{\tilde{e} \text { int }}^{e}{ }^{a}=\int_{B^{e}}\left(w_{\tilde{e}} \tilde{e}+l_{\mathrm{c}}^{2} \nabla w_{\tilde{e}} \cdot \nabla \tilde{e}\right) d V=\delta \tilde{e}^{a} \int_{B^{e}}\left(N^{a} N^{b} \tilde{e}^{b}+l_{\mathrm{c}}^{2} \boldsymbol{\nabla} N^{a} \cdot \nabla N^{b} \tilde{e}^{b}\right) d V
$$

- Internal local forces $f_{\text {eint }}^{e}{ }^{a}$ :

$$
\delta \tilde{e}^{a} f_{e i n t}^{e}{ }^{a}=\int_{B^{e}} w_{\tilde{e}} e d V=\delta \tilde{e}^{a} \int_{B^{e}} N^{a} e d V
$$

- Interface forces $f_{\tilde{e} \mathrm{I}}^{s a^{ \pm}}$

$$
\begin{aligned}
\delta \tilde{e}^{a^{ \pm}} f_{\tilde{e} \mathrm{I}}^{s} a^{ \pm}= & \int_{\Gamma_{\mathrm{UI}}^{s}} \llbracket w_{\tilde{e}} \rrbracket\left\langle l_{\mathrm{c}}^{2} \nabla \tilde{e}\right\rangle \cdot \boldsymbol{n}^{-} d S+\int_{\Gamma_{\mathrm{UI}}^{s}} \llbracket w_{\tilde{e}} \rrbracket \boldsymbol{n}_{\mathrm{b}}\left\langle\frac{\beta_{s}}{h_{s}} l_{\mathrm{c}}^{2}\right\rangle \cdot \boldsymbol{n}_{\mathrm{b}} \llbracket \tilde{e} \rrbracket d S \\
& +\int_{\Gamma_{\mathrm{UI}}^{s}} \llbracket \tilde{e} \rrbracket\left\langle l_{\mathrm{c}}^{2} \nabla w_{\tilde{e}}\right\rangle \cdot \boldsymbol{n}_{\mathrm{b}} d S \\
= & \delta \tilde{e}^{a^{ \pm}} \int_{\Gamma_{\mathrm{UI}}^{s}}\left( \pm N^{a^{ \pm}}\right)\left\langle l_{\mathrm{c}}^{2} \nabla N^{b} \tilde{e}^{b}\right\rangle \cdot \boldsymbol{n}^{-} d S \\
& +\delta \tilde{e}^{a^{ \pm}} \int_{\Gamma_{\mathrm{U}}^{s}}\left( \pm N^{a^{ \pm}}\right)\left\langle\frac{\beta_{s}}{h_{s}} l_{\mathrm{c}}^{2}\right\rangle \llbracket N^{b} \tilde{e}^{b} \rrbracket d S \\
& +\frac{1}{2} \delta \tilde{e}^{a^{ \pm}} \int_{\Gamma_{\mathrm{UI}}^{s}} \llbracket N^{b} \tilde{e}^{b} \rrbracket l_{\mathrm{c}}^{ \pm} \nabla^{a^{ \pm}} \cdot \boldsymbol{n}_{\mathrm{b}} d S .
\end{aligned}
$$

In this section, the superscripts $a, b, \ldots$ refer to the corresponding node $a$ of the considered element $e$. For interface elements $s$, the node is so-called $a^{+}$or $a^{-}$, depending to which element between $B^{e+}$ or $B^{e-}$ the considered degree of freedom belongs to. The computation of the spatial gradient of the jump displacement field involves the shape functions associated with interface elements only in order to respect condition (26). So-called $N_{s}^{a^{ \pm}}$, they are different from their volume counterparts noted $N^{a^{ \pm}}$. Besides, the displacement field in the nodal forces are the ones associated to volume elements. Therefore, the symmetrisation term introduces a contribution of all nodes of both elements in the interface term. Integration on the volume is performed using reduced quadrature rules, while interface elements use full integration to avoid spurious penetration mode [26]. Notice the proposed implementation does not duplicate the nodes between shared interfaces, but directly the degrees of freedom by taking advantage of the flexible DoF manager of Gmsh.

\section{FORMULATION OF FINITE ELEMENT STIFFNESS MATRICES}

The elementary stiffness matrices can be obtained by linearising the elementary forces of Eqs. (95) to (101). Those expressions involve material tangent matrices $\mathcal{C}_{\boldsymbol{u} \boldsymbol{u}_{i j k l}}, \mathcal{C}_{\boldsymbol{u} \tilde{e}_{i j}}$ and $\mathcal{C}_{e \boldsymbol{u}_{k i}}$ and tangent matrices related to the cohesive band $\mathcal{C}_{\boldsymbol{u} \llbracket \boldsymbol{u} \rrbracket_{i j k}}^{\mathrm{b}}, \mathcal{C}_{\boldsymbol{u} \nabla \llbracket \boldsymbol{u} \rrbracket_{i j k l}}$ and $\mathcal{C}_{\boldsymbol{u}}^{\mathrm{b}} \mathbf{F}_{i j k l}$. They are respectively detailed in Appendix A and B. Therefore, the different stiffness matrix contributions read

- Internal force stiffness matrices $K_{\boldsymbol{u} u \text { int }_{i j}}^{e}{ }_{i j}$ and $K_{\boldsymbol{u} e \tilde{\operatorname{int}}}^{e a b}{ }_{i}^{a}$ : 


$$
\begin{aligned}
K_{\boldsymbol{u u}_{\text {int }}{ }_{i k}}^{e b} & =\frac{\partial f_{\boldsymbol{u}_{\text {int }}^{e}}^{a}}{\partial u_{k}^{b}}=\int_{B^{e}} \nabla_{j} N^{a} \frac{\partial \sigma_{i j}}{\partial u_{k}^{b}} d V=\int_{B^{e}} \nabla_{j} N^{a} \mathcal{C}_{\boldsymbol{u} \boldsymbol{u}_{i j k l}} \nabla_{l} N^{b} d V, \\
K_{\boldsymbol{u} \tilde{e} \text { int }_{i}{ }_{i}}^{e b} & =\frac{\partial f_{\boldsymbol{u} \text { int }_{i}}^{e}}{\partial \tilde{e}^{b}}=\int_{B^{e}} \nabla_{j} N^{a} \frac{\partial \sigma_{i j}}{\partial \tilde{e}^{b}} d V=\int_{B^{e}} \nabla_{j} N^{a} \mathcal{C}_{\boldsymbol{u} \tilde{e}_{i j}} N^{b} d V
\end{aligned}
$$

- Interface force stiffness matrices $K_{u u \mathrm{I}_{i j}}^{s} \underset{a^{ \pm} b^{ \pm}}{ }$and $K_{\boldsymbol{u} \tilde{e} \mathrm{I}_{i}}^{s}{ }^{a^{ \pm} b^{ \pm}}$:

$$
\begin{aligned}
& K_{\boldsymbol{u} u \mathrm{I}_{i k}}^{s a^{ \pm} b^{ \pm}}=\frac{\partial f_{\boldsymbol{u} I_{i}}^{s} a^{ \pm}}{\partial u_{k}^{b^{ \pm}}} \\
& =\int_{\Gamma_{\mathrm{CI}}^{s}}\left( \pm N^{a^{ \pm}}\right)\left\langle\frac{\partial \sigma_{\mathrm{b}_{i j}}}{\partial u_{k}^{b^{ \pm}}}\right\rangle n_{\mathrm{b}_{j}} d S+\int_{\Gamma_{\mathrm{UI}}^{s}}\left( \pm N^{a^{ \pm}}\right)\left\langle\frac{\partial \sigma_{i j}}{\partial u_{k}^{b^{ \pm}}}\right\rangle n_{\mathrm{b} j} d S \\
& +\int_{\Gamma_{\mathrm{UI}}^{s}}\left( \pm N^{a^{ \pm}}\right) n_{\mathrm{b} j}\left\langle\frac{\beta_{s}}{h_{s}} \mathcal{H}_{i j k l}\right\rangle\left( \pm N^{b^{ \pm}}\right) n_{\mathrm{b} l} d S \\
& +\frac{1}{2} \int_{\Gamma_{\mathrm{UI}}^{s}}\left( \pm N^{b^{ \pm}}\right) \mathcal{H}_{i k j l}^{ \pm} \nabla_{j} N^{a^{ \pm}} n_{\mathrm{bl}} d S \\
& =\int_{\Gamma_{\mathrm{CI}}^{s}}\left( \pm N^{a^{ \pm}}\right)\left(\left\langle\mathcal{C}_{\boldsymbol{u} \llbracket \boldsymbol{u} \rrbracket_{i j k}}^{\mathrm{b}}\right\rangle\left( \pm N_{s}^{b^{ \pm}}\right)\right. \\
& \left.+\left\langle\mathcal{C}_{\boldsymbol{u} \nabla \llbracket \boldsymbol{u} \rrbracket_{i j k l}}^{\mathrm{b}}\right\rangle\left( \pm \nabla_{l} N_{s}^{b^{ \pm}}\right)\right) n_{\mathrm{b}_{j}} d S \\
& +\frac{1}{2} \int_{\Gamma_{\mathrm{CI}}^{s}}\left( \pm N^{a^{ \pm}}\right) \mathcal{C}_{\boldsymbol{u}}^{\mathrm{b} \pm}{ }_{i j k l} \nabla_{l} N^{b^{ \pm}} n_{\mathrm{b}_{j}} d S \\
& +\frac{1}{2} \int_{\Gamma_{\mathrm{UI}}^{s}}\left( \pm N^{a^{ \pm}}\right) \mathcal{C}_{\boldsymbol{u}}^{ \pm} \boldsymbol{u}_{i j k l} \nabla_{l} N^{b^{ \pm}} n_{\mathrm{b}_{j}} d S \\
& +\int_{\Gamma_{\mathrm{UI}}^{s}}\left( \pm N^{a^{ \pm}}\right) n_{\mathrm{b} j}\left\langle\frac{\beta_{s}}{h_{s}} \mathcal{H}_{i j k l}\right\rangle\left( \pm N^{b^{ \pm}}\right) n_{\mathrm{b} l} d S \\
& +\frac{1}{2} \int_{\Gamma_{\mathrm{UI}}^{s}}\left( \pm N^{b^{ \pm}}\right) \mathcal{H}_{i k j l}^{ \pm} \nabla_{j} N^{a^{ \pm}} n_{\mathrm{bl}} d S \\
& K_{\boldsymbol{u} \tilde{e} I_{i}}^{s a^{ \pm} b^{ \pm}}=\frac{\partial f_{\boldsymbol{u} I_{i}}^{a^{ \pm}}}{\partial \tilde{e}^{b^{ \pm}}}=\int_{\Gamma_{\mathrm{UI}}^{s}}\left( \pm N^{a^{ \pm}}\right)\left\langle\frac{\partial \sigma_{i j}}{\partial \tilde{e}^{b}}\right\rangle n_{\mathrm{b}_{j}} d S \\
& =\frac{1}{2} \int_{\Gamma_{\mathrm{UI}}^{s}}\left( \pm N^{a^{ \pm}}\right) \mathcal{C}_{\boldsymbol{u} \tilde{e}_{i j}}^{ \pm} N^{b^{ \pm}} n_{\mathrm{b} j} d S ;
\end{aligned}
$$

- Internal non-local force stiffness matrices $K_{\tilde{e} u \operatorname{int}_{i}}^{e b}$ and $K_{\tilde{e} e \tilde{i n t}}^{e}{ }^{a b}$ :

$$
\begin{aligned}
K_{\tilde{e} \boldsymbol{u} \text { int }{ }_{k}}^{e}{ }^{a b} & =\frac{\partial f_{\tilde{e} i n t}^{e}{ }^{a}}{\partial u_{k}^{b}}=0, \\
K_{\tilde{e} \tilde{e} \text { int }}^{e}{ }^{a b} & =\frac{\partial f_{\tilde{e} i n t}^{e}{ }^{a}}{\partial \tilde{e}^{b}}=\int_{B^{e}}\left(N^{a} N^{b}+l_{\mathrm{c}}^{2} \nabla_{i} N^{a} \nabla_{i} N^{b}\right) d V ;
\end{aligned}
$$


- Internal local force stiffness matrices $K_{e u \text { int }_{i}}{ }^{a b}$ and $K_{e \tilde{e} \text { int }}^{e a b}$ :

$$
\begin{aligned}
K_{\text {eu int }}^{e}{ }_{k}^{a b} & =\frac{\partial f_{\text {eint }}^{e}{ }^{a}}{\partial u_{k}^{b}}=\int_{B^{e}} N^{a} \frac{\partial e}{\partial u_{k}^{b}} d V=\int_{B^{e}} N^{a} \mathcal{C}_{e u_{k i}} \nabla_{i} N^{b} d V \\
K_{\text {eẽ int }}^{e}{ }^{a b} & =\frac{\partial f_{e i n t}^{e}}{\partial \tilde{e}^{b}}=0
\end{aligned}
$$

- Interface non-local force stiffness matrices $K_{\tilde{e} u \mathrm{I}_{i}}^{s} a^{a^{ \pm} b^{ \pm}}$and $K_{\tilde{e} e \tilde{I}}^{s} a^{a^{ \pm} b^{ \pm}}$:

$$
\begin{aligned}
K_{\tilde{e} u \mathrm{I}_{k}}^{s} a^{ \pm} b^{ \pm}= & \frac{\partial f_{\tilde{e} I}^{s} a^{ \pm}}{\partial u_{k}^{b^{ \pm}}}=0 \\
K_{\tilde{e} \tilde{e} \mathrm{I}}^{s} a^{ \pm} b^{ \pm} & =\frac{\partial f_{\tilde{e} I}^{s} a^{ \pm}}{\partial \tilde{e}^{b^{ \pm}}}=\frac{1}{2} \int_{\Gamma_{\mathrm{U}}^{s}}\left( \pm N^{a^{ \pm}}\right) l_{\mathrm{c}}^{ \pm 2} \nabla_{i} N^{b^{ \pm}} n_{i}^{-} d S \\
& +\int_{\Gamma_{\mathrm{UI}}^{s}}\left( \pm N^{a^{ \pm}}\right)\left\langle\frac{\beta_{s}}{h_{s}} l_{\mathrm{c}}^{2}\right\rangle\left( \pm N^{b^{ \pm}}\right) d S+\frac{1}{2} \int_{\Gamma_{\mathrm{UI}}^{s}}\left( \pm N^{b^{ \pm}}\right) l_{\mathrm{c}}^{ \pm 2} \nabla_{i} N^{a^{ \pm}} n_{\mathrm{bi}} d S .
\end{aligned}
$$

\section{REFERENCES}

1. Lemaitre J. Coupled elasto-plasticity and damage constitutive equations. Computer Methods in Applied Mechanics and Engineering Sep 1985; 51(13):31-49, doi:10.1016/0045-7825(85)90026-X. URL http: / / www . sciencedirect.com/science/article/pii/004578258590026x.

2. Lemaitre J, Chaboche JL, Benallal A, Desmorat R. Mcanique des matriaux solides - 3me dition. Dunod, 2009.

3. Peerlings RHJ, Geers MGD, de Borst R, Brekelmans WAM. A critical comparison of nonlocal and gradientenhanced softening continua. International Journal of Solids and Structures Nov 2001; 38(4445):7723-7746, doi: 10.1016/S0020-7683(01)00087-7. URL http://www. sciencedirect.com/science/article/pii/ S0020768301000877.

4. Peerlings RHJ, de Borst R, Brekelmans WAM, Geers MGD. Localisation issues in local and nonlocal continuum approaches to fracture. European Journal of Mechanics - A/Solids Jan 2002; 21(2):175-189, doi: 10.1016/S0997-7538(02)01211-1. URL http://www.sciencedirect.com/science/article/pii/ S0997753802012111.

5. Bazant ZP, Belytschko TB, Chang T. Continuum Theory for StrainSoftening. Journal of Engineering Mechanics Dec 1984; 110(12):1666-1692, doi:10.1061/(ASCE)0733-9399(1984)110:12(1666). URL http:// ascelibrary.org/doi/10.1061/\%28ASCE290733-9399\%281984\%29110\%3A12\%281666\%29.

6. PijaudierCabot G, Bazant ZP. Nonlocal Damage Theory. Journal of Engineering Mechanics Oct 1987; 113(10):1512-1533, doi:10.1061/(ASCE)0733-9399(1987)113:10(1512). URL http: / / ascelibrary • org/ doi/10.1061/\%28ASCE290733-9399\%281987\%291133A10\%281512\%29.

7. Zbib HM, Aifantis EC. On the gradient-dependent theory of plasticity and shear banding. Acta Mechanica Mar 1992; 92(1-4):209-225, doi:10.1007/BF01174177. URL http://link.springer.com/article/ $10.1007 / \mathrm{BF} 01174177$.

8. Peerlings RHJ, De Borst R, Brekelmans WaM, De Vree JHP. Gradient Enhanced Damage for QuasiBrittle Materials. International Journal for Numerical Methods in Engineering Oct 1996; 39(19):33913403, doi:10.1002/(SICI)1097-0207(19961015)39:19/3391::AID-NME7〉3.0.CO;2-D. URL http: //onlinelibrary.wiley.com/doi/10.1002/ (SICI) 1097-0207 (19961015) 39:19<3391: : AID-NME 7>3.0.CO;2-D/abstract.

9. Geers MGD, de Borst R, Brekelmans WAM, Peerlings RHJ. Strain-based transient-gradient damage model for failure analyses. Computer Methods in Applied Mechanics and Engineering Jul 1998; 160(1):133-153, doi: 10.1016/S0045-7825(98)80011-X. URL http://www.sciencedirect.com/science/article/pii/ S $004578259880011 X$. 
10. Peerlings RHJ, de Borst R, Brekelmans WaM, Geers MGD. Gradient-enhanced damage modelling of concrete fracture. Mechanics of Cohesive-frictional Materials Oct 1998; 3(4):323-342, doi:10.1002/(SICI) 1099-1484(1998100)3:4〈323::AID-CFM51〉3.0.CO;2-Z. URL http://onlinelibrary.wiley.com/ doi/10.1002/(SICI) 1099-1484 (1998100) 3:4<323: :AID-CFM51>3.0.CO;2-Z/abstract.

11. Miehe C, Welschinger F, Hofacker M. Thermodynamically consistent phase-field models of fracture: Variational principles and multi-field FE implementations. International Journal for Numerical Methods in Engineering Sep 2010; 83(10):1273-1311, doi:10.1002/nme.2861. URL http://onlinelibrary.wiley.com/doi/10. $1002 /$ nme.2861/abstract.

12. de Borst R, Verhoosel CV. Gradient damage vs phase-field approaches for fracture: Similarities and differences. Computer Methods in Applied Mechanics and Engineering 2016; 312:78 - 94, doi:http:// dx.doi.org/10.1016/j.cma.2016.05.015. URL http://www.sciencedirect.com/science/article/ pii/S0045782516303796, phase Field Approaches to Fracture.

13. Steinke C, Zreid I, Kaliske M. On the relation between phase-field crack approximation and gradient damage modelling. Computational Mechanics 2017; 59(5):717-735, doi:10.1007/s00466-016-1369-9. URL http:// dx.doi.org/10.1007/s00466-016-1369-9.

14. Dugdale DS. Yielding of steel sheets containing slits. Journal of the Mechanics and Physics of Solids May 1960; 8(2):100-104, doi:10.1016/0022-5096(60)90013-2. URL http://www. sciencedirect.com/science/ article/pii/0022509660900132.

15. Barenblatt GI. The Mathematical Theory of Equilibrium Cracks in Brittle Fracture. Advances in Applied Mechanics, vol. 7, Dryden HL, Kuerti GvdDH, Howarth L, von Karman T (eds.). Elsevier, 1962; 55-129. URL http: //www.sciencedirect.com/science/article/pii/s0065215608701212, dOI: 10.1016/S00652156(08)70121-2.

16. Moes N, Dolbow J, Belytschko T. A finite element method for crack growth without remeshing. International Journal for Numerical Methods in Engineering Sep 1999; 46(1):131-150, doi:10.1002/(SICI)1097-0207(19990910)46: 1〈131::AID-NME726〉3.0.CO;2-J. URL http://onlinelibrary.wiley.com/doi/10.1002/ (SICI) 1097-0207 (19990910) 46:1<131: :AID-NME726>3.0.CO;2-J/abstract.

17. Moes N, Belytschko T. Extended finite element method for cohesive crack growth. Engineering Fracture Mechanics May 2002; 69(7):813-833, doi:10.1016/S0013-7944(01)00128-X. URL http://www. sciencedirect. $\mathrm{com} / \mathrm{science/article/pii/s001379440100128 \textrm {x } .}$

18. Armero F, Linder C. Numerical simulation of dynamic fracture using finite elements with embedded discontinuities. International Journal of Fracture Dec 2009; 160(2):119, doi:10.1007/s10704-009-9413-9. URL http: / / link . springer.com/article/10.1007/s10704-009-9413-9.

19. Hillerborg A, Modeer M, Petersson PE. Analysis of crack formation and crack growth in concrete by means of fracture mechanics and finite elements. Cement and Concrete Research Nov 1976; 6(6):773-781, doi: 10.1016/0008-8846(76)90007-7. URL http://www.sciencedirect.com/science/article/pii/ 0008884676900077 .

20. Camacho GT, Ortiz M. Computational modelling of impact damage in brittle materials. International Journal of Solids and Structures Aug 1996; 33(20):2899-2938, doi:10.1016/0020-7683(95)00255-3. URL http: //www . sciencedirect.com/science/article/pii/0020768395002553.

21. Pandolfi A, Guduru PR, Ortiz M, Rosakis AJ. Three dimensional cohesive-element analysis and experiments of dynamic fracture in C300 steel. International Journal of Solids and Structures Jul 2000; 37(27):3733-3760, doi: 10.1016/S0020-7683(99)00155-9. URL http://www. sciencedirect.com/science/article/pii/ S 0020768399001559 .

22. Needleman A. A Continuum Model for Void Nucleation by Inclusion Debonding. Journal of Applied Mechanics Sep 1987; 54(3):525-531, doi:10.1115/1.3173064. URL http: / / dx. doi .org/10.1115/1.3173064.

23. Tvergaard V. Effect of fibre debonding in a whisker-reinforced metal. Materials Science and Engineering: A Jun 1990; 125(2):203-213, doi:10.1016/0921-5093(90)90170-8. URL http: / / www . sciencedirect. com/ science/article/pii/0921509390901708.

24. Xu XP, Needleman A. Numerical simulations of fast crack growth in brittle solids. Journal of the Mechanics and Physics of Solids Sep 1994; 42(9):1397-1434, doi:10.1016/0022-5096(94)90003-5. URL http: //www . sciencedirect.com/science/article/pii/0022509694900035.

25. Mergheim J, Kuhl E, Steinmann P. A hybrid discontinuous Galerkin/interface method for the computational modelling of failure. Communications in Numerical Methods in Engineering Jul 2004; 20(7):511-519, doi: 10.1002/cnm.689. URL http://onlinelibrary.wiley.com/doi/10.1002/cnm.689/abstract.

26. Noels L, Radovitzky R. An explicit discontinuous Galerkin method for non-linear solid dynamics: Formulation, parallel implementation and scalability properties. International Journal for Numerical Methods in Engineering May 2008; 74(9):1393-1420, doi:10.1002/nme.2213. URL http://onlinelibrary.wiley.com/doi/ 
$10.1002 / \mathrm{nme} .2213 /$ abstract.

27. Radovitzky R, Seagraves A, Tupek M, Noels L. A scalable 3d fracture and fragmentation algorithm based on a hybrid, discontinuous Galerkin, cohesive element method. Computer Methods in Applied Mechanics and Engineering Jan 2011; 200(14):326-344, doi:10.1016/j.cma.2010.08.014. URL http://www . sciencedirect.com/science/article/pii/s0045782510002471.

28. Prechtel M, Leugering G, Steinmann P, Stingl M. Towards optimization of crack resistance of composite materials by adjustment of fiber shapes. Engineering Fracture Mechanics Apr 2011; 78(6):944-960, doi:10. 1016/j.engfracmech.2011.01.007. URL http://www.sciencedirect.com/science/article/pii/ S0013794411000117.

29. Becker G, Noels L. A full-discontinuous Galerkin formulation of nonlinear KirchhoffLove shells: elasto-plastic finite deformations, parallel computation, and fracture applications. International Journal for Numerical Methods in Engineering Jan 2013; 93(1):80-117, doi:10.1002/nme.4381. URL http: / / onlinelibrary • wiley • com/ doi/10.1002/nme.4381/abstract.

30. Wu L, Tjahjanto D, Becker G, Makradi A, Jerusalem A, Noels L. A micromeso-model of intra-laminar fracture in fiber-reinforced composites based on a discontinuous Galerkin/cohesive zone method. Engineering Fracture Mechanics May 2013; 104:162-183, doi:10.1016/j.engfracmech.2013.03.018. URL http://www . sciencedirect.com/science/article/pii/s0013794413001252.

31. Nguyen VP. Discontinuous Galerkin/extrinsic cohesive zone modeling: Implementation caveats and applications in computational fracture mechanics. Engineering Fracture Mechanics Sep 2014; 128:37-68, doi: 10.1016/j.engfracmech.2014.07.003. URL https://www.sciencedirect.com/science/article/ pii/S0013794414002136.

32. Hansbo P, Salomonsson K. A discontinuous galerkin method for cohesive zone modelling. Finite Elements in Analysis and Design 2015; 102103:1 - 6, doi:http://dx.doi.org/10.1016/j.finel.2015.04.008. URL http: //www . sciencedirect.com/science/article/pii/s0168874X15000657.

33. Mediavilla J, Peerlings RHJ, Geers MGD. Discrete crack modelling of ductile fracture driven by non-local softening plasticity. International Journal for Numerical Methods in Engineering Apr 2006; 66(4):661-688, doi:10.1002/ nme.1572. URL http://onlinelibrary.wiley.com/doi/10.1002/nme.1572/abstract.

34. Moes N, Stolz C, Bernard PE, Chevaugeon N. A level set based model for damage growth: The thick level set approach. International Journal for Numerical Methods in Engineering Apr 2011; 86(3):358-380, doi:10.1002/ nme.3069. URL http://onlinelibrary.wiley.com/doi/10.1002/nme.3069/abstract.

35. Wu L, Becker G, Noels L. Elastic damage to crack transition in a coupled non-local implicit discontinuous Galerkin/extrinsic cohesive law framework. Computer Methods in Applied Mechanics and Engineering Sep 2014; 279:379-409, doi:10.1016/j.cma.2014.06.031. URL http: / / www. sciencedirect. com/science/ article/pii/s0045782514002175.

36. Mazars J, Pijaudier-Cabot G. From damage to fracture mechanics and conversely: A combined approach. International Journal of Solids and Structures Aug 1996; 33(20):3327-3342, doi:10.1016/0020-7683(96)00015-7. URL http://www.sciencedirect.com/science/article/pii/0020768396000157.

37. Dufour F, Pijaudier-Cabot G, Choinska M, Huerta A. Extraction of a crack opening from a continuous approach using regularized damage models. Computers \& Concrete 2008; 5(4):375-388. URL https: //hal. archives-ouvertes. fr/hal-00333852.

38. Cazes F, Coret M, Combescure A, Gravouil A. A thermodynamic method for the construction of a cohesive law from a nonlocal damage model. International Journal of Solids and Structures Mar 2009; 46(6):1476-1490, doi:10.1016/j.ijsolstr.2008.11.019. URL http://www. sciencedirect.com/science/article/pii/ S0020768308004903.

39. Cuvilliez S, Feyel F, Lorentz E, Michel-Ponnelle S. A finite element approach coupling a continuous gradient damage model and a cohesive zone model within the framework of quasi-brittle failure. Computer Methods in Applied Mechanics and Engineering Sep 2012; 237240:244-259, doi:10.1016/j.cma.2012.04.019. URL http: //www.sciencedirect.com/science/article/pii/s0045782512001466.

40. Wang Y, Waisman H. From diffuse damage to sharp cohesive cracks: A coupled XFEM framework for failure analysis of quasi-brittle materials. Computer Methods in Applied Mechanics and Engineering 2016; 299:57-89, doi:10.1016/j.cma.2015.10.019. URL https://www.sciencedirect.com/science/article/pii/ S 0045782515003485.

41. Wu CT, Ma N, Takada K, Okada H. A meshfree continuousdiscontinuous approach for the ductile fracture modeling in explicit dynamics analysis. Computational Mechanics 2016; 58(3):391-409, doi:10.1007/s00466-016-1299-6. URL http: / / link.springer.com/article/10.1007/s00466-016-1299-6.

42. Remmers JJC, Borst Rd, Verhoosel CV, Needleman A. The cohesive band model: a cohesive surface formulation with stress triaxiality. International Journal of Fracture Jun 2013; 181(2):177-188, 
doi:10.1007/s10704-013-9834-3. s10704-013-9834-3.

43. Tvergaard V, Hutchinson JW. The relation between crack growth resistance and fracture process parameters in elastic-plastic solids. Journal of the Mechanics and Physics of Solids Aug 1992; 40(6):1377-1397, doi: 10.1016/0022-5096(92)90020-3. URL http://www.sciencedirect.com/science/article/pii/ 0022509692900203.

44. Nahshon K, Hutchinson JW. Modification of the Gurson Model for shear failure. European Journal of Mechanics - A/Solids Jan 2008; 27(1):1-17, doi:10.1016/j.euromechsol.2007.08.002. URL http://www. sciencedirect.com/science/article/pii/s0997753807000721.

45. Faleskog J, Barsoum I. Tensiontorsion fracture experimentsPart I: Experiments and a procedure to evaluate the equivalent plastic strain. International Journal of Solids and Structures Dec 2013; 50(2526):4241-4257, doi:10.1016/j.ijsolstr.2013.08.029. URL http://www.sciencedirect.com/science/article/pii/ S 0020768313003454 .

46. Xue Z, Faleskog J, Hutchinson JW. Tensiontorsion fracture experiments Part II: Simulations with the extended Gurson model and a ductile fracture criterion based on plastic strain. International Journal of Solids and Structures Dec 2013; 50(2526):4258-4269, doi:10.1016/j.ijsolstr.2013.08.028. URL http://www.sciencedirect. $\mathrm{com} / \mathrm{science/article/pii/s0020768313003442.}$

47. Siegmund T, Brocks W. A numerical study on the correlation between the work of separation and the dissipation rate in ductile fracture. Engineering Fracture Mechanics Sep 2000; 67(2):139-154, doi: 10.1016/S0013-7944(00)00054-0. URL http://www. sciencedirect.com/science/article/pii/ S0013794400000540.

48. Tvergaard V, Hutchinson JW. Effect of strain-dependent cohesive zone model on predictions of crack growth resistance. International Journal of Solids and Structures Aug 1996; 33(20):3297-3308, doi: 10.1016/0020-7683(95)00261-8. URL http://www.sciencedirect.com/science/article/pii/ 0020768395002618.

49. Varias AG. Constraint effects during stable transient crack growth. Computational Mechanics May 1998; 21(45):316-329, doi:10.1007/s004660050308. URL https://link.springer.com/article/10.1007/ s004660050308.

50. Gurson AL. Continuum Theory of Ductile Rupture by Void Nucleation and Growth: Part IYield Criteria and Flow Rules for Porous Ductile Media. Journal of Engineering Materials and Technology Jan 1977; 99(1):2-15, doi: 10.1115/1.3443401. URL http://dx. doi.org/10.1115/1.3443401.

51. Tvergaard V. Predictions of mixed mode interface crack growth using a cohesive zone model for ductile fracture. Journal of the Mechanics and Physics of Solids Apr 2004; 52(4):925-940, doi:10.1016/S0022-5096(03)00115-7. URL http: / / www. sciencedirect.com/science/article/pii/s0022509603001157.

52. Huespe AE, Needleman A, Oliver J, Sanchez PJ. A finite thickness band method for ductile fracture analysis. International Journal of Plasticity Dec 2009; 25(12):2349-2365, doi:10.1016/j.ijplas.2009.03.005. URL http: //www.sciencedirect.com/science/article/pii/s0749641909000461.

53. Huespe AE, Needleman A, Oliver J, Sanchez PJ. A finite strain, finite band method for modeling ductile fracture. International Journal of Plasticity Jan 2012; 28(1):53-69, doi:10.1016/j.ijplas.2011.05.010. URL http: / / www . sciencedirect.com/science/article/pii/s0749641911000866.

54. Esmaeili A, Javili A, Steinmann P. Coherent energetic interfaces accounting for in-plane degradation. International Journal of Fracture 2016; 202(2):135-165, doi:10.1007/s10704-016-0160-4. URL http: / / ink . springer. com/article/10.1007/s10704-016-0160-4.

55. Esmaeili A, Javili A, Steinmann P. A thermo-mechanical cohesive zone model accounting for mechanically energetic kapitza interfaces. International Journal of Solids and Structures 2017; 9293:29-44, doi: 10.1016/j.ijsolstr.2016.04.035. URL https://www.sciencedirect.com/science/article/pii/ S 0020768316300592 .

56. Esmaeili A, Steinmann P, Javili A. Non-coherent energetic interfaces accounting for degradation. Computational Mechanics Mar 2017; 59(3):361-383, doi:10.1007/s00466-016-1342-7. URL https://Iink.springer. com/article/10.1007/s00466-016-1342-7.

57. Coenen EWC, Kouznetsova VG, Bosco E, Geers MGD. A multi-scale approach to bridge microscale damage and macroscale failure: a nested computational homogenization-localization framework. Int J Fract Nov 2012; 178(12):157-178, doi:10.1007/s10704-012-9765-4. URL http:// link. springer.com/article/10.1007/ s10704-012-9765-4.

58. Bosco E, Kouznetsova VG, Coenen EWC, Geers MGD, Salvadori A. A multiscale framework for localizing microstructures towards the onset of macroscopic discontinuity. Comput Mech Aug 2014; 54(2):299-319, doi:10.1007/s00466-014-0986-4. URL http://link.springer.com/article/10. 
1007/s00466-014-0986-4.

59. Miehe C, Hofacker M, Welschinger F. A phase field model for rate-independent crack propagation: Robust algorithmic implementation based on operator splits. Computer Methods in Applied Mechanics and Engineering 2010; 199(45):2765 - 2778, doi:http://dx.doi.org/10.1016/j.cma.2010.04.011. URL http://www . sciencedirect.com/science/article/pii/s0045782510001283.

60. Geers MM. Experimental analysis and computational modelling of damage and fracture. $\mathrm{PhD}$ Thesis, University of Technology, Eindhoven (Netherlands) 1997. URL https://doi .org/10.6100/IR477352.

61. Riks E. An incremental approach to the solution of snapping and buckling problems. International Journal of Solids and Structures 1979; 15(7):529 - 551, doi:http://dx.doi.org/10.1016/0020-7683(79)90081-7. URL http: //www.sciencedirect.com/science/article/pii/0020768379900817.

62. Borst RD, Crisfield MA, Remmers JJC, Verhoosel CV. Nonlinear Finite Element Analysis of Solids and Structures. John Wiley \& Sons, 2012.

63. Dufour F, Pijaudier-Cabot G, Choinska M, Huerta A. Extraction of a crack opening from a continuous approach using regularized damage models. Computers \& Concrete 2008; 5(4):375-388. URL https:// hal. archives-ouvertes.fr/hal-00333852.

64. Noels L, Radovitzky R. A general discontinuous Galerkin method for finite hyperelasticity. Formulation and numerical applications. International Journal for Numerical Methods in Engineering Oct 2006; 68(1):6497, doi:10.1002/nme.1699. URL http://onlinelibrary.wiley.com/doi/10.1002/nme.1699/ abstract.

65. Molinari JF, Gazonas G, Raghupathy R, Rusinek A, Zhou F. The cohesive element approach to dynamic fragmentation: the question of energy convergence. International Journal for Numerical Methods in Engineering Jan 2007; 69(3):484-503, doi:10.1002/nme.1777. URL http://onlinelibrary.wiley.com/doi/10. 1002 /nme.1777/abstract.

66. Geuzaine C, Remacle JF. Gmsh: A 3-D finite element mesh generator with built-in pre- and post-processing facilities. International Journal for Numerical Methods in Engineering Sep 2009; 79(11):1309-1331, doi:10.1002/ nme.2579. URL http: //onlinelibrary.wiley.com/doi/10.1002/nme.2579/abstract.

67. Zhou F, Molinari JF. Stochastic fracture of ceramics under dynamic tensile loading. International Journal of Solids and Structures Nov 2004; 41(2223):6573-6596, doi:10.1016/j.ijsolstr.2004.05.029. URL http://www . sciencedirect.com/science/article/pii/s0020768304002562.

68. Hulbert GM, Chung J. Explicit time integration algorithms for structural dynamics with optimal numerical dissipation. Computer Methods in Applied Mechanics and Engineering Oct 1996; 137(2):175-188, doi: 10.1016/S0045-7825(96)01036-5. URL http://www. sciencedirect.com/science/article/pii/ S 0045782596010365 . 